\title{
Fibrados vetoriais sobre "spherical space forms" tridimensionais $^{1}$
}

\author{
Esdras Teixeira Costa
}

Orientador: Prof. Dr. Mauro Flávio Spreafico

Tese apresentada ao Instituto de Ciências Matemáticas e de Computação - ICMC-USP, como parte dos requisitos para obtenção do título de Doutor em Ciências - Matemática.

USP - São Carlos

Fevereiro de 2006

(Versão revisada)

Data da defesa: $31 / 03 / 2006$

Visto do orientador:

${ }^{1}$ Trabalho realizado com o auxílio financeiro do $\mathrm{CNPq}$ 
Aos meus pais

Joaquim Teixeira da Silva

e

Maria de Lourdes Costa Silva

e à minha irmã

Lia Teixeira Costa 


\section{Agradecimentos}

Ao meu orientador Mauro F. Spreafico por toda sua paciência e disposição, pelo empenho descomunal com que trabalha e pela infindável boa vontade para comigo. Ao Dide, cuja ajuda (seja ela em matemática ou não) foi onipresente e onipotente durante todo meu doutorado e mestrado. Ao Conde, que sempre me incentivou e me ajudou no que pode, inclusive tornando possível meu ingresso no programa de doutorado. Estarei em débito com vocês pelo resto da vida...

A todos os amigos que fiz em São Carlos... não citarei nomes para não correr o risco de esquecer ninguém. Gostaria de citar um por um mas infelizmente, como diria Fermat, o espaço que me resta nesta folha não é suficiente. Vocês sabem quem são.

A todos os professores e funcionários da Universidade de São Paulo.

Por fim, citando Mr. Bean: aos garçons!!! 


\section{Resumo}

Neste trabalho consideramos o problema de enumerar $G$-fibrados sobre variedades de dimensão baixa (menor ou igual a 3), em particular fibrados vetoriais sobre as "spherical space forms" tridimensionais. É dada uma resposta completa para estas questões e na seção 5.1 são colocadas tabelas que explicitam os possíveis fibrados vetoriais sobre as "spherical space forms". Este tipo de problema é recorrente em topologia algébrica e por motivos dados pela teoria de homotopia, é preciso calcular certos invariantes algébricos com sistemas de coeficientes locais, o que torna o problema mais interessante. Mostramos ainda que sobre condições consideravelmente abrangentes no grupo estrutural $G$, os $G$-fibrados sobre variedades de dimensão menor ou igual a três podem ser enumerados de maneira efetiva. 


\section{Abstract}

In this work we consider the problem of enumerating $G$-bundles over low dimensional manifolds (dimension $\leq 3$ ) and in particular vector bundles over the three dimensional "spherical space forms". We give a complete answer to these questions and in section 5.1 we give tables for the possible vector bundles over the "spherical space forms". We deal with the problem of enumerating vector bundles over a class of manifolds. This is a long standing classical problem in algebraic topology, and because of homotopy theoretical reasons, it implies calculations of algebraic invariants with local system of coefficients, and thus becomes a cumbersome target away from the trivial occurrences. Although, we show that, under reasonably wide assumptions on the structure group $G, G$-bundles over low (lower or equal to three) dimensional manifolds can be counted effectively. 


\section{Sumário}

$\begin{array}{lr}\text { Introdução } & 1\end{array}$

1 Classificação de fibrados $\quad 3$

1.0.1 Equivalência de fibrados . . . . . . . . . . . . . 7

1.0.2 Classificação de $G$-fibrados principais . . . . . . . . . . . . 8

1.0.3 Classes características e soma de Whitney . . . . . . . . . 10

2 Coeficientes locais e resoluções $\quad 15$

2.1 Sheaves . . . . . . . . . . . . . . . . . . . 15

2.1.1 Sistemas de coeficientes locais. . . . . . . . . . . . 16

2.2 Homologia com coeficientes locais. . . . . . . . . . . . . . . . 21

2.3 Resoluções . . . . . . . . . . . . . . . . . . . . . . . . . . . . . . . . . . . 28

3 Torres de Postnikov e seqüências espectrais 32

3.1 Seqüências espectrais . . . . . . . . . . . . . . . . . . 35

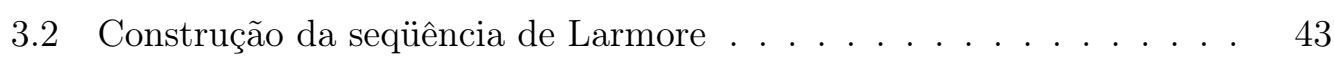

3.3 Exemplos . . . . . . . . . . . . . . . . . . . . . 50

4 Resultados gerais obtidos para classificação de fibrados $\quad 65$

$\begin{array}{lll}5 & \text { Fibrados sobre spherical space forms tridimensionais. } & 71\end{array}$

5.0 .1 Spherical space forms . . . . . . . . . . . . . . 71

5.0 .2 Cálculos . . . . . . . . . . . . . . . . . . . 74

5.1 Enumerando fibrados . . . . . . . . . . . . . . . . . . . 80

6 Apêndice $\quad 83$

6.1 O produto Cup . . . . . . . . . . . . . . . 83

6.1.1 A estrutura multiplicativa do anel $H^{*}(Q(2 n) ; R) \ldots \ldots . . . \quad 83$

6.2 Os quadrados de Steenrod . . . . . . . . . . . . . . 87 
6.3 Relações entre fibrados vetoriais reais e complexos . . . . . . . . . . 88

6.4 Levantamentos de aplicações em torres de Postnikov . . . . . . . . . 89

$6.4 .1 \quad \mathrm{O}$ caso de $O_{3} \ldots \ldots \ldots \ldots \ldots$

$\begin{array}{lr}\text { Referências Bibliográficas } & 94\end{array}$ 


\section{Introdução}

Este trabalho trata da classificação de fibrados sobre variedades tridimensionais denominadas "spherical space forms", que nada mais são do que quocientes da esfera $S^{3}$ obtidos a partir da ação de grupos descontínuos de isometrias que agem sem pontos fixos.

O problema de classificação de fibrados surgiu para nós durante o estudo do trabalho de Lawrence L. Larmore em [11] e posteriormente em [10], onde Larmore enumera fibrados vetoriais sobre espaços projetivos de dimensão menor ou igual a cinco. Em particular, o conteúdo de [11] serviu como principal referência para a maior parte do trabalho e nos guiou até a elaboração de dois teoremas sobre classificação de fibrados sobre variedades de dimensão baixa (menor ou igual a três).

A partir destes resultados e da classificação das "spherical space forms" encontradas nos trabalhos de Seifert e Therelfall em [20], onde os grupos descontínuos de isometrias que agem sem pontos fixos em $S^{n}$ foram listados, elaboramos as tabelas de classificação presentes na parte final desta tese.

A tese se divide em seis partes, sendo que na primeira delas tratamos da teoria geral de classificação de fibrados (cuja importância é óbvia) e ainda de classes características e somas de Whitney, elementos fundamentais para se distinguir os fibrados nas tabelas da seção 5.1 .

O capítulo seguinte trata de coeficientes locais e resoluções, ambos tópicos necessários para a realização dos cálculos dos grupos de homologia e cohomologia que acabam por enumerar os fibrados sobre as "spherical space forms", como veremos nos capítulos posteriores. A natureza puramente técnica de alguns resultados aqui apresentados fez com que omitíssemos a demonstração destes e concentrássemos a maior parte dos detalhes nos ítens mais relevantes para este trabalho.

A seguir temos um capítulo dedicado totalmente ao estudo das seqüências espectrais e das torres de Postnikov. Demos especial atenção à seqüência espectral desenvolvida pela primeira vez por L. L. Larmore em [11], uma vez que esta tem papel central no processo utilizado por nós para classificar fibrados. 
O próximo capítulo mostra os resultados gerais obtidos para fibrados sobre quaisquer variedades fechadas de dimensão menor ou igual a três, cujo grupo estrutural é um grupo de Lie conexo (veja o teorema 4.1) ou compacto (teorema 4.2). Tais teoremas são abrangentes o suficiente para cobrir os casos mais interessantes e importantes de fibrados cujo grupo estrutural é um grupo de Lie.

Depois destes resultados estamos em condição de classificar os fibrados vetoriais de dimensão menor ou igual a três sobre as "spherical space forms", o que é feito de maneira detalhada no quinto capítulo, culminando nas tabelas de classificação que resumem todos os cálculos aqui apresentados.

Por fim, no apêndice temos o material que foi confeccionado para a tese mas que por um motivo ou por outro, seja falta de ligação com os elementos principais de cada capítulo ou ainda para evitar "quebras" indesejadas no ritmo de leitura, não se adequaram a nenhum capítulo anterior. 


\section{Capítulo 1}

\section{Classificação de fibrados}

Neste primeiro capítulo faremos uma breve introdução à classificação de fibrados. A abordagem que daremos ao tema pede que tratemos primeiramente de propriedades algébricas e topológicas de grupos topológicos e sua ação em espaços topológicos. Faremos isso da forma mais geral possível. As principais referências aqui são os trabalhos de [9], [16], [21], onde podem ser encontradas as demonstrações de todas as proposições e teoremas não demonstrados nesta seção.

Definição 1.1. Dizemos que um espaço topológico $G$ é um grupo topológico se $G$ é um grupo abstrato (algébrico) munido de uma operação de multiplicação

$$
\begin{gathered}
G \times G \rightarrow G \\
(g, h)=g h
\end{gathered}
$$

que é contínua na topologia de $G$, assim como a aplicação que leva $g$ até $g^{-1}$.

Definição 1.2. Um grupo topológico $G$ age à direita em um espaço $X$ se existe uma aplicação $\varphi: X \times G \rightarrow X$, chamada de ação à direita, definida por $\varphi(x, g)=x g$ de tal forma que $\varphi(x, g h)=\varphi(\varphi(x, g), h)$ e ainda $\varphi(x, u)=x$, onde $u$ é a unidade de $G$.

Uma ação à esquerda é definida de maneira completamente análoga. Nas condições acima, o par $(X, \varphi)$ é chamado de $G$-espaço à direita e também dizemos que $G$ é um de grupo de transformações de $X$.

Definição 1.3. A ação $\varphi$ de um grupo $G$ em um espaço $X$ é chamada de efetiva se o kernel de $g \mapsto L_{g}$ é trivial, onde $L_{g}=x g, x \in X$. Mais ainda, $\varphi$ é dita livre se $x g=x$ implica $g=u$. 
Definição 1.4. Sejam $X$ e $Y$ G-espaços. Uma aplicação $f: X \rightarrow Y$ é chamada $G$-equivariante ou $G$-aplicação se $f(x g)=f(x) g$ para todo $x \in X$ e $g \in G$.

Uma $G$-aplicação $f: X \rightarrow Y$ induz uma aplicação quociente entre os espaços das órbitas que pode ser representada no seguinte diagrama:

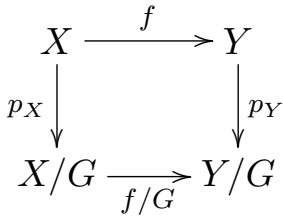

A categoria dos $G$-espaços e $G$-aplicações é denotada por $T o p_{G}$, e os isomorfismos nesta categoria são chamados de $G$-homeomorfismos.

Definição 1.5. Seja $\varphi: X \times G \rightarrow X$ uma ação livre. Considere $X^{*}=\{(x, x g) \mid x \in$ $X, g \in G\}$ como subespaço de $X \times X$ e defina a função translaçãoo $\tau_{X}$ do $G$-espaço $X$ por

$$
\begin{gathered}
\tau_{X}: X^{*} \rightarrow G, \\
\tau_{X}(x, x g)=g .
\end{gathered}
$$

Tal função satisfaz as seguintes propriedades:

- $\tau_{X}(x, x)=u_{G}$

- $\tau_{X}\left(x, x^{\prime}\right) \tau_{X}\left(x^{\prime}, x\right)=u_{G}$;

- $\tau_{X}\left(x, x^{\prime}\right) \tau_{X}\left(x^{\prime}, x^{\prime \prime}\right)=\tau_{X}\left(x, x^{\prime \prime}\right)$;

- $x \tau_{X}\left(x, x^{\prime}\right)=x^{\prime}$

Uma $G$-ação é chamada de principal se $\tau_{X}$ é uma aplicação contínua. Também dizemos que um $G$-espaço livre com uma ação principal é um $G$-espaço principal.

Definição 1.6. Um $G$-espaço $X$ é chamado localmente trivial se cada ponto $x G$ do espaço das órbitas $X / G$ tem uma vizinhança $V$ tal que $p^{-1}(V)$ é $G$-homeomorfa a $V \times G$, onde $p: X \rightarrow X / G$ é a projeção canônica.

Proposição 1. Seja $X$ um $G$-espaço livre e suponha que $X$ é localmente trivial. Então $\tau_{X}$ é contínua.

Definição 1.7. Se $p_{r_{1}}$ é a projeção no primeiro fator, um G-fibrado principal consiste de uma $G$-ação livre à direita $E \times G \mapsto E$ e uma aplicação sobrejetora $p: E \rightarrow B$ tais que: 
- $p(e g)=p(e) g$

- Para cada $b \in B$ existe uma vizinhança $V$ de $b$ em $B$ e um $G$-homeomorfismo $\varphi: p^{-1}(V) \rightarrow V \times G$ de tal maneira que o diagrama a seguir comuta:

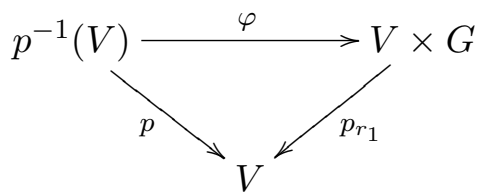

O espaço $E$ na definição acima é chamado de espaço total do fibrado, $B$ é chamado de espaço base e $\varphi$ é a trivialização sobre $V$.

Seja $p: E \rightarrow B$ um $G$-fibrado principal. Seja $F$ um espaço com uma $G$-ação efetiva à esquerda. Então podemos construir um novo fibrado a partir de $p$ como se segue: Façamos $G$ agir no produto $E \times F$ colocando $(x, y) g=\left(x g, g^{-1} y\right)$, definamos $E \times{ }_{G} F=(E \times R) / G \mathrm{e}$

$$
\begin{gathered}
p_{F}: E \times_{G} F \rightarrow B=E / G, \\
{[(x, y)] \mapsto p(x) .}
\end{gathered}
$$

Definição 1.8. A 5-upla $\left(E \times_{G} F, p_{F}, B, F, G\right)$ é chamado de fibrado com fibra $F$ e grupo $G$ sobre $B$ associado a $p$.

A definição acima para um $G$-fibrado principal é bastante global. O espaço total tem uma ação expressa por uma função definida globalmente, e a função de translação é bem definida em todo um espaço. Veremos agora uma definição equivalente de $G$ fibrado principal que toma o ponto de vista local, partindo da introdução de um conjunto de funções locais.

Definição 1.9. Um fibrado é uma 5-upla $\xi=(E, p, B, F, G)$ satisfazendo as seguintes propriedades:

1. E, $B, F$ são espaços topológicos, $p: E \rightarrow B$ é uma aplicação e $G$ é um grupo topológico agindo efetivamente à esquerda de $F$, o que é equivalente a dizer que $G \subseteq \operatorname{Homeo}(F)$;

2. B é coberto por uma coleção de abertos $\mathbb{U}=\left\{U_{i}\right\}_{i \in J}$ e para todo $i \in J$ existe um homeomorfismo $\varphi_{i}: U_{i} \times F \rightarrow p^{-1}\left(U_{i}\right)$ sobre $U_{i}$ de tal maneira que $p \varphi_{i}=p_{r_{1}}$;

3. Para cada $b \in U_{i j}=U_{i} \cap U_{j} \neq 0$ e todo $y \in F, \varphi_{i}^{-1} \varphi_{j}(b, y)=\left(b, g_{i j}(b) y\right)$ e $a$ função $g_{i j}: U_{i j} \rightarrow G$ é contínua. 
O espaço $E$ é o espaço total do fibrado $\xi, B$ é a base, $F$ é a fibra e $G$ é o grupo estrutural. Mais ainda, chamamos as aplicações $g_{i j}$ de funções de transição. Um fibrado com grupo estrutural $G$ é também chamado de $G$-fibrado. Se a fibra de um fibrado é um espaço vetorial, dizemos que este é um fibrado vetorial.

\section{Exemplo 1.1.}

- O fibrado produto sobre $B$ com fibra $F$ é $(B \times F, p, B, F, 0)$, onde p é a projeção no primeiro fator e 0 é o grupo trivial.

- O fibrado tangente sobre $S^{n}$, denotado por $T=\tau\left(S^{n}\right)=\left(T, p, S^{n}, \mathbb{R}^{n}, O_{n}(\mathbb{R})\right)$ e o fibrado normal por $N=\nu\left(S^{n}\right)=\left(N, q, S^{n}, \mathbb{R}^{n}, O_{n}(\mathbb{R})\right)$ são dois fibrados cujos espaços totais são definidos pela relação $(b, x) \in T$ se e somente se o produto interno $(b \mid x)=0$ e $(b, x) \in N$ se e somente se $x=k b$ para algum $k \in \mathbb{R}$. As fibras $p^{-1}(b) \subset T$ e $q^{-1}(b) \subset N$ são espaços vetoriais de dimensões $n$ e 1 , respectivamente. Um cross section de $\tau\left(S^{n}\right)$ é chamado de campo vetorial (tangente) em $S^{n}$ e um cross section de $\nu\left(S^{n}\right)$ é chamado de campo vetorial normal em $S^{n}$.

A variedade de Stiefel de $k$-frames (ortogonais) em $\mathbb{R}^{n}$, denotada por $V_{k}\left(\mathbb{R}^{n}\right)$, é o subespaço dos $\left(v_{1}, \ldots, v_{k}\right) \in\left(S^{n-1}\right)^{k}$ tais que $\left(v_{i} \mid v_{j}\right)=\delta_{i, j}$ (informalmente, a variedade de Stiefel é o subespaço das $k$-uplas de vetores ortonormais). Como $V_{k}\left(\mathbb{R}^{n}\right)$ é um subespaço fechado de um espaço compacto, é também um espaço compacto. A cada $k$-frame $\left(v_{1}, \ldots, v_{k}\right)$ é associado o subespaço $k$-dimensional $\left\langle v_{1}, \ldots v_{k}\right\rangle$ com base $v_{1}, \ldots, v_{k}$. A variedade Grassmaniana de $k$-subespaços de $\mathbb{R}^{n}$, denotada por $G_{k}\left(\mathbb{R}^{n}\right)$, é o conjunto dos subespaços $k$-dimensionais de $\mathbb{R}^{n}$ com a topologia quociente definida pela função $\left(v_{1}, \ldots, v_{k}\right) \rightarrow<v_{1}, \ldots, v_{k}>$ de $V_{k}\left(\mathbb{R}^{n}\right)$ sobrejetora em $G_{k}\left(\mathbb{R}^{n}\right)$. A variedade Grassmaniana também é um espaço compacto. Note que $V_{1}\left(\mathbb{R}^{n}\right)=S^{n-1}$ e $G_{1}\left(\mathbb{R}^{n}\right)=\mathbb{R} P^{n-1}$. Mais à frente usaremos o fato de que a Grassmaniana $G_{K}\left(F^{n}\right)$ é o espaço de classificação de uma grande classe de fibrados vetoriais.

Exemplo 1.2. A projeção $p_{k}^{n}: V_{k}\left(\mathbb{R}^{n}\right) \rightarrow G_{k}\left(\mathbb{R}^{n}\right)$ da variedade de Stiefel na grassmaniana determina um $O_{k}(\mathbb{R})$-fibrado principal

$$
\xi_{O_{k}(\mathbb{R})}=\left(V_{k}\left(\mathbb{R}^{n}\right), p_{k}^{n}, G_{k}\left(\mathbb{R}^{n}\right), O_{k}(\mathbb{R}), O_{k}(\mathbb{R})\right)
$$

chamado de fibrado universal real $k$-dimensional. O fibrado vetorial associado com fibra $\mathbb{R}^{k}$ é chamado de fibrado vetorial real k-dimensional canônico, e é denotado por $\gamma_{k}^{n}$. O espaço total deste fibrado pode ser visto como um subconjunto do produto $G_{k}\left(\mathbb{R}^{n}\right) \times \mathbb{R}^{n}$, a saber, o subconjunto dos elementos $(V, x)$ tais que $x \in V ; A$ projeção deste fibrado é a projeção no primeiro componente 
As funções de transição são muito importantes quando se trabalha com fibrados, e a seguinte formulação para tais apliações é de grande utilidade: para cada $b \in U_{i j}$, defina

$$
\begin{gathered}
\varphi_{i, b}:\{b\} \times F \rightarrow p^{-1}(b) \\
\varphi_{i, b}(y)=\varphi_{i}(b, y),
\end{gathered}
$$

assim, para cada $y \in F, g_{i j}(b) y=\varphi_{i, b}^{-1} \varphi_{j, b}(y)$.

As aplicações de transição possuem as seguintes propriedades:

- (FT1) $\forall b \in U_{i}, g_{i i}(b)=u$;

- $(\mathrm{FT} 2) \forall b \in U_{i j},\left(g_{i j}(b)\right)^{-1}=g_{j i}(b)$;

- $(\mathrm{FT} 3) \forall b \in U_{i j k}, g_{k i}(b)=g_{k j}(b) g_{j i}(b)$;

Proposição 2. Seja $G$ um grupo topológico que age efetivamente à esquerda em um espaço $F$; Seja $B$ um espaço com uma cobertura aberta $\left\{U_{i}\right\}_{i \in J}$ tal que para cada $i, j \in J$ para os quais $U_{i j} \neq \emptyset$ temos aplicações $g_{i j}: U_{i j} \rightarrow G$ que satisfazem as condições FT1, FT2 e FT3 acima. Então existe um fibrado $\xi=(E, p, B, F, G)$ com aplicações de transição $g_{i j}$.

Esta proposição nos permite construir um $G$-fibrado com fibra $G$ a partir de qualquer $G$-fibrado $\xi$ dado: basta ver que $G$ age nele mesmo por multiplicação. Tal $G$-fibrado com fibra $G$ é chamado de $G$-fibrado principal associado a $\xi$. É importante observar que demos aqui duas definições para $G$-fibrado principal e que as duas são equivalentes.

Devido à relativa simplicidade na categoria dos $G$-fibrados principais, daqui até o fim deste capítulo os resultados serão dados apenas em termos de fibrados principais. Tais resultados são "traduzidos" prontamente para os fibrados associados ao se utilizar a construção acima.

Os objetos na categoria $\operatorname{Princ}_{G}(B)$ são $G$-fibrados principais sobre o espaço $B$; um morfismo $f \in \operatorname{Princ}_{G}\left(\xi, \xi^{\prime}\right)$ é uma aplicação $G$-equivariante $f: E \rightarrow E^{\prime}$ que comuta com as projeções $p$ e $p^{\prime}$.

\subsubsection{Equivalência de fibrados}

Usando a definição global 1.7 é fácil introduzir a noção natural de equivalência de fibrados - os isomorfismos na categoria $\operatorname{Princ}_{G}(B)$. G-fibrados principais isomorfos são também chamados equivalentes, e temos aqui claramente uma relação de equivalência. A proposição a seguir também serve como uma definição dada a partir das funções de transição: 
Proposição 3. Sejam $\xi$ e $\xi^{\prime}$ dois G-fibrados com a mesma base B e fibra F. Suponha ainda que estes dois fibrados são localmente triviais sobre a mesma cobertura aberta de $B$. Então $\xi$ e $\xi^{\prime}$ são equivalentes se e somente se para todo $i \in J$ existe uma aplicação $\rho_{i}: U_{i} \rightarrow G$ tal que $g_{i j}^{\prime}(b)=\left(\rho_{j}(b)\right)^{-1} g_{i j}(b) \rho_{i}(b)$, para todo $b \in U_{i j}$.

É claro que nestas condições, dois $G$-fibrados são equivalentes (ou isomorfos) se e somente se seus $G$-fibrados principais associados são equivavalentes. Um fibrado é dito trivial se é equivalente ao fibrado produto.

Definição 1.10. Seja $\xi=(E, p, B . F, G)$ um fibrado, e $A$ um subconjunto de $B$. Então a restrição de $\xi$ a A, denotada por $\xi_{A}$, é o fibrado $\left(E^{\prime}, p^{\prime}, A, F, G\right)$, onde $E^{\prime}=p^{-1}(A)$ e $p^{\prime}=\left.p\right|_{E^{\prime}}$.

Definição 1.11. Seja $\xi=(E, p, B, F, G)$ um fibrado, e seja $f: B_{1} \rightarrow B$ uma aplicação. O fibrado induzido de $\xi$ sobre $f$, denotado por $f^{*}(\xi)$ ou $f^{-1}(\xi)$, tem espaço base $B_{1}$, como espaço total $E_{1}$ o subespaço de todos os pares $\left(b_{1}, x\right) \in B_{1} \times E$ com $f\left(b_{1}\right)=p(x)$, e como projeção $p_{1}$ a aplicação $\left(b_{1}, x\right) \mapsto b_{1}$. A fibra e o grupo estrutural são os mesmos de $\xi$. (O fibrado induzido não é nada mais que o pull-back do fibrado original por $f$.)

Se $f^{*}(\xi)$ é o fibrado induzido de $\xi$ sobre $f: B_{1} \rightarrow B$, então $f_{\xi}: E\left(f^{*}(\xi)\right) \rightarrow E(\xi)$, definida por $f_{\xi}\left(b_{1}, x\right)=x$ juntamente com $f$ definem um morfismo $\left(f_{\xi}, f\right): f^{*}(\xi) \rightarrow$ $\xi$, que é chamado de morfismo canônico de um fibrado induzido. O diagrama a seguir descreve a situação:

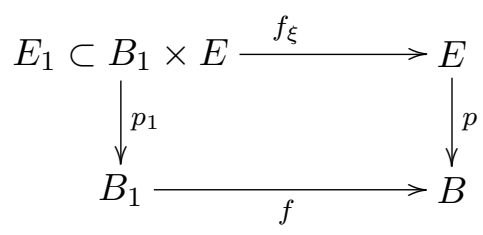

Como exemplo de restrição de fibrado temos o fibrado vetorial canônico $k$-dimensional sobre as grassmanianas, $\left.\gamma_{k}^{n+m}\right|_{G_{k}\left(\mathbb{R}^{n}\right)}=\gamma_{k}^{n}$. Se temos ainda um fibrado $\xi$ sobre $B$ e $A$ é um subespaço de $B$ com aplicação inclusão $j: A \rightarrow B$, então $j^{*}(\xi)$ é $A$-isomorfo a $\left.\xi\right|_{A}$ devido ao $A$-isomorfismo $u:\left.\xi\right|_{A} \rightarrow j^{*}(\xi)$ dado por $u(x)=(p(x), x)$.

\subsubsection{Classificação de $G$-fibrados principais}

Nosso objetivo nesta seção é mostrar como o processo de classificar fibrados pode ser encarado dentro da teoria de homotopia. A impossibilidade de se enunciar um resultado completamente geral nos leva a trabalhar com a classe dos fibrados enumeráveis, que é geral o bastante para o que nos propomos nesta tese. 
Uma cobertura aberta $\left\{U_{i}\right\}_{i \in J}$ de um espaço topológico $B$ é enumerável se existe uma partição da unidade localmente finita $\left\{u_{i}\right\}_{i \in J}$ tal que $u_{i}^{-1}((0,1]) \subseteq U_{i}$ para cada $i \in J$

Sabemos que um espaço de Hausdorff $B$ é paracompacto se e somente se toda cobertura aberta é enumerável. Um $G$-fibrado principal $\xi$ sobre um espaço $B$ é enumerável se é localmente trivial sobre uma cobertura aberta enumerável de $B$ observe que todo $G$-fibrado principal sobre um espaço paracompacto é enumerável.

Seja HTop a categoria cujos objetos são espaços topológicos e os morfismos são classes de equivalência de homotopias. Para cada espaço $B$ existe um funtor contravariante na categoria dos conjuntos

$$
[-, B]: \text { HTop } \rightarrow \text { Set }
$$

que leva um espaço $X$ no conjunto das classes de equivalência de aplicações homotópicas $[X, B]$ e um morfismo $f \in H T o p(X, Y)$ em uma aplicação

$$
\begin{aligned}
f^{*}:[Y, B] & \rightarrow[X, B], \\
f^{*}([h]) & =[h f]
\end{aligned}
$$

Proposição 4. Seja $p: E \rightarrow B$ um $G$-fibrado principal enumerável e $f, g: A \rightarrow B$ aplicações dadas. Então $f$ homotópica a $g$ implica $f^{-1}(p)=g^{-1}(p)$.

Esta proposição nos permite introduzir o funtor contravariante $N P_{G}: H T o p \rightarrow$ Set que leva um espaço $B$ no conjunto $N P_{G}(B)$ de todas as classes de equivalência de $G$-fibrados principais enumeráveis sobre $B$ e o morfismo $[f] \in \operatorname{HTop}(A, B)$ na função

$$
\begin{gathered}
N P_{G}([f]) N P_{G}([f]): N P_{G}(B) \rightarrow N P_{G}(A) \\
N P_{G}([f]): \xi \rightarrow f^{-1}(\xi) .
\end{gathered}
$$

Então, seja $\tilde{\xi}=(\tilde{E}, \tilde{p}, \tilde{B}, G)$ um $G$-fibrado principal enumerável fixado. Então existe uma transformação natural $\tau:[-, \tilde{B}] \rightarrow N P_{G}$ tal que para todo $B \in H T o p$,

$$
\begin{gathered}
\tau(B):[B, \tilde{B}] \mapsto N P_{G}(B) \\
{[f] \mapsto f^{-1}(\tilde{\xi}) .}
\end{gathered}
$$

Assim, finalmente estamos em condições de enunciar o seguinte teorema de classificação: 
Teorema 1.1. Se Ẽ é contrátil, então os funtores $N P_{G}$ e $[-, \tilde{B}]$ de HTop até a categoria dos conjuntos Set são naturalmente equivalentes.

A prova deste teorema pode ser encontrada em [9] 4.10, 4.12. Um $G$-fibrado principal enumerável com espaço total contrátil é chamado $G$-fibrado universal; O espaço base de tal fibrado é chamado de espaço classificante de $G$.

É claro a partir do teorema acima que quando é dado um $G$-fibrado universal, então para cada espaço $B$ existe uma bijeção entre os conjuntos $[B, \tilde{B}]$ e $N P_{G}(B)$. Esta equivalência é o que chamamos de classificação por homotopia de $G$-fibrados principais enumeráveis.

A necessidade da contractibilidade pode ser enfraquecida para obtermos uma classificação de fibrados até uma determinada dimensão fixa, como na seguinte proposição:

Proposição 5. se $\tilde{E}$ é n-conexo (isto é, se os primeiros n grupos de homotopia são triviais), então os funtores $N P_{G}$ e $[-, \tilde{B}]$ são naturalmente equivalentes para a categoria dos $C W$-complexos de dimensão no máximo $n$.

Um $G$-fibrado principal com espaço total $n$-conexo é chamado de fibrado $n$ universal. Isso significa que podemos classificar todos os $G$-fibrados principais sobre CW-complexos (em particular, variedades) de dimensão menor que $n$ usando os fibrados definidos em 1.2.

\subsubsection{Classes características e soma de Whitney}

As classes características são fundamentais para nossa classificação por serem um meio pelo qual diferenciamos os fibrados uns dos outros. Em 1935, E. Stiefel e H. Whitney introduziram este conceito com o intuito de resolver o problema de determinar o número de campos vetoriais linearmente independentes em uma variedade em um dado fibrado vetorial. Esta seção também introduz a soma de Whitney. Maiores detalhes sobre o que veremos aqui podem ser encontradas em [9].

Definição 1.12. Dados dois fibrados $\xi_{1}=\left(E_{1}, p_{1}, B\right)$ e $\xi_{2}=\left(E_{2}, p_{2}, B\right)$ sobre $B$, definimos o fibrado produto de $\xi_{1}$ e $\xi_{2}$ como sendo o fibrado $\xi_{1} \oplus \xi_{2}=\left(E_{1} \oplus E_{2}, q, B\right)$ com fibra $p_{1}^{-1}\left(e_{1}\right) \times p_{2}^{-1}\left(e_{2}\right)$, onde $E_{1} \oplus E_{2}=\left\{\left(e_{1}, e_{2}\right) \in E_{1} \times E_{2}\right.$ tal que $p_{1}\left(e_{1}\right)=$ $\left.p_{2}\left(e_{2}\right)\right\}$ e $q\left(e_{1}, e_{2}\right)=p_{1}\left(e_{1}\right)=p_{2}\left(e_{2}\right)$.

Observação 1.1. Se $\left(E_{1}, p_{1}, B\right)$ e $\left(E_{2}, p_{2}, B\right)$ são fibrados triviais com fibras $F_{1}$ e $F_{2}$ respectivamente, então $\left(E_{1}, p_{1}, B\right) \oplus\left(E_{2}, p_{2}, B\right)$ é um fibrado trivial com fibra $F_{1} \times F_{2}$. 
A definição e a observação acima quando aplicadas ao caso dos fibrados vetoriais também são válidas. Por questão de praticidade, quando o fibrado produto for utilizado em fibrados vetoriais, este será chamado de soma de Whitney. Neste caso, a fibra obtida é um espaço vetorial que tem a estrutura da soma direta das duas fibras-produto.

Valem as seguintes propriedades, onde $\zeta$ é um fibrado vetorial sobre $B$ :

$$
\xi \oplus \eta \approx \eta \oplus \xi \quad \text { e } \quad \xi \oplus(\eta \oplus \zeta) \approx(\xi \oplus \eta) \oplus \zeta .
$$

Como pode ser visto em [9], a toda aplicação contínua entre espaços vetoriais corresponde uma operação em fibrados vetoriais. Portanto, como o funtor produto tensorial é contínuo, temos então um produto tensorial $\xi \otimes \eta$ de dois fibrados vetoriais sobre $B$. Este produto tem as seguintes propriedades:

$$
\begin{array}{lc}
\xi \otimes \eta \approx \eta \otimes \xi \quad \text { e } \quad \xi \otimes(\eta \otimes \zeta) \approx(\xi \otimes \eta) \otimes \zeta \\
\text { e também } \quad \xi \otimes(\eta \oplus \zeta) \approx(\xi \otimes \eta) \oplus(\xi \otimes \zeta)
\end{array}
$$

e ainda se $\theta^{n}$ é o n-fibrado trivial sobre $B$, temos também:

$$
\xi \otimes \theta^{1} \approx \xi
$$

Dois fibrados vetoriais $\xi$ e $\eta$ são chamados $s$-equivalentes se $\xi \oplus \theta^{n}$ e $\eta \oplus \theta^{m}$ são isomorfos para algum $n$ e algum $m$. Chamamos esta propriedade de equivalência estável ou s-equivalência.

Definição 1.13. Uma aplicação de classificação de um fibrado vetorial $\xi$ sobre um espaço $X$ é um aplicação $f: X \rightarrow G_{K}\left(F^{k+m}\right)$ tal que $\xi$ e $f^{*}\left(\gamma_{K}^{k+m}\right)$ são isomorfos, onde $\gamma_{k}^{n}=\left(E_{1}, p_{1}, G_{K}\left(F^{n}\right)\right)$, sendo $G_{K}\left(F^{n}\right)$ uma grassmaniana como definida logo no início desta tese. ( $f$ é a aplicação cujo fibrado $\xi$ é um pull-back.)

Teorema 1.2. Se $f: X \rightarrow G_{K}\left(F^{n}\right)$ é uma aplicação de classificação para $\xi$ e se $g: X \rightarrow G_{l}\left(F^{m}\right)$ é uma aplicação de classificação para $\eta$, então a diagonal $d(f \times g) \Delta$ é uma aplicação de classificação para $\xi \oplus \eta$.

Daqui por diante, $c$ denota 1 no caso real e 2 no caso complexo, enquanto $K_{1}$ denota $\mathbb{Z}_{2}, K_{2}$ denota $\mathbb{Z}$ e $F$ denota $\mathbb{R}$ ou $\mathbb{C}$ para fibrados vetoriais reais ou complexos, respectivamente.

Observação 1.2. $O$ anel de cohomologia dos espaços projetivos $H^{*}\left(F P^{\infty}, K_{c}\right)$ é um anel de polinômios $K_{c}[z]$ onde grau $(z)=c$. 
Dado um fibrado vetorial $n$-dimensional $\xi=(E, p, B)$, o fibrado $P \xi=\left(E^{\prime}, q, B\right)$ obtido a partir de $\xi$ e cuja fibra é $F P^{n-1}$ é chamado de fibrado projetivo associado a $\xi$. Um ponto em $E^{\prime}$ é uma reta $L$ na fibra de $\xi$ sobre $q(L)$. O fibrado associado $q^{*}(\xi)$ tem um fibrado por linhas canônico $\lambda_{\xi}$ como subfibrado, onde um ponto no espaço total $E\left(\lambda_{\xi}\right)$ de $\lambda_{\xi}$ sobre $L$ é um par $(L, x)$ onde $q(L)=b=p(x)$, ou seja, $x \in L$.

Vamos admitir daqui por diante que $E(P \xi)=E^{\prime}$ é paracompacto. Então existe pelo teorema de classificação 1.1 uma aplicação $f: E(P \xi) \rightarrow F P^{\infty}$ tal que $f^{*}\left(\gamma_{1}\right) \approx$ $\lambda_{\xi}^{*}$, onde $\lambda_{\xi}^{*}$ é o fibrado conjugado a $\lambda_{\xi}$ e $\gamma_{1}$ é o fibrado por linhas universal.

Seja $a_{\xi}$ a classe $f^{*}(z)$. Como $f$ é única a menos de homotopia, $a_{\xi}$ é bem definida. O teorema a seguir caracteriza $a_{\xi}$ :

Teorema 1.3. Para um fibrado vetorial $n$-dimensional $\xi$, as classes $1, a_{\xi}, \ldots, a_{\xi}^{n-1}$ formam uma base do $H^{*}\left(V(\xi), K_{c}\right)$-módulo $H^{*}\left(E(P(\xi)), K_{c}\right)$. Mais ainda, $p^{*}$ : $H^{*}(B(\xi)) \rightarrow H^{*}(E(P(\xi)))$ é injetora.

Este teorema nos garante que existe um único $x_{i}(\xi) \in H^{c i}\left(B, K_{c}\right)$ tal que $a_{\xi}^{n}=-\sum_{1 \leq i \leq n} x_{i}(\xi) a_{\xi}^{n-i}$, e portanto podemos escrever $x(\xi)=1+x_{1}(\xi)+\ldots+x_{n}(\xi)$.

Definição 1.14. Para um fibrado vetorial real $\xi$, a i-ésima classe de Stiefel-Whitney de $\xi$, denotada por $w_{i}(\xi)$ é $x_{i}(\xi) \in H^{i}(B(\xi), \mathbb{Z})$. Mais ainda, $w(\xi)=1+w_{1}(\xi)+\ldots+$ $w_{n}(\xi)$ e $c(\xi)=1+c_{1}(\xi)+\ldots+c_{n}(\xi)$ são chamadas de classe total de Stiefel-Whitney e classe total de Chern, respectivamente.

As propriedades que veremos a seguir caracterizam completamente as classes de Stiefel-Whitney e de Chern - maiores detalhes em [14].

Para cada fibrado vetorial real $\xi$ sobre um espaço $B$ existe uma classe $w(\xi) \in$ $H^{*}\left(B, \mathbb{Z}_{2}\right)$ chamada de classe de Stiefel-Whitney com as seguintes propriedades:

1. Temos $w(\xi)=1+w_{1}(\xi)+\ldots+w_{n}(\xi)$, onde $w_{i}(x) \in H^{i}\left(B, \mathbb{Z}_{2}\right)$ e $w_{i}(\xi)=0$ para $i>\operatorname{dim} \xi$.

2. Se $\xi$ e $\eta$ são $B$-isomorfos, segue que $w(\xi)=w(\eta)$ e se $f: B_{1} \rightarrow B$ é uma aplicação, então temos $f^{*}(w(\xi))=w\left(f^{*}(\xi)\right)$. 
3. Para dois fibrados vetoriais $\xi$ e $\eta$ sobre $B$, a relação $w(\xi \oplus \eta)=w(\xi) w(\eta)$ vale, onde o produto é o produto cup.

4. Para o fibrado por linhas canônico $\lambda$ sobre $S^{1}=\mathbb{R} P^{1}$, o elemento $w_{1}(\lambda)$ é não-nulo em $H^{1}\left(S^{1}, \mathbb{Z}_{2}\right)=\mathbb{Z}_{2}$.

5. Para o fibrado por linhas canônico $\lambda_{1}$ sobre $\mathbb{R} P^{\infty}$, o elemento $w_{1}\left(\gamma_{1}\right)$ é o gerador do anel polinomial $H^{*}\left(\mathbb{R} P^{\infty}, \mathbb{Z}_{2}\right)$. (Esta propriedade é equivalente à anterior.)

Podemos então escolher um gerador $z$ de $H^{2}\left(S^{2}, \mathbb{Z}\right)$ que determina também um elemento gerador de $H^{2}(\mathbb{C} P, \mathbb{Z})$ para cada $n$ com $1 \leq n \leq \infty$ de tal forma que $z$ gera o anel polinomial $H^{*}\left(\mathbb{C} P^{\infty}, \mathbb{Z}\right)$.

Por outro lado, para cada fibrado vetorial complexo $\xi$ sobre um espaço $B$ existe uma classe $c(\xi) \in H^{*}(B, \mathbb{Z})$ chamada de classe de Chern com as seguintes propriedades:

1. Temos $c(\xi)=1+c_{1}(\xi)+\ldots+c_{n}(\xi)$, onde $c_{i}(x) \in H^{i}(B, \mathbb{Z})$ e $c_{i}(\xi)=0$ para $i>\operatorname{dim} \xi$.

2. Se $\xi$ e $\eta$ são $B$-isomorfos, segue que $c(\xi)=c(\eta)$ e se $f: B_{1} \rightarrow B$ é uma aplicação, então temos $f^{*}(c(\xi))=c\left(f^{*}(\xi)\right)$.

3. Para dois fibrados vetoriais $\xi$ e $\eta$ sobre $B$, a relação $c(\xi \oplus \eta)=c(\xi) c(\eta)$ vale, onde o produto é o produto cup.

4. Para o fibrado por linhas canônico $\lambda$ sobre $S^{2}=\mathbb{C} P^{1}$, o elemento $c_{1}(\lambda)$ é o gerador de $H^{2}\left(S^{2}, \mathbb{Z}\right)=\mathbb{Z}$.

5. Para o fibrado por linhas canônico $\lambda_{1}$ sobre $\mathbb{C} P^{\infty}$, o elemento $c_{1}\left(\gamma_{1}\right)$ é o gerador $z$ do anel polinomial $H^{*}\left(\mathbb{C} P^{\infty}, \mathbb{Z}\right)$. (Esta propriedade é equivalente à anterior.)

Teorema 1.4. Se $\xi$ é um fibrado trivial sobre $B$, então $w_{i}(\xi)=0$ para $i>0$ no caso real e $c_{i}(\xi)=0$ para $i>0$ no caso complexo.

Teorema 1.5. Sejam $\xi$ e $\eta$ dois fibrados vetoriais s-equivalentes. Então a relação $w(\xi)=w(\eta)$ vale no caso real e $c(\xi)=c(\eta)$ vale no caso complexo. 
Note que com isso, as classes características não distinguem fibrados $s$-equivalentes. Uma outra maneira de se ver as classes características (segundo Steenrod em [21]) é a seguinte: seja $G_{k}\left(\mathbb{R}^{n}\right)$ a grassmaniana dos subespaços $k$-dimensionais em $\mathbb{R}^{n}$ e seja $M$ uma variedade $m$-dimensional. Suponha que $M$ possa ser triangularizada e que exista um fibrado vetorial real $n$-dimensional sobre $M$. É possível então associarmos $k$ vetores linearmente independentes a cada vértice da triangularização. Assim, temos uma aplicação $M^{0} \rightarrow G_{k}\left(\mathbb{R}^{n}\right)$ que leva cada vértice do 0-esqueleto de $M$ ao subespaço $k$-dimensional gerado pelos $k$ vetores associados a este vértice. $\mathrm{O}$ problema então é estender esta associação de $k$ vetores linearmente independentes ao 1-esqueleto de $M$, e assim por diante, uma dimensão por vez. A obstrução a estender uma associação do $(i-1)$-esqueleto para o $i$-esqueleto é na verdade um cociclo em $C^{i}\left(M, \pi_{i-1}\left(G_{k}\left(\mathbb{R}^{n}\right)\right)\right)$ - as cocadeias em $M$ com coeficientes no sistema de coeficientes locais $\pi_{i-1}\left(G_{k}\right.$ (espaço vetorial em cada ponto de $\left.\left.M\right)\right)$. Reduzindo para mod 2 se necessário, estas classes de obstrução determinam classes $w_{i} \in H^{i}(M, \mathbb{Z} / 2)$, que são exatamente as classes de Stiefel-Whitney para o fibrado considerado. 


\section{Capítulo 2}

\section{Coeficientes locais e resoluções}

Neste capítulo trataremos de resoluções e de sheaves, em particular de um tipo especial de sheaf chamado de sistema de coeficientes locais (ou ainda fibrado de grupos). Daremos atenção especial ao sistema de coeficientes locais utilizado no capítulo final, inclusive calculando a cohomologia dos espaços projetivos com coeficientes neste sistema.

\subsection{Sheaves}

Um sheaf é, essencialmente, um sistema de coeficientes sobre um dado espaço $X$ que associa um grupo (ou módulo) para cada $x \in X$. Maiores detalhes podem ser encontrados em [24], mas de maneira formal, podemos definir:

Definição 2.1. Um sheaf é uma terna $(S, X, p)$ onde $S$ é o espaço total (que também é chamado de sheaf), $X$ é o espaço base e p é uma projeção contínua e sobrejetora de $S$ em $X$. Mais ainda, a terna $(S, X, p)$ precisa que:

1. $p$ seja um homeomorfismo local, ou seja, para cada $x \in S$ existe um aberto $U \subset S$ que contem $x$ e tal que $\left.p\right|_{U}: U \rightarrow V$ seja um homeomorfismo de $U$ com um aberto $V$ de $X$ que contém $p(x)$.

2. Exista um anel comutativo $K$ com unidade tal que todo conjunto discreto $S_{x}=$ $p^{-1}(x)$ (chamado Stalk sobre $x$ ) tenha uma estrutura de K-módulo.

3. Sejam contínuas as seguintes aplicações:

$\bar{\lambda}: S \rightarrow S$ induzida para todo $\lambda \in K$ por $\lambda: S_{x} \rightarrow S_{x}$ tal que $\lambda(x)=\lambda x$.

$\mp: S \times S \rightarrow S$ induzida pela soma nos $K$-módulos $S_{x}$. 
Observe ainda que dados dois sheaves $(S, X, p)$ e $\left(T, X, p^{\prime}\right)$, temos então um sheaf $\left(S+T, X, p^{\prime \prime}\right)$ onde $S+T=\left\{(x, y) \in S \times T\right.$ tal que $\left.p(x)=p^{\prime}(y)\right\}$ e $p^{\prime \prime}: S+T \rightarrow X$ é dada por $p^{\prime \prime}(x, y)=p(x)=p^{\prime}(y)$.

\section{Exemplo 2.1.}

- Seja $M$ um K-módulo com a topologia discreta. Seja $S=X \times M$ e $p: S \rightarrow X$ a projeção $p(x, m)=x$. Este sheaf é chamado de sheaf constante, e o stalk sobre $y \in X$ é $\{y\} \times M$.

- Considere um fibrado sobre um espaço $X$ onde a fibra é um grupo abeliano e o grupo estrutural do fibrado é totalmente desconexo. Este sheaf de $\mathbb{Z}$-módulos é também conhecido como fibrado de grupos.

\subsubsection{Sistemas de coeficientes locais.}

Esta seção introduz um tipo de sheaf chamado de sistema de coeficientes locais. Faremos como em [6] e mostraremos como podemos definir a homologia com coeficientes torcidos a partir de um sistema como este:

Definição 2.2. Um sistema de coeficientes locais sobre $X$ é um fibrado sobre $X$ cujas fibras são grupos abelianos discretos e cujas funções de transição são automorfismos destes grupos.

Muito importante para os próximos capítulos é o seguinte caso particular:

Definição 2.3. Um grupo torcido é um par ordenado $(G, T)$ onde $G$ é um grupo abeliano e $T: G \rightarrow G$ é um homomorfismo de ordem $2 \mathrm{em} G$. Se $X$ é um espaço, um $(\mathbf{G}, \mathbf{T})$-sheaf sobre $\mathbf{X}$ é um fibrado sobre $X$ com fibra $G$ e grupo estrutural $\mathbb{Z}_{2}$, sendo que a identidade $\mathbb{1}$ corresponde a $T^{2}$ e o elemento não trivial corresponde a $T$.

Exemplo 2.2. Seja $\mathbf{G}^{\mathbf{T}}[\mathbf{u}]$ o $(G, T)$-sheaf sobre $\mathbb{R} P^{\infty}=\cup_{n} \mathbb{R} P^{n}$ obtido pela identificação de $(x, g)$ com $\left(T^{\prime} x, T g\right)$ para todo $(x, g) \in S^{\infty} \times G$, onde $T^{\prime}: S^{\infty} \rightarrow S^{\infty}$ é a antipodal. Este exemplo é muito importante neste trabalho, como veremos nos próximos capítulos.

Note que $\mathbb{R} P^{\infty}$ também pode ser visto como o espaço das retas que passam pela origem em $\mathbb{R}^{\infty}=\cup_{n} \mathbb{R}^{n}$. Perceba ainda que ao identificarmos os pontos da $S^{\infty}$ obtemos $\mathbb{R} P^{\infty}$ e ao identificarmos os pontos de $G$ pela ação $T$ estamos colando as fibras daquilo que será o fibrado. Assim, depois de feita toda a colagem, temos um fibrado sobre $\mathbb{R} P^{\infty}$. 
Definição 2.4. Seja $a \in H^{1}\left(X, x_{0} ; \mathbb{Z}_{2}\right)$ e $f:\left(X, x_{0}\right) \rightarrow\left(\mathbb{R} P^{\infty}, *\right)$ uma aplicação tal que $f^{*}(u)=a$, onde " $u^{\prime \prime}$ é o gerador da cohomologia de $\mathbb{R} P^{\infty}$ no nivel 1 , também conhecido como classe fundamental de $\mathbb{R} P^{\infty}$. Definimos o sheaf $G^{T}[a]$ sobre $X$ como sendo o pull-back $f^{-1}\left(G^{T}[u]\right)$. Nestas condições, dizemos que " $a^{\prime \prime}$ é a classe de torção de $G^{T}[a]$.

Proposição 6. $G^{T}[a]$ é universal no sentido de Steenrod, isto é, se $H$ é um $(G, T)$ sheaf sobre um espaço $X$, então $H \approx G^{T}[a]$ para algum único a $\in H^{1}\left(X, x_{0} ; \mathbb{Z}_{2}\right)$.

As provas das proposições encontradas daqui até o fim desta seção fogem do escopo deste trabalho mas podem ser vistas com detalhes em [11].

Proposição 7. Se $S$ é um sheaf sobre um espaço $X$ e $A \subset X$ é fechado, $p: X \times I \rightarrow$ $X$ é a suspensão e $p^{-1} S$ é a notação para o pullback de $S$ por $p$, então existe um isomorfismo $S: H^{*}(X, A ; S) \rightarrow H^{*}\left(X \times I,(X \times \partial I) \cup(A \times I) ; p^{-1}(S)\right)$.

Definição 2.5. Se $S$ e $S^{\prime}$ são sheaves sobre $X$, dizemos que a seqüência exata curta $E: 0 \rightarrow S \stackrel{i}{\rightarrow} S^{\prime \prime} \stackrel{p}{\rightarrow} S^{\prime} \rightarrow 0$ é uma extensão de $S^{\prime}$ por $S$.

Se $0 \rightarrow A \rightarrow B \rightarrow C \rightarrow 0$ é uma seqüência exata curta de grupos abelianos, então $0 \rightarrow \operatorname{Hom}\left(C_{*} X, A\right) \rightarrow \operatorname{Hom}\left(C_{*} X, B\right) \rightarrow \operatorname{Hom}\left(C_{*} X, C\right) \rightarrow 0$ é uma seqüência exata - onde $C_{*} X$ denota o complexo de cadeias singulares de $X$. Desta maneira, obtemos uma seqüência longa exata de cohomologia

$$
\ldots \rightarrow H^{k}(X ; A) \rightarrow H^{k}(X ; B) \rightarrow H^{k}(X ; C) \rightarrow H^{k+1}(X ; A) \rightarrow \ldots
$$

Definição 2.6. Os homomorfismos de conexão $\beta_{k}: H^{k}(X ; C) \rightarrow H^{K+1}(X ; A)$ são chamados de homomorfismos de Bockstein ou simplesmente bockstein's associados à seqüência exata curta $0 \rightarrow A \rightarrow B \rightarrow C \rightarrow 0$.

Para mais informações sobre bockstein's, veja [4].

Seja $X$ um espaço, $A \subset X$ fechado. Se $\alpha: S \rightarrow S^{\prime}$ é um homomorfismo de sheaves sobre $X$, então temos um homomorfismo $\alpha^{*}: H^{*}(X, A ; S) \rightarrow H^{*}\left(X, A ; S^{\prime}\right)$. A partir daí temos que a seqüência exata curta $E$ determina uma seqüência exata longa

$$
\ldots \rightarrow H^{n}(X, A ; S) \stackrel{i_{*}}{\rightarrow} H^{n}\left(X, A ; S^{\prime \prime}\right) \stackrel{p_{*}}{\rightarrow} H^{n}\left(X, A ; S^{\prime}\right) \stackrel{\delta^{E}}{\rightarrow} H^{n+1}(X, A ; S) \rightarrow \ldots
$$

onde $\delta^{E}$ é o bockstein de $E$.

Proposição 8. Se $S$ e $S^{\prime}$ são sheaves sobre $X$ e ainda se $E: 0 \rightarrow S \stackrel{i}{\rightarrow} S^{\prime \prime} \stackrel{p}{\rightarrow} S^{\prime} \rightarrow 0$ e se $F: 0 \rightarrow S \stackrel{j}{\rightarrow} U \stackrel{q}{\rightarrow} S^{\prime} \rightarrow 0$ são elementos de $\operatorname{Ext}\left(S, S^{\prime}\right)$, então $\delta^{E+F}=\delta^{E}+\delta^{F}$. 
Antes de passarmos à frente, um breve comentário: como grupos abelianos, $\operatorname{Ext}\left(\mathbb{Z}_{2}, \mathbb{Z}_{2}\right) \approx \mathbb{Z}_{2}$, sendo $E^{0}: 0 \rightarrow \mathbb{Z}_{2} \rightarrow \mathbb{Z}_{2} \oplus \mathbb{Z}_{2} \rightarrow \mathbb{Z}_{2} \rightarrow 0$ e $E^{1}: 0 \rightarrow \mathbb{Z}_{2} \rightarrow$ $\mathbb{Z}_{4} \rightarrow \mathbb{Z}_{2} \rightarrow 0$.

Agora fixemos um espaço $X$. Estudaremos as extensões $(E x t)$ de sheaves sobre $X$ :

Proposição 9. Como sheaves sobre $X, \operatorname{Ext}\left(\mathbb{Z}_{2}, \mathbb{Z}_{2}\right) \approx \mathbb{Z}_{2} \oplus H^{1}\left(X, x_{0} ; \mathbb{Z}_{2}\right)$ onde, para qualquer $a \in H^{1}\left(X, x_{0}, \mathbb{Z}_{2}\right)$,

- $(0, a)$ corresponde a $E_{a}^{0}: 0 \rightarrow \mathbb{Z}_{2} \stackrel{i_{1}}{\rightarrow}\left(\mathbb{Z}_{2} \oplus \mathbb{Z}_{2}\right)^{T}[a] \stackrel{p_{2}}{\rightarrow} \mathbb{Z}_{2} \rightarrow 0$, sendo que $T(x, y)=(x+y, y), \quad i(x)=(x, 0), \quad$ e $p(x, y)=y$.

- $(1, a)$ corresponde a $E_{a}^{1}: 0 \rightarrow \mathbb{Z}_{2} \stackrel{m}{\rightarrow} \mathbb{Z}_{4}^{T}[a] \stackrel{e}{\rightarrow} \mathbb{Z}_{2} \rightarrow 0$, sendo que $T(x)=-x$ para todo $x \in \mathbb{Z}_{4}, m(1)=2$ e e $(1)=1$.

Temos então um diagrama comutativo com linhas exatas para todo $a \in H^{1}\left(X, x_{0}, \mathbb{Z}_{2}\right)$ :

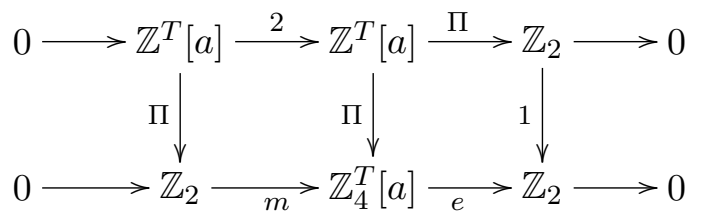

Seja $\beta^{T}[a]$ (ou $\beta^{T}$ quando não houver dúvida quanto à "a") a notação para o bockstein da linha de cima enquanto $\left(S_{q}^{1}\right)^{T} x$ (ou $\left.\left(S_{q}^{1}\right)^{T}\right)$ denota o bockstein da linha de baixo. Temos que $\Pi_{*} \beta^{T}=\left(S_{q}^{1}\right)^{T}$. (Observe que esta aplicação $\Pi$ é dada pela projeção da fibra $\mathbb{Z}$ na fibra $\mathbb{Z} / 2$ ou $\mathbb{Z} / 4$, sempre da maneira canônica.)

Proposição 10. Para todo $n \geq 0$ e qualquer $x \in H^{n}\left(X, A ; \mathbb{Z}_{2}\right),\left(S_{q}^{1}\right)^{T} x=S_{q}^{1} x+x \smile$ $a$.

Proposição 11. Para todo $n \geq 0$ e para todo $x \in H^{n}\left(X, A ; \mathbb{Z}_{2}\right), \delta(x)=x \smile$ a, onde $\delta$ é o bockstein de $E_{a}^{0}: 0 \rightarrow \mathbb{Z}_{2} \rightarrow\left(\mathbb{Z}_{2} \oplus \mathbb{Z}_{2}\right)^{T}[a] \rightarrow \mathbb{Z}_{2} \rightarrow 0$.

Seja (X,A) um par CW, e seja $a \in H^{1}\left(X, x_{0} ; \mathbb{Z}_{2}\right)$, com $\alpha=\left(\beta^{T}[a]\right)(\mathbb{1}) \in$ $H^{1}\left(X, A ; Z^{T}[a]\right)$ (onde $\beta$ é o mesmo bockstein há pouco definido e $\mathbb{1}$ é o gerador de $\left.H^{0}\left(X, Z ; \mathbb{Z}_{2}\right)\right)$. Então temos o seguinte diagrama comutativo, onde $i_{1}(x)=$ $(x, 0), T(x, y)=(y-x, y), j_{1}(x)=(x, 2 x)$ e $q_{2}(x, y)=y-2 x:$

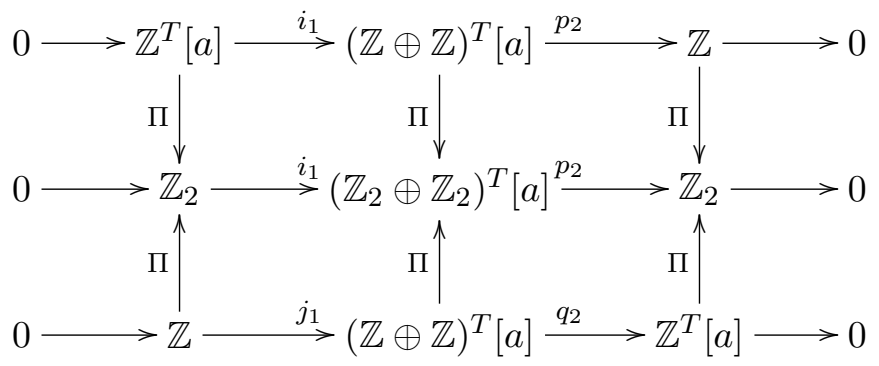


Proposição 12. Aplicar qualquer um dos homomorfismos Bockstein $\delta_{1}$ e $\delta_{2}$ relativos à primeira e à última linha do diagrama anterior é equivalente a se tomar o produto cup $\operatorname{com} \alpha$.

Nosso objetivo ao final desta seção é dar um exemplo do cálculo da cohomologia de um determinado espaço (no caso, $\mathbb{R} P^{n}$ ) com coeficientes em um dado sheaf. Para isso, faremos agora uma breve exposição sobre a homologia celular do espaço $\mathbb{R} P^{n}$ e logo a seguir trataremos de reunir todos os resultados da seção de maneira a apresentá-los de forma mais prática.

Sabemos que $\mathbb{R} P_{k} \sim S^{k} / A \sim D^{k} / A_{\partial D^{k}}$, sendo que $\partial D^{k}$ com seus pontos identificados pela aplicação antipodal $A$ é $\mathbb{R} P_{k-1}$. Então $\mathbb{R} P_{k}=\mathbb{R} P_{k-1} \cup_{\varphi} D^{n}$, onde $\varphi: S^{n-1} \rightarrow \mathbb{R} P_{k-1}$ leva cada ponto de $S^{n-1}$ até sua projeção em $\mathbb{R} P_{k-1}$. Segue por indução sobre $n$ que $\mathbb{R} P_{k}$ tem uma estrutura de CW-complexo $e^{0} \cup e^{1} \cup e^{2} \cup \ldots \cup e^{k}$ com uma célula $e^{i}$ em cada dimensão $i \leq k$.

A seguinte proposição é um lema cuja prova pode ser encontrada em [6]:

Proposição 13. Se $X$ é um $C W$-complexo e $X^{n}$ é seu n-esqueleto, então:

1. $H_{k}\left(X^{n}, X^{n-1}\right)=0$ para $k \neq n$ e é livre abeliano para $k=n$, com base em bijeção com as n-células de $X$.

2. $H_{k}\left(X^{n}\right)=0$ para $k>n$. Em particular, se $X$ é de dimensão finita, então $H_{k}(X)=0$ para $k>\operatorname{dim} X$.

3. A inclusão $i: X^{n} \rightarrow X$ induz isomorfismo $i_{*}: H_{k}\left(X^{n}\right) \rightarrow H_{k}(X)$ se $k<n$.

O primeiro item desta proposição é importante para entendermos o significado geométrico da homologia celular. A sua prova é simples:

Demonstração. Como $X^{n}$ é um CW-complexo e $X^{n-1}$ é um subcomplexo não vazio, então o par $\left(X^{n}, X^{n-1}\right)$ é um "bom par" e como tal, tem a propriedade de que $H_{n}\left(X^{n}, X^{n-1}\right) \approx H_{n}\left(X^{n} / X^{n-1}, X^{n-1} / X^{n-1}\right) \approx \widetilde{H_{n}}\left(X^{n} / X^{n-1}\right)$.

Corolário 1. É claro que $X^{n} / X^{n-1}$ é o produto edge (união por um ponto) de $n$ esferas $S^{n}$, uma para cada n-célula de $X$, então temos um isomorfismo $\oplus_{\alpha} \widetilde{H_{n}}\left(S_{\alpha}^{n}\right) \approx$ $\widetilde{H_{n}}\left(X^{n} / X^{n-1}\right) \approx H_{n}\left(X^{n}, X^{n-1}\right)$.

Seja $X$ um CW-complexo. Usando a proposição anterior e partes das seqüências exatas longas dos pares $\left(X^{n+1}, X^{n}\right),\left(X^{n}, X^{n-1}\right),\left(X^{n-1}, X^{n-2}\right)$ temos um diagrama como o da figura abaixo, no qual a linha horizontal é o complexo celular de cadeias de $X$ e onde $d_{n+1}=j_{n} \circ \partial_{n+1}$ e $d_{n}=j_{n-1} \circ \partial_{n}$, com $d_{n} d_{n+1}=0$ : 


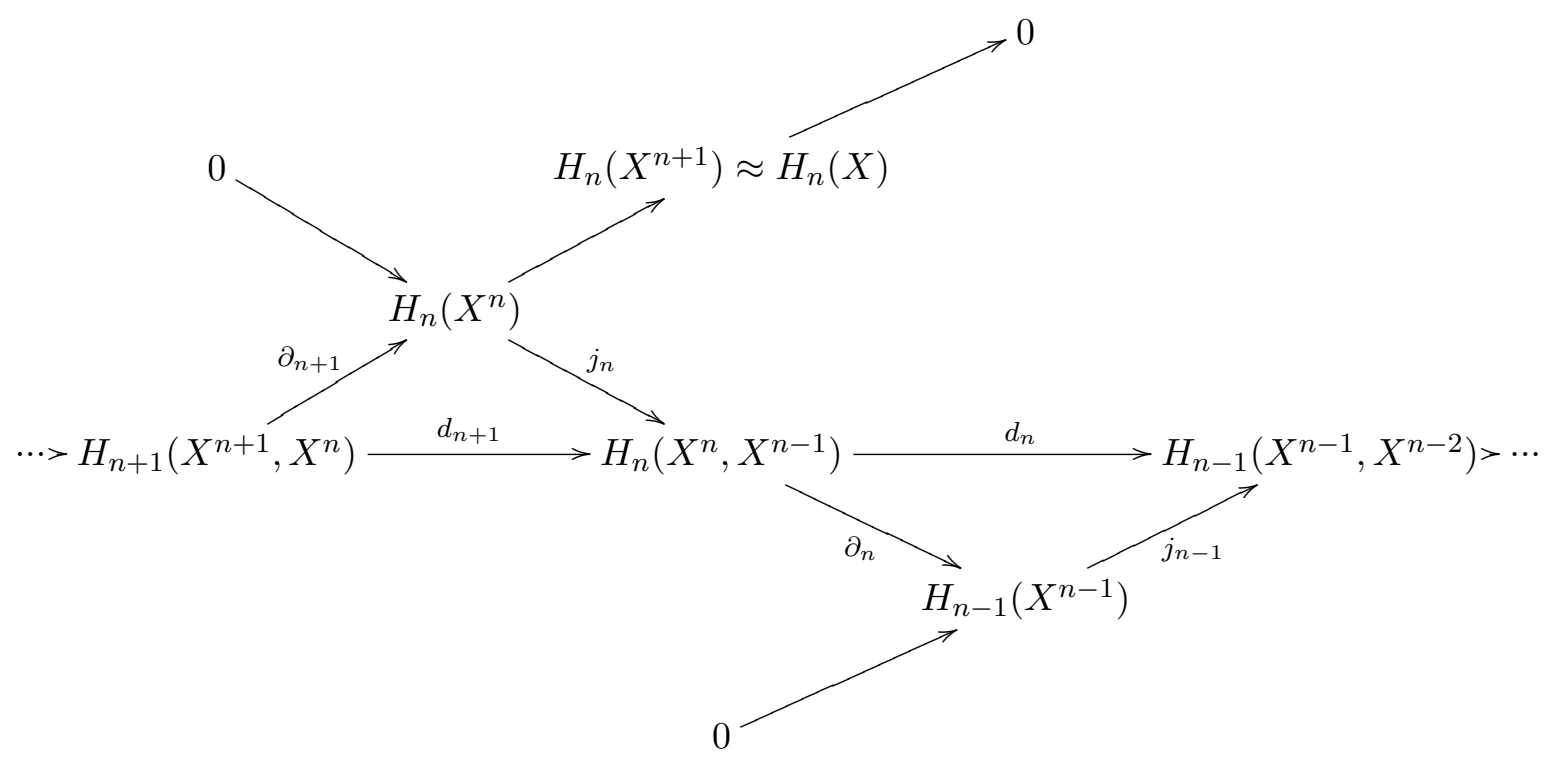

Observação 2.1. A aplicação bordo $d_{n}$ pode ser dada de maneira mais explícita: para $n=1$ temos que $d_{1}$ é igual à aplicação bordo simplicial $\partial_{1}$, e para $n>1$ podemos ver $d_{n}$ como sendo $d_{n}\left(e_{\alpha}^{n}\right)=\sum_{\beta} d_{\alpha \beta} e_{\beta}^{n-1}$, onde $d_{\alpha \beta}$ é o grau da aplicação $S_{\alpha}^{n-1} \rightarrow$ $X^{n-1} \rightarrow S_{\beta}^{n-1}$ que é a composição da aplicação de colagem $\varphi_{e_{\alpha}^{n}}$ com a aplicação quociente que colapsa $X^{n-1}-e_{\beta}^{n-1}$ a um ponto.

Ainda de acordo com a proposição anterior, podemos pensar nos elementos de $H_{n}\left(X^{n}, X^{n-1}\right)$ como combinações lineares das n-células de $X$. A homologia correspondente é a homologia celular de $X$.

Antes de apresentar o cálculo de cohomologias de $\mathbb{R} P_{k}$ com coeficientes em $\mathbb{Z}^{T}[u]$, vamos primeiro analisar o cálculo da homologia de $\mathbb{R} P_{k}$ com coeficientes em $\mathbb{Z}$ usando a homologia celular.

Proposição 14. A homologia de $\mathbb{R} P_{k}$ com coeficientes em $\mathbb{Z}$ é dada por:

$$
H_{n}\left(\mathbb{R} P_{k}, \mathbb{Z}\right)= \begin{cases}\mathbb{Z} & \text { Se } n=0 \text { ou } n=k \text { ímpar } ; \\ \mathbb{Z}_{2} & \text { Se n é impar com } 0<n<k ; \\ 0 & \text { Caso contrário. }\end{cases}
$$

Demonstração. Como vimos, $\mathbb{R} P_{k}$ tem uma estrutura de CW-complexo com uma célula $e^{i}$ em cada dimensão $i \leq k$, e a aplicação de colagem para $e^{k}$ é o recobrimento 
de duas folhas $\varphi: S^{k-1} \rightarrow \mathbb{R} P_{k}$. Para calcular a aplicação bordo $d_{k}$, calculamos o grau da composição $S^{k-1} \stackrel{\varphi}{\rightarrow} \mathbb{R} P_{k-1}=D^{k-1} \cup_{\partial} \mathbb{R} P_{k-2} \stackrel{q}{\rightarrow} \mathbb{R} P_{k-1} / \mathbb{R} P_{k-2} \approx S^{k-1}$, onde $q$ é a aplicação quociente.

A aplicação $q \varphi$ é um homeomorfismo quando restrita a cada componente de $S^{k-1}-S^{k-2}$, e estes dois homeomorfismos são obtidos um do outro por composição com a antipodal de $s^{k-1}$, que tem grau $(-1)^{k}$.

Segue que $\operatorname{grau}(q \varphi)=\operatorname{grau} \mathbb{1}+\operatorname{grau}(-\mathbb{1}=A)$, e portanto $d_{k}$ é ou 0 ou multiplicação por 2 , respectivamente se $k$ é ímpar ou par. Assim o complexo celular de cadeias para $\mathbb{R} P_{k}$ é:

$$
0 \longrightarrow \mathbb{Z}^{1+\stackrel{(-1)^{k}}{\longrightarrow}} \mathbb{Z} \longrightarrow \ldots \longrightarrow \mathbb{Z} \stackrel{2}{\longrightarrow} \mathbb{Z} \stackrel{0}{\longrightarrow} \mathbb{Z} \stackrel{2}{\longrightarrow} \mathbb{Z} \stackrel{0}{\longrightarrow} 0
$$

A partir daí é fácil calcular os grupos de homologia para $\mathbb{R} P_{k}$, como queríamos.

E sabemos também a cohomologia de $\mathbb{R} P^{k}$ com coeficientes em $\mathbb{Z}$ :

$$
H^{n}\left(\mathbb{R} P_{k} ; \mathbb{Z}\right) \approx \begin{cases}\mathbb{Z}_{2}, \text { gerado por } u^{n}, & \text { se } n \text { é par com } 0<n \leq k ; \\ \mathbb{Z}, \text { gerado por } \mathbb{1}, & \text { se } n=0 ; \\ 0, & \text { se } n \text { é ímpar, } 0<n<k ; \\ \mathbb{Z}, \text { gerado pela classe top } t\left(\mathbb{R} P_{k}\right) & \text { se } n=k \text { ímpar; } \\ 0 & \text { se } n>k .\end{cases}
$$

Mais ainda, fazendo uso do teorema dos coeficientes universais, temos a versão com coeficientes em $\mathbb{Z}_{2}$ :

$$
H^{n}\left(\mathbb{R} P_{k} ; \mathbb{Z}_{2}\right) \approx \begin{cases}\mathbb{Z}_{2}, \text { gerado por } u^{n} & \text { se } n \leq k \\ 0 & \text { se } n>k\end{cases}
$$

\subsection{Homologia com coeficientes locais.}

No capítulo final deste trabalho os tradicionais sistemas de coeficientes globais para homologia e cohomologia não são suficientes para descrever as situações por lá encontradas. Esta seção pretende apresentar uma solução para este problema. 
Seja $E$ um sistema de coeficientes locais sobre $X$. Queremos definir a homologia simplicial local de $X$ com coeficientes no fibrado $E$.

Seja $\sigma=x_{0} x_{1} \ldots x_{n}$ um $n$-simplexo simplicial ordenado, e denote por $v_{\sigma}$ o seu vértice líder $x_{0}$. Note que para qualquer face $\partial_{i} \sigma \operatorname{com} i \neq 0$ temos que $v_{\partial_{i} \sigma}=x_{0}=v_{\sigma}$, mas para $i=0$ temos que $v_{\partial_{0} \sigma}=x_{1}$ porque $\partial_{0} \sigma=x_{1} x_{2} \ldots x_{n}$.

Definição 2.7. Definimos então o grupo $\Sigma_{n}(X, E)$ das n-cadeias simpliciais como sendo o grupo aditivo de todas as somas formais finitas

$$
\sum_{i=1}^{m} \alpha_{i} x_{i}
$$

onde $x_{i}$ são simplexos simpliciais ordenados de $X$ e $\alpha_{i}$ pertence à fibra do vértice líder $v_{x_{i}}$.

A maneira natural de pensar o operador bordo seria considerá-lo como sendo, para um elemento básico $\alpha x$, algo como $\partial x=\sum_{i=0}^{n}(-i)^{i} \alpha \partial_{i} x$. Mas pelo que vimos acima, temos que $\alpha$ não pertence à fibra no ponto $x_{0}$ e portanto não teríamos uma soma formal como aquela definida no parágrafo anterior. Para contornamos este problema, faremos uso do isomorfismo $T_{x_{0} x_{1}}^{*}$ entre as fibras dos pontos extremos determinados pelo caminho $T_{x_{0} x_{1}}:=t x_{1}+(1-t) x_{0}$, como mostrado logo a seguir.

Definição 2.8. O operador bordo em um elemento básico $\alpha x$ é definido da seguinte maneira, onde $\partial: \Sigma_{n}(X, E) \rightarrow \Sigma_{n-1}(X, E)$ :

$$
\partial(\alpha x)=T_{x_{0} x_{1}}^{*}(\alpha) \partial_{0} x+\sum_{i=1}^{n}(-1)^{i} \alpha \partial_{i} x
$$

Desta forma, temos que $\Sigma_{*}(X, E)$ é um complexo de cadeias e sua homologia é a dos coeficientes locais em $X$ com os coeficientes em $E$. A seguinte proposição é um exemplo de como calcular tal homologia.

Proposição 15. A homologia simplicial de $S^{1}$ com coeficientes no fibrado $E=$ $\left(E, p, S^{1}, \mathbb{Z}, \mathbb{Z}_{2}\right)$ é dada por $H_{0}\left(S^{1}, E\right)=\mathbb{Z}_{2}$ e $H_{1}\left(S^{1}, E\right)=0$.

Demonstração. Tomemos a decomposição simplicial de $S^{1}$ que nos fornece a seguinte seqüência: $0 \rightarrow C_{1} \stackrel{\partial}{\rightarrow} C_{0} \rightarrow 0$, onde $C_{0}=<x_{0}, x_{1}, x_{2}>$ e $C_{1}=<x_{0} x_{1}, x_{1} x_{2}, x_{2}, x_{0}>$. Conseqüentemente $Z_{0}=C_{0}$ e também $B_{1}$ é trivial. Nosso próximo passo é analisar o operador bordo $\partial: C_{1} \rightarrow C_{0}$. Note que

$$
\partial\left(\sum_{i=0}^{2} \alpha_{i}\left(x_{i} x_{i+1}\right)\right)=\sum_{i=0}^{2} \partial\left(\alpha_{i}\left(x_{i} x_{i+1}\right)\right)
$$


Neste ponto é necessário fixar os homomorfismos $T_{x_{i} x_{i+1}}^{*}$ para $\mathrm{i}=0,1,2$. Fixaremos tais homomorfismos com base no conhecimento da ação do grupo estrutural $\mathbb{Z}_{2}$ na fibra $Z$, portanto podemos definir $T_{x_{0} x_{1}}^{*} \sim \mathbb{1}, T_{x_{1} x_{2}}^{*} \sim \mathbb{1}$ e $T_{x_{0} x_{2}}^{*} \sim A$, onde $A$ é a aplicação antipodal.

Note que depois de passar pelos homomorfismos $T_{x_{i} x_{i+1}}^{*}$, é como se os coeficientes passassem a ter um caráter global e não mais local - com isso podemos realizar operações com coeficientes sobre fibras "diferentes", fazendo com que sua soma tenha sentido.

Segundo a definição do operador bordo dada acima, segue

- $\partial\left(\alpha_{0}\left(x_{0} x_{1}\right)\right)=T_{x_{0} x_{1}}^{*}\left(\alpha_{0}\right) v_{1}+(-1)^{1} \alpha_{0} v_{0}=\alpha_{0} v_{1}-\alpha_{0} v_{0}$

- $\partial\left(\alpha_{1}\left(x_{1} x_{2}\right)\right)=T_{x_{1} x_{2}}^{*}\left(\alpha_{1}\right) v_{2}+(-1)^{1} \alpha_{1} v_{1}=\alpha_{1} v_{2}-\alpha_{1} v_{1}$

- $\partial\left(\alpha_{2}\left(x_{0} x_{2}\right)\right)=T_{x_{0} x_{2}}^{*}\left(\alpha_{2}\right) v_{2}+(-1)^{1} \alpha_{2} v_{0}=-\alpha_{2} v_{2}-\alpha_{2} v_{0}$

Daí vem $\partial\left(\sum_{i=0}^{2} \alpha_{i}\left(x_{i} x_{i+1}\right)\right)=\sum_{i=0}^{2} \partial\left(\alpha_{i}\left(x_{i} x_{i+1}\right)\right)=\alpha_{0} v_{1}-\alpha_{0} v_{0}+\alpha_{1} v_{2}-\alpha_{1} v_{1}-$ $\alpha_{2} v_{2}-\alpha_{2} v_{0}=\left(-\alpha_{0}-\alpha_{2}\right) v_{0}+\left(\alpha_{0}-\alpha_{1}\right) v_{1}+\left(\alpha_{1}-\alpha_{2}\right) v_{2}$.

É claro que $v_{1}=v_{0}+v_{1}-v_{0}$ e que $v_{2}=v_{0}+v_{2}-v_{0}$ e então temos que

$\partial\left(\sum_{i=0}^{2} \alpha_{i}\left(x_{i} x_{i+1}\right)\right)=\left(-\alpha_{0}-\alpha_{2}\right) v_{0}+\left(\alpha_{0}-\alpha_{1}\right)\left(v_{0}+v_{1}-v_{0}\right)+\left(\alpha_{1}-\alpha_{2}\right)\left(v_{0}+v_{2}-\right.$ $\left.v_{0}\right)=\left(-\alpha_{0}-\alpha_{2}+\alpha_{0}-\alpha_{1}+\alpha_{1}-\alpha_{2}\right) v_{0}+\left(\alpha_{0}-\alpha_{1}\right)\left(v_{1}-v_{0}\right)+\left(\alpha_{1}-\alpha_{2}\right)\left(v_{2}-v_{0}\right)=$ $-\alpha_{2}\left(2 v_{0}\right)+\left(\alpha_{0}-\alpha_{1}\right)\left(v_{1}-v_{0}\right)+\left(\alpha_{1}-\alpha_{2}\right)\left(v_{2}-v_{0}\right)$.

Portanto a imagem de $\partial$ é gerada por $\left\{2 v_{0}, v_{1}-v_{0}, v_{2}-v_{0}\right\}$. Como $C_{0}$ é gerado por $\left\{v_{0}, v_{1}, v_{2}\right\}$ que é um grupo de geradores equivalente a $\left\{v_{0}, v_{1}-v_{0}, v_{2}-v_{0}\right\}$, segue que

$$
H_{0}\left(S^{1}, E\right)=\frac{<v_{0}, v_{1}-v_{0}, v_{2}-v_{0}>}{<2 v_{0}, v_{1}-v_{0}, v_{2}-v_{0}>} \approx \mathbb{Z}_{2}
$$

Agora, como $\partial\left(\sum_{i=0}^{2} \alpha_{i}\left(x_{i} x_{i+1}\right)\right)=-\alpha_{2}\left(2 v_{0}\right)+\left(\alpha_{0}-\alpha_{1}\right)\left(v_{1}-v_{0}\right)+\left(\alpha_{1}-\alpha_{2}\right)\left(v_{2}-\right.$ $\left.v_{0}\right)$, temos que $\partial\left(\sum_{i=0}^{2} \alpha_{i}\left(x_{i} x_{i+1}\right)\right)$ se anula se e só se $-\alpha_{2}=0, \alpha_{0}-\alpha_{1}=0$ e $\alpha_{1}-$ $\alpha_{2}=0$. Mas se isso ocorre, então $\alpha_{0}=\alpha_{1}=\alpha_{2}=0$ e portanto uma 1-cadeia se anula se e só se ela é nula. Daí segue que $H_{1}\left(S^{1}, E\right)=0$.

Agora mostraremos alguns resultados que envolvem homologia com coeficientes locais. O primeiro deles, retirado de [11], é o seguinte diagrama comutativo que tem todas as linhas exatas, de acordo com o que vimos antes nesta mesma seção: 


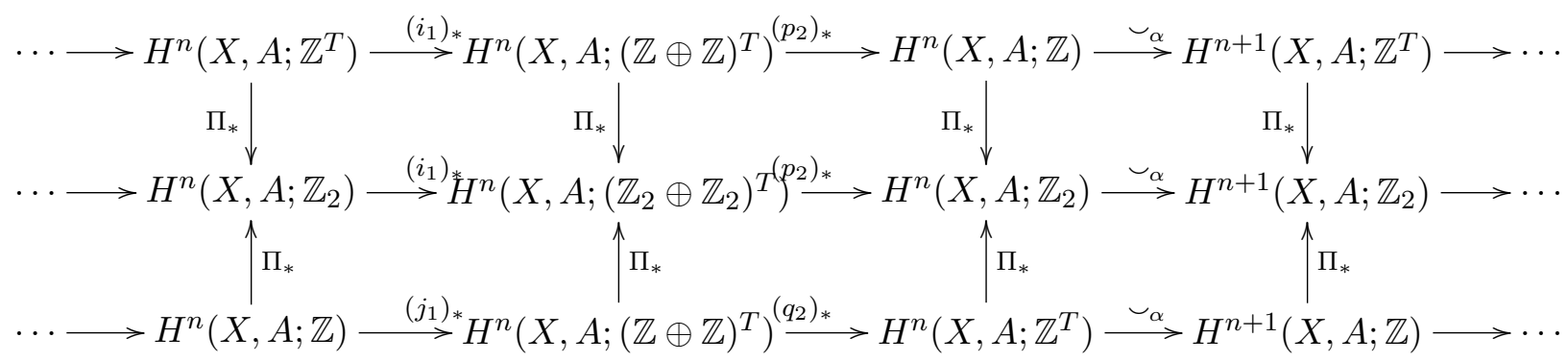

A partir deste diagrama e das conhecidas cohomologias $H^{n}\left(\mathbb{R} P_{k} ; \mathbb{Z}_{2}\right)$ e $H^{n}\left(\mathbb{R} P_{k} ; \mathbb{Z}\right)$ é possível calcular as cohomologias $H^{n}\left(\mathbb{R} P_{k} ; \mathbb{Z}^{T}[u]\right), H^{n}\left(\mathbb{R} P_{k} ;(\mathbb{Z} \oplus \mathbb{Z})^{T}[u]\right)$ e $H^{n}\left(\mathbb{R} P_{k} ;\left(\mathbb{Z}_{2} \oplus \mathbb{Z}_{2}\right)^{T}[u]\right)$

Proposição 16. A cohomologia de $\mathbb{R} P_{k}$ com coeficientes em $\left(\mathbb{Z}_{2} \oplus \mathbb{Z}_{2}\right)^{T}[u]$ é dada por

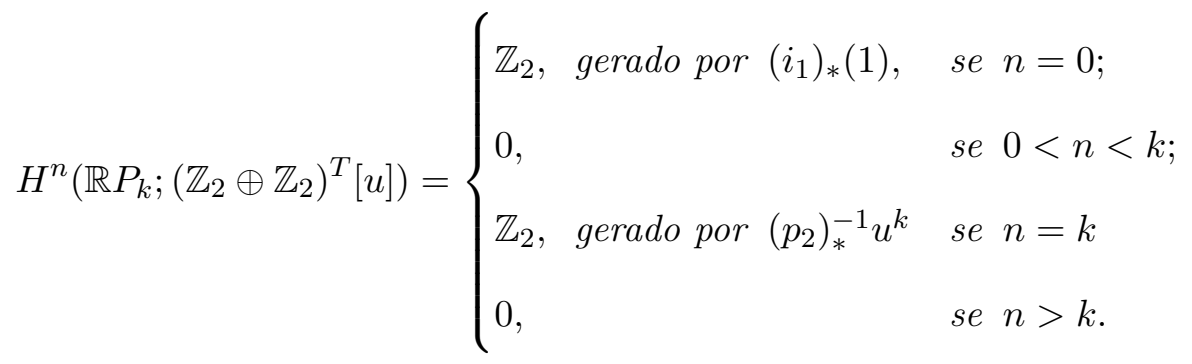

Demonstração.

- Caso $n=0$ : Ainda pelo diagrama 2.2, temos que

$0 \rightarrow H^{0}\left(\mathbb{R} P_{k} ; \mathbb{Z}_{2}\right) \approx \mathbb{Z}_{2} \stackrel{i_{1 *}}{\rightarrow} H^{0}\left(\mathbb{R} P_{k} ;\left(\mathbb{Z}_{2} \oplus \mathbb{Z}_{2}\right)^{T}[u]\right) \stackrel{p_{2 *}}{\rightarrow} H^{0}\left(\mathbb{R} P_{k} ; \mathbb{Z}_{2}\right) \approx \mathbb{Z}_{2} \stackrel{\lrcorner_{\alpha}}{\rightarrow} H^{1}\left(\mathbb{R} P_{k} ; \mathbb{Z}_{2}\right) \approx \mathbb{Z}_{2}$

e como $\smile_{\alpha}$ é um isomorfismo aqui, temos que $p_{2 *}$ é nulo, e daí $i_{1 *}\left(\mathbb{Z}_{2}\right)$ além de injetor é também sobrejetor, e portanto $H^{0}\left(\mathbb{R} P_{k} ;\left(\mathbb{Z}_{2} \oplus \mathbb{Z}_{2}\right)^{T}[u]\right) \approx \mathbb{Z}_{2}$.

- Se $0<n<k$, temos ainda:

$$
\begin{aligned}
H^{n-1}\left(\mathbb{R} P_{k} ; \mathbb{Z}_{2}\right) \approx \mathbb{Z}_{2} \stackrel{\lrcorner_{\alpha}}{\rightarrow} H^{n}\left(\mathbb{R} P_{k} ; \mathbb{Z}_{2}\right) \approx \mathbb{Z}_{2} \stackrel{i_{1_{*}}}{\rightarrow} H^{n}\left(\mathbb{R} P_{k} ;\left(\mathbb{Z}_{2} \oplus \mathbb{Z}_{2}\right)^{T}[u]\right) \stackrel{p_{2_{*}}}{\longrightarrow} \\
\stackrel{p_{2_{*}}}{\longrightarrow} H^{n}\left(\mathbb{R} P_{k} ; \mathbb{Z}_{2}\right) \approx \mathbb{Z}_{2} \stackrel{\lrcorner_{\alpha}}{\rightarrow} H^{n+1}\left(\mathbb{R} P_{k} ; \mathbb{Z}_{2}\right) \approx \mathbb{Z}_{2}
\end{aligned}
$$

onde $\smile_{\alpha}$ é sempre isomorfismo. Daí segue que $H^{n}\left(\mathbb{R} P_{k} ;\left(\mathbb{Z}_{2} \oplus \mathbb{Z}_{2}\right)^{T}[u]\right)$ é necessariamente zero. 
- Para $n=k$ temos

$$
\begin{gathered}
H^{n-1}\left(\mathbb{R} P_{k} ; \mathbb{Z}_{2}\right) \approx \mathbb{Z}_{2} \stackrel{\lrcorner_{\alpha}}{\rightarrow} H^{n}\left(\mathbb{R} P_{k} ; \mathbb{Z}_{2}\right) \approx \mathbb{Z}_{2} \stackrel{i_{1_{*}}}{\rightarrow} H^{n}\left(\mathbb{R} P_{k} ;\left(\mathbb{Z}_{2} \oplus \mathbb{Z}_{2}\right)^{T}[u]\right) \stackrel{p_{2_{*}}}{\longrightarrow} \\
\stackrel{p_{2_{*}}}{\longrightarrow} H^{n}\left(\mathbb{R} P_{k} ; \mathbb{Z}_{2}\right) \approx \mathbb{Z}_{2} \stackrel{\smile_{\alpha}}{\longrightarrow} H^{n+1}\left(\mathbb{R} P_{k} ; \mathbb{Z}_{2}\right) \approx 0
\end{gathered}
$$

Onde o primeiro $\smile_{\alpha}$ é um isomorfismo e daí $H^{n}\left(\mathbb{R} P_{k} ;\left(\mathbb{Z}_{2} \oplus \mathbb{Z}_{2}\right)^{T}[u]\right) \approx \mathbb{Z}_{2}$.

- Para o último caso $n>k$ a situação é a mais simples possível:

$$
0 \rightarrow H^{n}\left(\mathbb{R} P_{k} ;\left(\mathbb{Z}_{2} \oplus \mathbb{Z}_{2}\right)^{T}[u]\right) \rightarrow 0,
$$

de onde claramente $H^{n}\left(\mathbb{R} P_{k} ;\left(\mathbb{Z}_{2} \oplus \mathbb{Z}_{2}\right)^{T}[u]\right) \approx 0$.

Proposição 17. A homologia de $\mathbb{R} P_{k}$ com coeficientes em $\mathbb{Z}^{T}[u]$ e em $(\mathbb{Z} \oplus \mathbb{Z})^{T}[u]$ e em é dada por

$H^{n}\left(\mathbb{R} P_{k} ; \mathbb{Z}^{T}[u]\right) \approx \begin{cases}\mathbb{Z}_{2}, \text { gerado por } \bar{u}^{n}, & \text { se } n \text { é impar com } 0<n \leq k ; \\ 0 & \text { se } n \text { é par, } 0<n<k ; \\ \mathbb{Z}, \text { gerado pela classe top } t\left(\mathbb{R} P_{k}\right) & \text { se } n=k \text { par } ; \\ 0, & \text { se } n>k .\end{cases}$

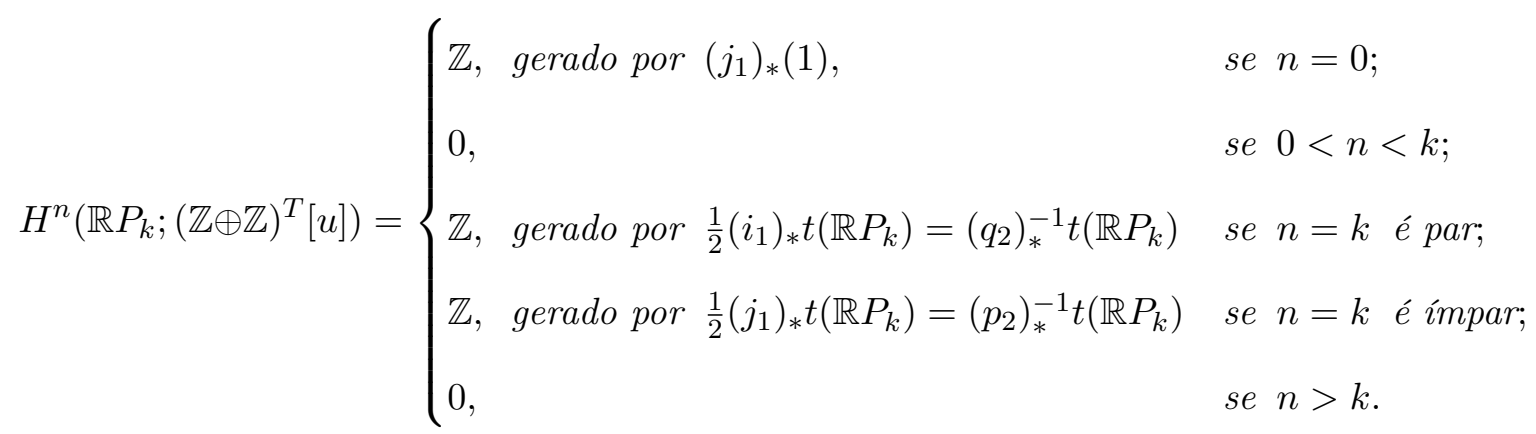

Demonstração. Os diagramas para as cohomologias que estamos calculando sobre os sheaves $\mathbb{Z}^{T}$ e $(\mathbb{Z} \oplus \mathbb{Z})^{T}$ são interdependentes e para proceder de forma a completar todos os valores, devemos efetuar os cálculos sempre relativamente ao diagrama 2.2 e na seguinte ordem: 
Primeiramente notemos que para $n>k$ as projeções de $H^{n}\left(\mathbb{R} P^{k} ; \mathbb{Z}^{T}\right)$ e $H^{n}\left(\mathbb{R} P^{k} ;(\mathbb{Z} \oplus\right.$ $\mathbb{Z})^{T}$ ) por $\pi_{*}$ sempre são nulas, o que indica que não há nenhum elemento não trivial nestes grupos. Daí $H^{n}\left(\mathbb{R} P^{k} ; \mathbb{Z}^{T}\right)=H^{n}\left(\mathbb{R} P^{k} ;(\mathbb{Z} \oplus \mathbb{Z})^{T}\right)=0$ para $n>k$.

Verifiquemos agora a estrutura de $H^{n}\left(\mathbb{R} P^{k} ; \mathbb{Z}^{T}\right)$ quando $0 \leq n<k$ é par:

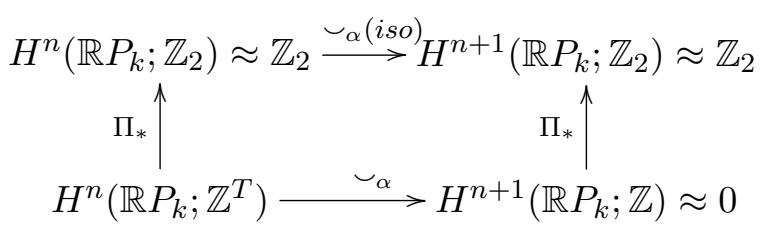

Note que neste caso a comutatividade do diagrama nos leva a concluir que ou $\Pi_{*}$ partindo de $H^{n}\left(\mathbb{R} P_{k} ; \mathbb{Z}^{T}\right)$ é nula ou $H^{n}\left(\mathbb{R} P_{k} ; \mathbb{Z}^{T}\right)=0$. Uma vez que se houver classe não nula em $H^{n}\left(\mathbb{R} P_{k} ; \mathbb{Z}^{T}\right)$ é possível tomarmos um coeficiente para essa classe de tal forma que sua imagem por $\pi_{*}$ não se anule, segue que só podemos ter $H^{n}\left(\mathbb{R} P_{k} ; \mathbb{Z}^{T}\right)=$ 0 .

O passo seguinte é verificar $H^{n}\left(\mathbb{R} P^{k} ;(\mathbb{Z} \oplus \mathbb{Z})^{T}\right)$ quando $n=0$. Para isso vejamos o diagrama abaixo, retirado daquele de 2.2 :

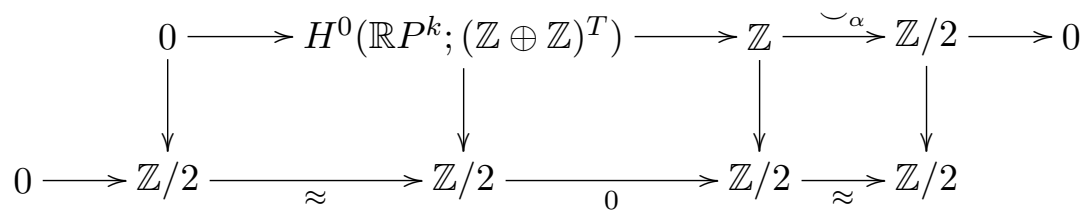

Como o diagrama comuta, então $\smile_{\alpha}$ só pode ser sobrejetora e assim a sequïência exata curta da primeira linha nos garante que $H^{0}\left(\mathbb{R} P^{k} ;(\mathbb{Z} \oplus \mathbb{Z})^{T}\right)=2 \mathbb{Z} \approx \mathbb{Z}$.

Agora passemos ao mesmo $H^{0}\left(\mathbb{R} P^{k} ;(\mathbb{Z} \oplus \mathbb{Z})^{T}\right)$, mas agora com o diferencial de que $0<n<k$ é par:

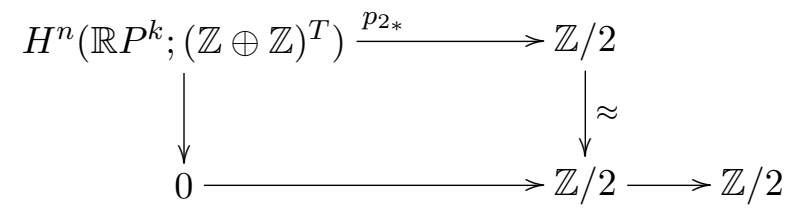

Se $H^{n}\left(\mathbb{R} P^{k} ;(\mathbb{Z} \oplus \mathbb{Z})^{T}\right)$ é não nulo, é possível então escolher coeficientes de tal forma que a imagem de um gerador por $p_{2_{*}}$ não se anule, o que faria o diagrama não comutar, o que é absurdo. Segue daí que $H^{n}\left(\mathbb{R} P^{k} ;(\mathbb{Z} \oplus \mathbb{Z})^{T}\right)=0$ se $0<n<k$ é par.

Agora vejamos esta outra parte do diagrama tomado para $0<n<k$ ímpar: 


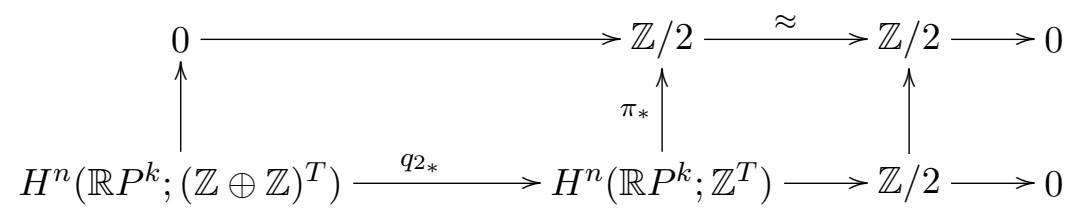

Primeiramente notemos que $H^{n}\left(\mathbb{R} P^{k} ; \mathbb{Z}^{T}\right)$ não pode ser nulo porque a seqüência é exata. Agora suponhamos que $H^{n}\left(\mathbb{R} P^{k} ;(\mathbb{Z} \oplus \mathbb{Z})^{T}\right)$ tenha um elemento não trivial. Então seria possível fazer uma escolha de coeficientes de tal forma que $q_{2_{*}}$ não se anule - o mesmo ocorrendo com $\pi_{*}$ - e isso faria o diagrama não ser comutativo. Assim, temos $H^{n}\left(\mathbb{R} P^{k} ;(\mathbb{Z} \oplus \mathbb{Z})^{T}\right)=0$ e mais ainda, disso segue que $H^{n}\left(\mathbb{R} P^{k} ; \mathbb{Z}^{T}\right)=\mathbb{Z} / 2$, sempre para $0<n<k$ ímpar.

Passemos então para o caso onde $k=n$ par. O diagrama é o seguinte:

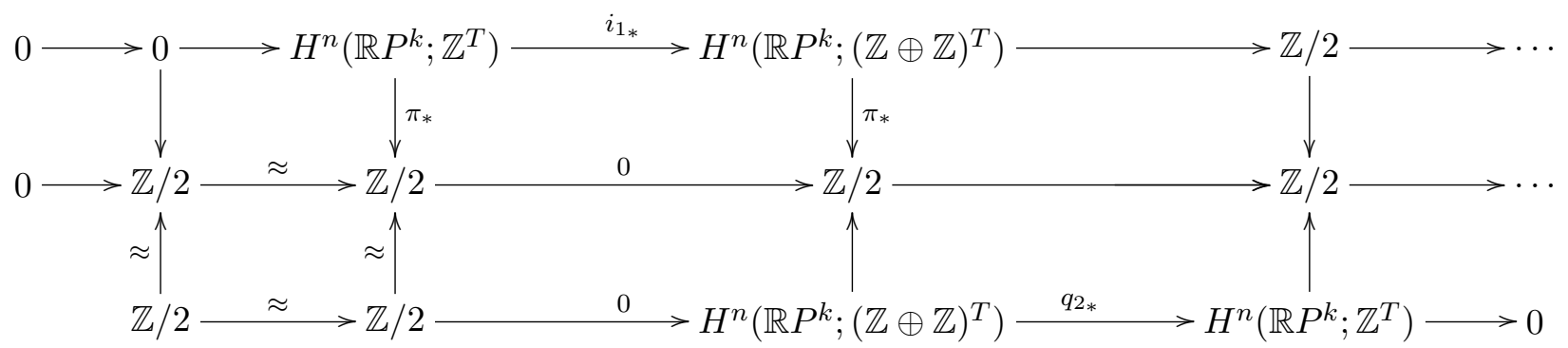

Aqui podemos perceber que a última linha nos fornece um isomorfismo entre $H^{n}\left(\mathbb{R} P^{k} ; \mathbb{Z}^{T}\right)$ e $H^{n}\left(\mathbb{R} P^{k} ;(\mathbb{Z} \oplus \mathbb{Z})^{T}\right)$ enquanto que suas projeções não triviais pelas aplicações $\pi_{*}$ garantem que tais grupos não são nulos. A primeira linha também oferece a importante informação de que tais grupos não são de torção, uma vez que se fossem, a aplicação $i_{1_{*}}$, que é uma injeção, seria também um isomorfismo, o que faria com que a exatidão não procedesse. Assim, $H^{n}\left(\mathbb{R} P^{k} ; \mathbb{Z}^{T}\right)$ e $H^{n}\left(\mathbb{R} P^{k} ;(\mathbb{Z} \oplus \mathbb{Z})^{T}\right)$ só podem ser somas diretas cujos somandos são isomorfos a $\mathbb{Z}$. Como a projeção $\pi_{*}$ aponta para $\mathbb{Z} / 2$, segue que tais grupos são, na verdade, $\mathbb{Z}$ quando $k=n$ é par.

O último caso é exatamente o de $k=n$ ímpar e para tal temos o seguinte diagrama:

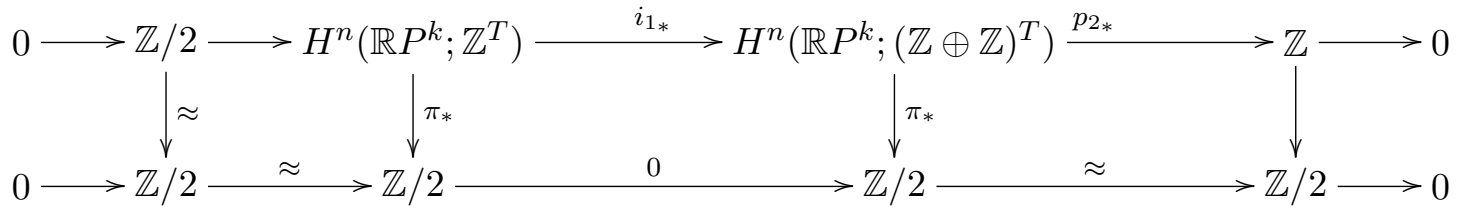

Primeiro observemos que $H^{n}\left(\mathbb{R} P^{k} ;(\mathbb{Z} \oplus \mathbb{Z})^{T}\right)$ não é nulo porque a seqüência é exata. Notemos ainda que $\operatorname{ker}\left(i_{1_{*}}\right)=\mathbb{Z} / 2$ e suponhamos que exista um elemento $a \in H^{n}\left(\mathbb{R} P^{k} ; \mathbb{Z}^{T}\right)$ que não esteja no kernel de $i_{1_{*}}$. Seria possível então se fazer uma escolha de coeficientes de tal forma que $i_{1_{*}}(a)$ tivesse imagem não nula por $\pi_{*}$. Agora, 
como as linhas são exatas, a imagem deste mesmo $i_{1_{*}}(a)$ por $p_{2_{*}}$ seria nula, o que faria o diagrama não comutar, o que é absurdo. Segue daí que $H^{n}\left(\mathbb{R} P^{k} ; \mathbb{Z}^{T}\right)=\mathbb{Z} / 2$ e a partir disso $H^{n}\left(\mathbb{R} P^{k} ;(\mathbb{Z} \oplus \mathbb{Z})^{T}\right)=\mathbb{Z}$ sempre que $n=k$ é ímpar.

\subsection{Resoluções}

Através das resoluções será possível calcular a homologia e a cohomologia das spherical space forms. Esta seção tem por objetivo fazer uma breve introdução ao estudo das resoluções, em particular daquelas conhecidas por resoluções livres.

Definição 2.9. Seja $R$ um anel e $M$ um $R$-módulo à esquerda. Então uma resolução de $M$ é uma seqüência exata de $R$-módulos

$$
\cdots \rightarrow F_{1} \rightarrow F_{0} \stackrel{\epsilon}{\rightarrow} M \rightarrow 0
$$

onde a aplicação $\epsilon$ é chamada de aumentação e, se cada $F_{i}$ é livre, a resolução é chamada de resolução livre.

Observação 2.2. Uma resolução pode ser vista como um complexo de cadeias no qual o termo correspondente à posição - 1 é $M$ - este complexo de cadeias é chamado de complexo de cadeias aumentado associado à resolução.

Exemplo 2.3. Um exemplo bastante simples de resolução para o $\mathbb{Z}$-módulo $\mathbb{Z} / 2$ é dado por $0 \rightarrow \mathbb{Z} \stackrel{2}{\rightarrow} \mathbb{Z} \stackrel{\pi}{\rightarrow} \mathbb{Z} / 2 \rightarrow 0$

Dado um $R$-módulo $M$ existem diversas resoluções livres diferentes para este mesmo $M$, porém a escolha de uma resolução em particular não influencia o cálculo de homologias e cohomologias, como pode ser conferido em [1].

Definição 2.10. Um G-complexo é um par $(X, \varphi)$ onde $X$ é um $C W$-complexo e $\varphi$ é uma ação de $G$ em $X$ que permuta as células deste último.

Dado um $G$-complexo $X$, a ação de $G$ em $X$ induz uma ação de $G$ no complexo de cadeias celular $C_{*}(X)$ e a partir daí temos um complexo de cadeias de $G$-módulos.

Definição 2.11. Um G-complexo $X$ é dito livre se a ação de $G$ em $X$ permuta células livremente, ou seja: $g \sigma \neq \sigma$ se $g \neq 1$.

Em um $G$-complexo livre $X$, cada módulo de cadeias $C_{n}(X)$ é um $\mathbb{Z} G$-módulo livre com um elemento base para cada $G$-órbita de células. A prova desta afirmação, das proposições a seguir e maiores detalhes sobre este tema podem ser encontrados em [1] e também em [13]. 
Proposição 18. Se $X$ é um G-complexo livre e contrátil, então o complexo de cadeias celular aumentado de $X$ é uma resolução livre de $\mathbb{Z}$ sobre $\mathbb{Z} G$.

Proposição 19. Se $X$ é um $K(G, 1)$ então o complexo de cadeias celular aumentado da cobertura universal de $X$ é uma resolução livre de $\mathbb{Z}$ sobre $\mathbb{Z} G$ do tipo

$$
\ldots \rightarrow C_{n}(\tilde{X}) \stackrel{\partial_{n}}{\longrightarrow} C_{n-1}(\tilde{X}) \rightarrow \ldots \rightarrow C_{0}(\tilde{X}) \stackrel{\epsilon}{\rightarrow} \mathbb{Z} \rightarrow 0 .
$$

Veremos nos capítulos seguintes que as spherical space forms tridimensionais são espaços de Eilenberg-MacLane do tipo $K(G, 1)$, onde $G$ é um subgrupo finito de $\mathrm{SO}_{4}$ que age sem pontos fixos (livremente) na esfera $S^{3}$. Assim, para uma spherical space form $S_{\Gamma}$, temos que $\pi_{n}\left(S_{\Gamma}\right)=0$ para $n \geq 2$, o que é equivalente a dizer que a cobertura universal de tais espaços é contrátil.

Definição 2.12. Se $G$ é um grupo e $M$ é um $G$-módulo, o grupo $M_{G}$ de coinvariantes de $M$ é o quociente de $M$ pelo subgrupo aditivo gerado pelos elementos da forma $g m-m, g \in G, m \in M$. Se $F$ é um $\mathbb{Z} G$-módulo livre com base $\left\{e_{i}\right\}$ então $F_{G}$ é um $\mathbb{Z}$-módulo livre com base $\left\{\overline{e_{i}}\right\}$.

Proposição 20. Sejam $X$ um $G$-complexo livre e $Y=X / G$ o espaço das órbitas. Então $C_{*}(Y)=C_{*}(X)_{G}$.

A homologia de um grupo $G$ pode então ser definida assim:

Definição 2.13. Dados um grupo $G$ e uma resolução livre $\epsilon: F \rightarrow \mathbb{Z}$ de $\mathbb{Z}$ sobre $\mathbb{Z} G$, definimos os grupos de homologia de $G$ por $H_{i}(G)=H_{i}\left(F_{G}\right)$.

É possível se provar também que a menos de homotopia, $F_{G}$ não depende da resolução escolhida e portanto nossa definição de homologia para o grupo $G$ é independente da resolução.

Proposição 21. Se $Y$ é um $K(G, 1)$-complexo então $H_{*}(G)=H_{*}(Y)$.

O resultado anterior nos permite calcular a homologia das "spherical space forms" a partir da homologia definida para um grupo $G$, que por sua vez é obtida a partir de uma resolução para $G$. Vamos agora estudar resoluções específicas para determinados grupos.

Seja $G$ um grupo finito com apresentação $0 \rightarrow R \rightarrow F \stackrel{p}{\rightarrow} G \rightarrow 0$, onde $F$ e $R$ são livres nos conjuntos $S$ e $T$, respectivamente. Então de acordo com [5], temos a seguinte resolução de $\mathbb{Z}$ sobre $\mathbb{Z} G$ :

$$
\cdots \longrightarrow f w / f w^{2} \longrightarrow w / w^{2} \longrightarrow f / f w \underset{p^{\prime}}{\longrightarrow} \mathbb{Z} G \underset{\epsilon}{\longrightarrow} \longrightarrow 0,
$$


onde $\varepsilon: \mathbb{Z} G \rightarrow \mathbb{Z}$ é a aplicação de aumentação (augmentation), $f$ é o ideal de aumentação de $F, w$ é o kernel de $\mathbb{Z} p: \mathbb{Z} F \rightarrow \mathbb{Z} G, p^{\prime}$ é induzida por $p$ e todos os outros homomorfismos são induzidos pelas devidas inclusões.

Proposição 22. Para qualquer $G$ como descrito acima, temos a seguinte resolução de $\mathbb{Z}$ sobre $\mathbb{Z} G$ :

$$
\cdots \longrightarrow \mathbb{Z} G[C] \underset{i}{\longrightarrow} \mathbb{Z} G[B] \underset{\phi_{2}}{\longrightarrow} \mathbb{Z} G[A] \underset{\phi_{1}}{\longrightarrow} \mathbb{Z} G[e] \underset{\epsilon}{\longrightarrow} \longrightarrow 0,
$$

onde $A$ e $B$ denotam conjuntos ordenados de geradores abstratos dos módulos (um gerador para cada elemento no conjunto correspondente $S$ ou $T$ ), "e" é um gerador abstrato, $C$ é o subconjunto de $B$ dos geradores do $\mathbb{Z} G$-submódulo não livre $k e r \phi_{2}$, " $i$ " é o homomorfismo induzido pela inclusão, e os outros homomorfismos são definidos nos elementos da base da seguinte forma:

$$
\begin{gathered}
\phi_{1}(a)=\left(1-s_{a}\right) e \\
\phi_{2}(b)=\sum_{a \in A}\left(d_{s_{b}} r_{a}\right) a,
\end{gathered}
$$

e se extendem por linearidade. Aqui, $s_{b}$ e $r_{a}$ denotam os elementos no conjunto dos geradores do grupo que correspondem aos elementos abstratos da base, e a derivação é definida nos elementos de $F$ segundo descrito em [1]: $d_{s} 1=0, d_{s}(u v)=d_{s}(u)+u d_{s} v$, para cada $s \in S$.

Demonstração. Começamos com a resolução livre de $\mathbb{Z}$ sobre $\mathbb{Z} G$ dada por Gruenberg em [5]:

$$
\cdots \longrightarrow \mathbb{Z} G[W] \underset{\phi_{3}}{\longrightarrow} \mathbb{Z} G[X] \underset{\phi_{2}}{\longrightarrow} \mathbb{Z} G[Y] \underset{\phi_{1}}{\longrightarrow} \mathbb{Z} G[e] \underset{\epsilon}{\longrightarrow} \longrightarrow 0,
$$

onde $W$ e um conjunto finito de geradores. Agora suponha que

$$
\cdots \longrightarrow K \underset{f}{\longrightarrow} L \underset{g}{\longrightarrow} M \underset{h}{\longrightarrow} N \underset{\epsilon}{\longrightarrow} \mathbb{Z} \longrightarrow 0,
$$

é uma resolução livre de $\mathbb{Z}$ sobre um anel $R$, com módulos finitamente gerados $K, L, M$ e $N$ satisfazendo a propriedade de que, duas a duas, as suas bases tem a mesma cardinalidade. O módulo $H=\operatorname{ker}(g: L \rightarrow M)$ aparece na seqüência exata

$$
0 \longrightarrow H \underset{i}{\longrightarrow} L \underset{g}{\longrightarrow} M
$$

E por outro lado, $K$ é o quociente de um módulo livre finitamente gerado $P$ por algum submódulo $Q$, e então temos a seguinte seqüência exata 


$$
Q \underset{j}{\longrightarrow} P \underset{p}{\longrightarrow} H \longrightarrow 0,
$$

podemos então aplicar o splicing lema para obter a seqüência exata

$$
Q \underset{j}{\longrightarrow} P \underset{i p}{\longrightarrow} L \underset{g}{\longrightarrow} M \underset{h}{\longrightarrow} N \underset{\epsilon}{\longrightarrow} \mathbb{Z} \longrightarrow 0,
$$

onde $P, L, M$, e $N$ são livres. Aplicando este processo à resolução acima com $H=\operatorname{ker} \phi_{2}$ é suficiente para terminarmos a prova. 


\section{Capítulo 3}

\section{Torres de Postnikov e seqüências}

\section{espectrais}

Este capítulo trata de duas ferramentas relativamente avançadas da topologia algébrica que foram largamente utilizadas durante todo este trabalho, as torres (ou sistemas) de Postnikov e as seqüências espectrais.

Para mostrar a importância das torres de Postnikov para nós, precisamos da seguinte proposição, que não é trivial e cuja prova pode ser encontrada em [25]:

Proposição 23. Se a dimensão de $X$ é $m$ então $[X, Y]$ - o conjunto de classes de homotopia de aplicações de $X$ para $Y$ - tem na função $[f] \mapsto\left[P_{m} f\right]$ uma bijeção com o conjunto $\left[X, Y_{m}\right]$, onde $Y_{m}$ é o m-ésimo nivel da torre de Postnikov de $Y$.

Esta proposição, juntamente com o teorema 1.1 da classificação dos fibrados tornam possível a enumeração de fibrados uma vez que tenhamos a descrição do conjunto $\left[X, Y_{m}\right]$ e é justamente na obtenção deste último que se faz necessária a utilização da torre de Posnikov.

A decomposição de um CW-complexo em uma torre de Postnikov é dual da decomposição deste CW-complexo em seu esqueleto. Os blocos utilizados para se construir um CW-complexo são células $\left(D^{n}, S^{n-1}\right)$, que tem homologia $\mathbb{Z}$ na dimensão $n$ e zero nas outras dimensões e no caso das torres de Postnikov, os blocos utilizados são espaços de Eilenberg-McLane do tipo $k(\pi, n)$. Mais ainda, nos CW-complexos as aplicações de colagem descrevem como as células são coladas enquanto nas torres de Postnikov os espaços são descritos como fibrações iteradas cujas fibras são espaços de Eilenberg-McLane e a obstrução primária a se encontrar um cross-section determina como os espaços são colados a partir de seus $k(\pi, n)$. 
Cada fibração $p_{n}: X_{n} \rightarrow X_{n-1}$ na torre de Postnikov para um espaço $X$ tem fibra $K\left(\pi_{n}(X), n\right)$, e portanto existe uma única obstrução (a obstrução primária) a encontrar um cross-section, que está exatamente em

$$
H^{n+1}\left(X_{n-1} ; \pi_{n}\left(K\left(\pi_{n}(X), n\right)\right)\right)=H^{n+1}\left(X_{n-1}, \pi_{n} X\right)
$$

e é também chamada de $(n+1)$-ésimo $k$-invariante de $X$, denotado por $k^{n+1}$. É importante observar que apenas os grupos de homotopia geralmente não são suficientes para determinar o tipo de homotopia de um CW-complexo. Tal tipo de homotopia é determinado por estes grupos juntamente com os respectivos $k$-invariantes.

Assim, as torres de Postnikov associam a um espaço (simplesmente conexo) conexo por caminhos $X$ uma coleção $\left\{\pi_{n}, p_{n}, k^{n}\right\}$ tal que:

1. $\pi_{n}$ é um grupo abeliano;

2. $p_{n}: X_{n} \rightarrow X_{n-1}$ é uma fibração com fibra $K\left(\pi_{n}, n\right)$;

3. $X_{0}$ é contráctil;

4. $k^{n} \in H^{n}\left(X_{n-2}, \pi_{n-1}\right)$ classifica $p_{n-1}$;

5. A inclusão das fibras induz um isomorfismo

$$
\pi_{n}\left(K\left(\pi_{n}, n\right)\right) \rightarrow \pi_{n}\left(X_{n}\right)
$$

Mais especificamente, temos a seguinte

Definição 3.1. Seja $X$ um espaço topológico conexo. De acordo com [6], uma torre de Postnikov para $X$ é um objeto construído da seguinte maneira: Para todo $n \geq 0$ temos um espaço topológico $X_{n}$ tal que $\pi_{m}\left(X_{n}\right)=0 \quad \forall m>n$ e também uma aplicação $p_{n}: X \rightarrow X_{n}$ que induz isomorfismo nos grupos de homotopia de indice menor ou igual a $n$. Mais ainda, para todo $n \geq 1$, temos também uma fibração $q_{n}: X_{n} \rightarrow X_{n-1}$ tal que $p_{n-1}=q_{n} p_{n}$. 


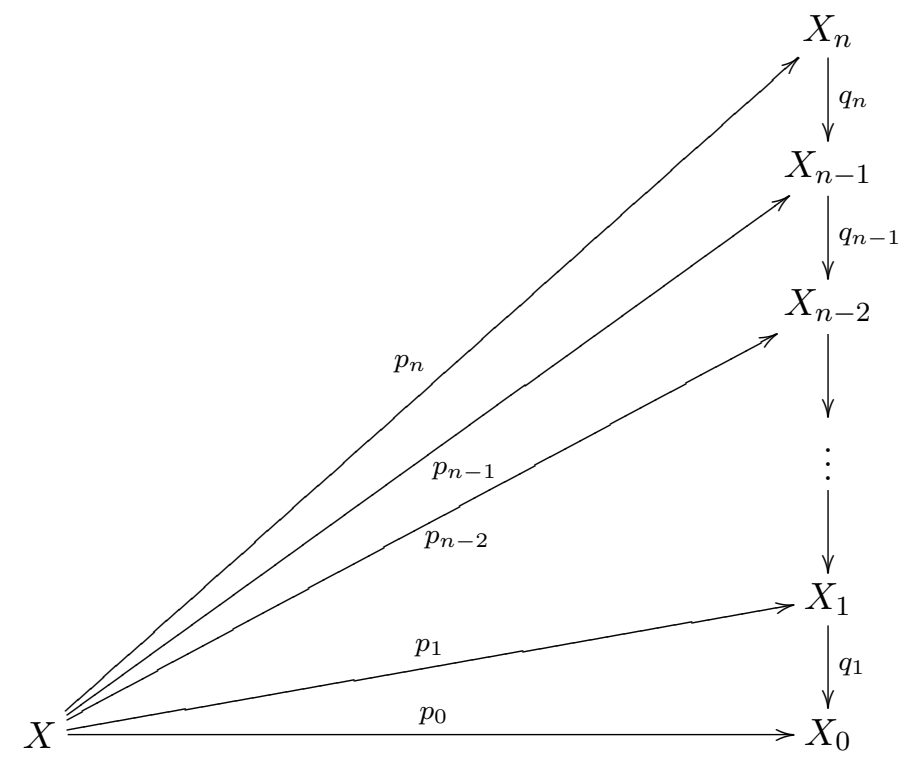

O diagrama acima ilustra uma torre de Postnikov para um espaço topológico conexo $X$.

Proposição 24. A fibra de cada $q_{n}$ é um espaço de Eilenberg-MacLane do tipo $K\left(\pi_{n}(X), n\right)$.

Demonstração. Para ver isto, recorremos à seguinte sequência de homotopia da fibração $q_{n}$, onde $F$ é a notação para a fibra $q_{n}^{-1}(y) \subset X_{n}$, com $y \in X_{n-1}$ :

$\cdots \rightarrow \pi_{i+1}(F) \rightarrow \pi_{i+1}\left(X_{n}\right) \rightarrow \pi_{i+1}\left(X_{n-1}\right) \rightarrow \pi_{i}(F) \rightarrow \pi_{i}\left(X_{n}\right) \rightarrow \pi_{i}\left(X_{n-1}\right) \rightarrow \ldots$

Quando $i>n$ ela se resume a $0 \rightarrow \pi_{i}(F) \rightarrow 0$ e portanto $\pi_{i}(F)=0$ para $i>n$.

Quando $i=n$, temos $0 \rightarrow \pi_{n}(F) \rightarrow \pi_{n}\left(X_{n}\right) \rightarrow 0$ e portanto é claro que $\pi_{n}(F) \approx$ $\pi_{n}\left(X_{n}\right)$.

Para o caso $i=k<n$ sabemos que todo $\pi_{i}\left(X_{k}\right)$ é isomorfo a $\pi_{i}(X)$ por $p_{k}$, e isso nos leva a concluir que existe isomorfismo $\pi_{i+1}\left(X_{n}\right) \approx \pi_{i+1}\left(X_{n-1}\right)$. Um argumento idêntico nos leva a um isomorfismo entre $\pi_{i}\left(X_{n}\right)$ e $\pi_{i}\left(X_{n-1}\right)$. Logo temos uma sequência $0 \rightarrow \pi_{n}(F) \rightarrow 0$, de onde $\pi_{n}(F) \approx 0$.

O principal resultado sobre as torres de Postnikov é o seguinte:

Teorema 3.1. O tipo de homotopia fraco de $X$ é determinado por sua torre de Postnikov. Mais precisamente, dada uma terna $\pi_{n}, p_{n}, k^{n}$ satisfazendo as condições 1 - 5 acima, existe um espaço $X$ com esta torre de Postnikov. Se $Y$ é qualquer outro espaço com esta mesma decomposição de Postnikov, então $X$ e $Y$ tem o mesmo tipo de homotopia fraca. 


\subsection{Seqüências espectrais}

As seqüências espectrais são as ferramentas pelas quais obtemos explicitamente os fibrados que são nosso objeto de estudo. Esta seção pretende introduzir tais seqüências, seus elementos e algumas de suas propriedades. O método aqui escolhido para a construção de tal objeto é o dos pares exatos, justamente porque L. L. Larmore assim escolheu em [11].

Definição 3.2. Seja A um grupo abeliano. Um endomorfismo $d: A \rightarrow A$ é um operador diferencial em $A$ se $d(d(a))=0 \forall a \in A$. Um grupo abeliano com um dado operador diferencial é chamado grupo diferencial ou d-grupo. Se $f$ é um homomorfismo entre dois d-grupos $A$ e $B$ que comuta com d, então dizemos que $f$ é um homomorfismo de d-grupos.

Se temos $f: A \rightarrow B$ como descrito acima e mais, $A \subset B$ e ainda $f(a)=a \forall a \in$ $A$, então dizemos que $A$ é subgrupo do $d$-grupo $B$.

Seja $C=\frac{B}{A}$. Então é possível definir um operador diferencial em $C$ de tal forma que a projeção $g: B \rightarrow C$ seja um homomorfismo de $d$-grupos. Com este operador diferencial, $C$ é chamado $d$-grupo quociente. Assim, temos uma sequência exata $0 \rightarrow A \stackrel{f}{\rightarrow} B \stackrel{g}{\rightarrow} C \rightarrow 0$, e a todo $d$-grupo quociente corresponde uma sequência como esta.

Definição 3.3. Dado d-grupo A, o subgrupo ker(d), chamado de grupo dos ciclos de $A$, será denotado por $\mathfrak{L}(A)$ (com operador diferencial trivial), e o subgrupo $\operatorname{Im}(d)$, chamado de grupo dos bordos de A (com operador diferencial trivial), será denotado por $\mathfrak{B}(A)$. Note que como $d d=0$, então $\mathfrak{B}(A) \subset \mathfrak{L}(A)$, e portanto faz sentido falarmos no d-grupo quociente $\mathfrak{H}(A)=\frac{\mathfrak{L}(A)}{\mathfrak{B}(A)}$ (com operador diferencial trivial), que é chamado de grupo derivado do d-grupo A.

Note que se $f: A \rightarrow B$ é um homomorfismo de $d$-grupos, então $d f=f d$ implica que $\mathfrak{L}(A)$ é levado de maneira injetiva em $\mathfrak{L}(B)$ e $\mathfrak{B}(A)$ é levado de maneira injetiva em $\mathfrak{B}(B)$, o que nos leva a concluir que $f$ induz uma aplicação $\mathfrak{H}(f): \mathfrak{H}(A) \rightarrow \mathfrak{H}(B)$. Para maiores detalhes, confira [6].

Definição 3.4. Um grupo abeliano $A$ é dito grupo graduado se para todo $n \in \mathbb{Z}$ existe um subgrupo $A_{n} \subset A$ tal que $A=\sum_{n} A_{n}$. Os elementos do subgrupo $A_{n}$ são ditos homogênios de grau $\mathbf{n}$.

Similarmente, um grupo abeliano A é dito grupo bi-graduado se para cada par ordenado $(m, n)$ de inteiros existe um subgrupo $A_{m, n} \subset A$ tal que $A=\sum_{m, n} A_{m, n}$. Os elementos do subgrupo $A_{m, n}$ são ditos homogênios de grau $(\mathbf{m}, \mathbf{n})$. 
Observação 3.1. Se $A=\sum_{m, n} A_{m, n}$ e $B=\sum_{m, n} B_{m, n}$ são grupos bi-graduados, então $f: A \rightarrow B$ é dito homogêneo de grau $(p, q)$ se $f\left(A_{m, n}\right) \subset B_{m+p, n+q}, \forall(m, n), m, n \in$ $\mathbb{Z}$.

Definição 3.5. Um par exato é um sistema $\mathfrak{C}=<D, E, i, j, k>$ onde $D, E$ são grupos abelianos e $i: D \rightarrow D, j: D \rightarrow E$ e $k: E \rightarrow D$ são três homomorfismos tais que o seguinte triângulo é exato:

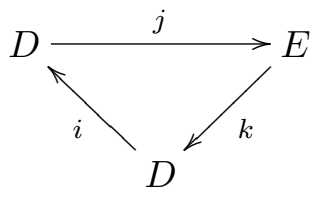

Observação 3.2. Num par exato bi-graduado $\mathfrak{C}=<D, E, i, j, k>$ onde $D$ e E são bi-graduados, então $D^{n}$ e $E^{n}$ também são bigraduados:

$$
D^{n}=\sum_{p, q} D_{p, q}^{n} \quad \text { e } \quad E^{n}=\sum_{p, q} E_{p, q}^{n}
$$

onde $p$ é o grau primário, $q$ é o grau complementar e $p+q$ é o grau total.

Propriedades:

- $\operatorname{deg}\left(i^{n}\right)=\operatorname{deg}(i)$;

- $\operatorname{deg}\left(k^{n}\right)=\operatorname{deg}(k)$;

- $\operatorname{deg}\left(j^{n}\right)=\operatorname{deg}(j)-(n-1)[\operatorname{deg}(i)]$;

- $\operatorname{deg}\left(d^{n}\right)=\operatorname{deg}(j)+\operatorname{deg}(k)-(n-1)[\operatorname{deg}(i)]$.

A partir do par exato $\mathfrak{C}=<D, E, i, j, k>$ podemos construir outro par exato $\mathfrak{C}^{\prime}$ chamado de par derivado. Esta construção do par derivado mostrada a seguir é um detalhamento daquela vista em [6].

Defina o endomorfismo $d: E \rightarrow E$ por $d=j k$. Como pela exatidão temos $k j=0$, segue que $d d=j k j k=j(k j) k=0$ e portanto $d$ é um operador diferencial em $E$.

Seja $D^{\prime}=i(D)$ e $E^{\prime}=\mathfrak{H}(E)$; então $D^{\prime}$ é subgrupo de $D$ e $E^{\prime}$ é subgrupo derivado do grupo $E$. Como $D^{\prime} \subset D$ e $i\left(D^{\prime}\right) \subset D^{\prime}$, podemos definir $i^{\prime}: D^{\prime} \rightarrow D^{\prime}$ por $i^{\prime}=i \mid D^{\prime}$. Segue daí que $k(\mathfrak{L}(E)) \subset D^{\prime}$ porque como $d=j k: E \rightarrow E$, se $x \in \mathfrak{L}(E)$, então $d(x)=j k(x)=0$, de onde $k(x) \in \operatorname{ker}(j)=i m(i)=D^{\prime}$ para todo $x \in \mathfrak{L}(E)$.

Mais ainda, temos que $k(\mathfrak{B}(E))=0$ pois $\mathfrak{B}(E)=\{x \in E$ tal que $x=d(y), y \in$ $E\}, \log 0 x \in \mathfrak{B}(E)$ implica $x=j k(y)$ e $k(x)=k j k(y)=(k j) k(y)=0$. 
Agora tome $x \in D^{\prime}$ e $y \in D$ tais que $i(y)=x$. Então $j(y) \in \mathfrak{L}(E)$ porque $d j(y)=$ $j k j(y)=0$, e a classe lateral de $j(y)(\bmod \mathfrak{B}(E))$ não depende da escolha de $y$ : basta ver que se $i(y)=i\left(y^{\prime}\right)=x$ então $j(y)(\bmod \mathfrak{B}(E))=j\left(y^{\prime}\right)(\bmod \mathfrak{B}(E))$ pois $i\left(y^{\prime}-y\right)=$ $x-x=0$ implica $y^{\prime}-y \in \operatorname{ker}(i)$, que implica $y^{\prime}-y \in \operatorname{Im}(k)$, portanto existe $z \in E$ tal que $k(z)=y^{\prime}-y$. Segue daí que $d(z)=j k(z)=j\left(y^{\prime}-y\right)=j\left(y^{\prime}\right)-j(y)$, de onde $j\left(y^{\prime}\right)-j(y) \in \mathfrak{B}(E)$ e daí se conclui que $j(y)(\bmod \mathfrak{B}(E))=j\left(y^{\prime}\right)(\bmod \mathfrak{B}(E))$. Denote esta classe por $j^{\prime}(x)$. Temos então um homomorfismo $j^{\prime}: D^{\prime} \rightarrow E^{\prime}$ dado por $x \mapsto j^{\prime}(x)$ que completa a construção de $\mathfrak{C}^{\prime}$.

Este processo pode ser aplicado a $\mathfrak{C}^{\prime}$ para obter um segundo par derivado $\mathfrak{C}^{\prime \prime}$ e assim por diante. Desta forma, temos uma sequência de pares exatos $\mathfrak{C}^{n}=<$ $D^{n}, E^{n}, i^{n}, j^{n}, k^{n}>, n=1,2, \ldots$ definida indutivamente por $\mathfrak{C}^{1}=\mathfrak{C}, \mathfrak{C}^{n}=\left(\mathfrak{C}^{n-1}\right)^{\prime}, n>$ 1.

Propriedades de $\left\{\mathfrak{C}^{n}\right\}$ :

1. $D=D^{1} \supset D^{2} \supset \ldots \supset D^{n} \supset D^{n+1} \supset \ldots$ onde $i^{n}: D^{n} \rightarrow D^{n}$ é a restrição de $i$ a $D^{n}$. Definimos $D^{\infty}=\bigcap_{i \in \mathbb{N}} D^{i}$.

2. $d^{n}=j^{n} k^{n}: E^{n} \rightarrow E^{n}$ é um operador diferencial em $E^{n}$ e mais ainda, $E^{n+1}$ é o grupo derivado de $E^{n}$ com respeito a $d^{n}$ e portanto temos uma sequência $E=E^{1}, E^{2}, \ldots, E^{n}, \ldots$ tal que $E^{n+1}=\mathfrak{H}\left(E^{n}\right)$ para todo $n>0$.

Definição 3.6. A seqüência $E=E^{1}, E^{2}, \ldots, E^{n}, \ldots$ tal que $E^{n+1}=\mathfrak{H}\left(E^{n}\right)$ para todo $n>0$ é aquela que definimos como a sequência espectral associada ao par exato $\mathfrak{C}$.

Segundo [12], em geral os grupos $E^{i}$ são denotados por $E_{r}^{p, q}$ onde $r$ denota o nível da seqüência espectral e $p$ e $q$ denotam a posição de $E^{i}$ dentro deste nível, formando assim para cada nível $r$ de uma seqüência espectral um diagrama como abaixo: 


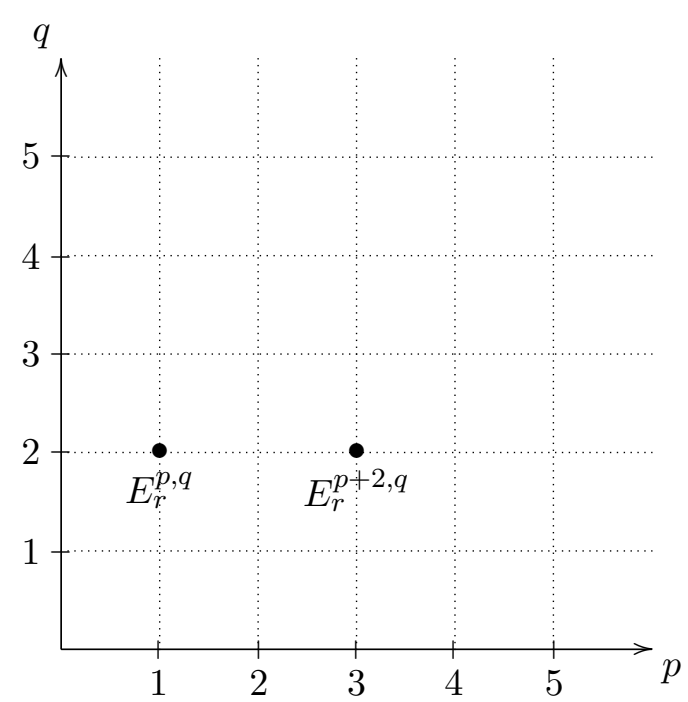

A diferencial $d_{r}: E_{r}^{*, *} \rightarrow E_{r}^{*, *}$ é em geral uma aplicação de bigrau $(r, r+1)$, ou seja, $d_{r}: E_{r}^{p, q} \rightarrow E_{r}^{p+r, q-r+1}$ e ainda, sempre que $r \geq 1$ temos que $E_{r+1}^{*, *}=$ $H\left(E_{r}^{*, *}, d_{r}\right)$, o que significa que

$$
E_{r+1}^{p, q}=\frac{k e r d_{r}: E_{r}^{p, q} \rightarrow E_{r}^{p+r, q-r+1}}{i m d_{r}: E_{r}^{p-r, q+r-1} \rightarrow E_{r}^{p, q}} .
$$

Quando $E_{r}^{p, q}$ é tal que $r>\max (p, q+1)$ então a diferencial $d_{r}$ se anula porque quando $q+1-r<0$ temos $E_{r}^{p+r, q-r+1}=\{0\}-$ com isto, $\operatorname{kerd}_{r}=E_{r}^{p, q}$. Ainda, como $p-r<0$, temos que $E_{r}^{p-r, q+r-1}=\{0\}$ e $i m d_{r}=\{0\}$, o que implica $E_{r+1}^{p, q}=E_{r}^{p, q}$ e portanto $E_{r+k}^{p, q}=E_{r}^{p, q}$ sempre que $k \geq 0$. Denotamos este nível especial por $E_{\infty}^{p, q}$

Definição 3.7. Uma seqüência espectral $\left\{E_{r}^{*, *}, d_{r}\right\}$ converge para um determinado nivel $E_{k}^{p, q}$ se a partir do nivel $k$ a diferencial $d_{r}$ é nula. 
Estudaremos a partir daqui uma seqüência espectral específica construída pela primeira vez por L. L. Larmore, razão pela qual a trataremos por seqüência de Larmore. Tal seqüência será a ferramenta que permitirá saber o número máximo de fibrados em cada caso, sendo portanto imprescindível para nosso intuito. A construção será feita a partir da criação de um par exato específico, e a existência da seqüência é então garantida como visto anteriormente.

A partir daqui, todos os espaços a serem considerados serão CW-complexos conexos com ponto base, todas as aplicações e todas as homotopias preservam os pontos base. Este capítulo é um detalhamento do que foi feito por L. L. Larmore em [11].

Definição 3.8. Considere uma torre de Postnikov para um espaço topológico $Y$. Para todo $n \geq 1$, denotamos por $\mathbf{G}_{\mathbf{n}}(\mathbf{Y})$ o sheaf sobre $Y_{1}$ cujo stalk sobre $y \in Y_{1}$ é $\pi_{n}\left(q^{-1}(y)\right)$, onde $q=q_{2} q_{3} \ldots q_{n-1} q_{n}: Y_{n} \rightarrow Y_{1}$, e

$$
\pi_{n}\left(q^{-1}(y)\right)=\left\{\begin{array}{cc}
\pi_{n}(Y) & \text { se } n \geq 2 \\
\pi_{1}\left(Y_{1}, y\right) & \text { se } n=1
\end{array}\right.
$$

Note que para o caso $n \geq 2$ acima, como $q=q_{2} q_{3} \ldots q_{n-1} q_{n}$ é uma fibração, o resultado $\pi_{n}\left(q^{-1}(y)\right)=\pi_{n}(Y)$ pode ser obtido de maneira completamente análoga àquela feita na proposição 24 para provar que a fibra de cada fibração $q_{n}$ é um espaço de Eilenberg-MacLane do tipo $\left(\pi_{n}(Y), n\right)$. Para o caso $n=1$, como a aplicação $q$ não faz sentido, definimos $\pi_{1}\left(q^{-1}(y)\right)=\pi_{1}\left(Y_{1}, y\right)$.

Agora fixaremos algumas notações, auxiliados pela figura 3.1.

Se temos $f: X \rightarrow Y_{1}, g: X \rightarrow Y$ ou $h: X \rightarrow Y_{m}(m \geq n)$ então denotamos respectivamente por $\pi_{\mathbf{n}}(\mathbf{Y}, \mathbf{f}), \quad \pi_{\mathbf{n}}(\mathbf{Y}, \mathbf{g})$ e $\pi_{\mathbf{n}}(\mathbf{Y}, \mathbf{h})$ o pullback $f^{-1}\left(G_{n}(Y)\right), \quad\left(P_{1} g\right)^{-1}\left(G_{n}(Y)\right)$ ou $\left(q_{2} \ldots q_{m} h\right)^{-1}\left(G_{n}(Y)\right)$.

Podemos olhar para a construção acima da seguinte maneira: o sheaf $G_{n}(Y)$ pode ser pensado como um fibrado de grupos com base $Y_{1}$, fibra $\pi_{n}(Y)$ e grupo estrutural $A u t\left(\pi_{n}(Y)\right)$, onde a ação do grupo estrutural é definida por uma representação do grupo fundamental da base, a saber: $\rho: \pi_{1}\left(Y_{1}\right) \rightarrow \operatorname{Aut}\left(\pi_{n}(Y)\right)$. Agora, como $Y_{1}=K\left(\pi_{1}(Y), 1\right)$ por definição, tais representações são na verdade as ações possíveis do grupo fundamental $\pi_{1}(Y)$ nos grupos de homotopia mais alta $\pi_{n}(Y)$, via a composição $p_{2} \ldots p_{m}$. A partir daí temos então o sheaf acima citado. Agora, suponha que $Y_{1}=B \Lambda$ para algum grupo finito $\Lambda$. Então todos os pullback sheaves $\pi_{n}(Y, f)$, como fibrados de grupos sobre $X$ são classificados por (classes de homotpia de) aplicações em $[X, B \Lambda]$, e como $B \Lambda=K(\Lambda, 1)$, temos que este último conjunto está em bijeção como conjunto $\operatorname{Hom}\left(\pi_{1}(X), \pi_{1}(B \Lambda)\right)=\operatorname{Hom}\left(\pi_{1}(X), \Lambda\right)$ - note que $\Lambda$ pode ser não abeliano. 


\section{Figura 3.1:}

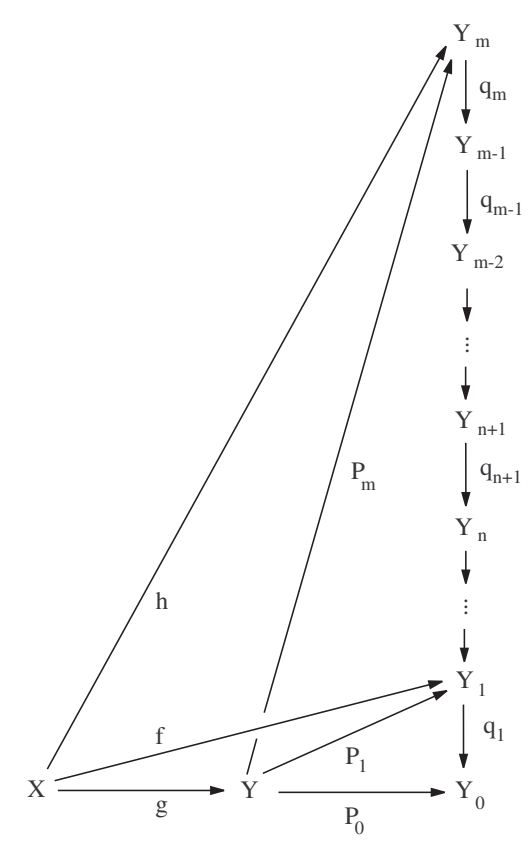

Sejam $f, g: X \rightarrow Y_{n}$ tais que $\left.f\right|_{A}=\left.g\right|_{A}$ para algum subcomplexo $A \in X$ e $n \geq 2$. Seja ainda $F: X \times I \rightarrow Y_{n-1}$ uma homotopia entre $q_{n} f$ e $q_{n} g$ que deixa $A$ fixo. Nestas condições, denotamos por $\delta^{\mathbf{n}}(\mathbf{f}, \mathbf{g} ; \mathbf{F}) \in H^{n}\left(X, A, \pi_{n}(Y, f)\right)$ a obstrução a um levantamento de $F$ a uma homotopia de $f \operatorname{com} g$ que deixa $A$ fixo. (Note então que a não existência de uma tal homotopia com relação à $F$ implica na existência de uma obstrução.)

Observação 3.3. Se $h: X \rightarrow Y_{n}$ é outra aplicação tal que $\left.h\right|_{A}=\left.f\right|_{A}$ e $G$ é uma homotopia de $q_{n} g$ com $q_{n} h$ que deixa A fixo, então $\delta^{n}(f, h ; F+G)=\delta^{n}(f, g ; F)+$ $\delta^{n}(g, h ; G)$, onde

$$
(F+G)(x, t)=\left\{\begin{array}{cl}
F(x, 2 t) & \text { se } 0 \geq t \geq 1 / 2 \\
G(x, 2 t-1) & \text { se } 1 / 2 \geq t \geq 1
\end{array}\right.
$$

Note que com isso temos $\delta^{n}(f, f+a ; C)+\delta^{n}(f+a, f ; C)=\delta^{n}(f, f ; C)=0$, de onde $\delta^{n}(f+a, f ; C)=-a$.

Definição 3.9. Seja $q: E \rightarrow B$ uma fibração. Duas aplicações $f_{0}, f_{1}: X \rightarrow E$ são ditas homotópicas por fibras $\left(f_{0} \sim_{q} f_{1}\right)$, se existe uma homotopia $F: f_{0} \sim f_{1}$ tal que 
$q F(x, t)=q F(x, 0)$ para todo $x \in X$ e $t \in I$ (portanto devemos ter necessariamente $\left.q f_{0}=q f_{1}\right)$.

Observação 3.4. Seja agora $f: X \rightarrow Y_{n}(n \geq 2)$ e a $\in H^{n}\left(X, A ; \pi_{n}(Y, f)\right)$. Denotamos por $\mathbf{f}+\mathbf{a}: X \rightarrow Y_{n}$ a aplicação (única a menos de homotopia de fibras com $A$ fixo) tal que $q_{n}(f+a)=q_{n} f$ e $\delta^{n}(f, f+a ; C)=a$, onde $C$ é a homotopia constante entre $q_{n} f$ e $q_{n} f$.

São válidas as seguintes propriedades:

- Se $b \in H^{n}\left(X, A ; \pi_{n}(Y, f)\right)$ então $f+(a+b)=(f+a)+b$ :

Para ver isto, observe que por definiçãof $+(a+b)$ é a única a menos de homotopia de fibras tal que $\delta^{n}(f, f+(a+b), C)=a+b$ e $q_{n}(f+(a+b))=q_{n} f$. Mas $q_{n}((f+a)+b)=q_{n}(f+a)=q_{n} f$, e além disso, $\delta^{n}(f,(f+a)+b, C)=$ $\delta^{n}(f, f+a, C)+\delta^{n}(f+a,(f+a)+b, C)=a+b$. Segue que devemos ter $f+(a+b)=(f+a)+b$.

- Se $\left(X^{\prime}, A^{\prime}\right)$ é outro par CW e $g:\left(X^{\prime}, A^{\prime}\right) \rightarrow(X, A)$ é uma aplicação, então $(f+a) g=f g+g^{*} a$. A situação aqui é descrita pela figura 3.2. Note que $q_{n}((f+a) g)=\left(q_{n}(f+a)\right) g=q_{n} f g=q_{n}\left(f g+g^{*} a\right)$. Mais ainda, devido às propriedades funtoriais de $g^{*}$ temos que $\delta^{n}(f g,(f+a) g ; C)=g^{*} a$, e portanto podemos concluir a igualdade $(f+a) g=f g+g^{*} a$, como indicado abaixo:

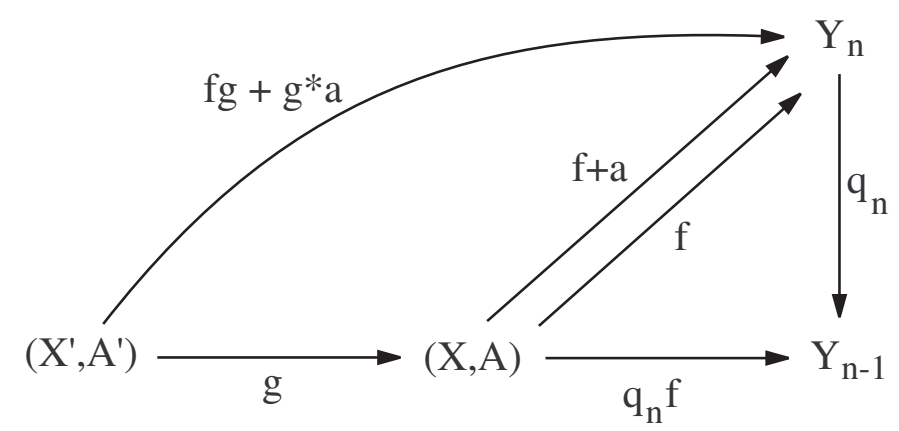

Figura 3.2: $(f+a) g=f g+g^{*} a$

Teorema 3.2. Para toda classe a $\in H^{n}\left(X, A ; \pi_{n}(Y, f)\right)$, $f+a$ é homotópica a $f($ rel $A)$ se e somente se $\delta^{n}(f, f ; F)=a$, onde $F$ é alguma homotopia de $q_{n} f$ com $q_{n} f$ que deixa A fixo. 
Note que o teorema nos diz que $f+a$ é homotópica a $f$ se e só se $a$ é obstrução a algum levantamento de homotopia entre $q_{n} f$ e $q_{n} f$ até $f$.

Demonstração. Seja $C$ a homotopia constante de $q_{n} f \operatorname{com} q_{n} f$, e $F$ alguma homotopia de $q_{n} f$ com $q_{n} f$ que deixa $A$ fixo.

$(\Rightarrow)$ Se $G$ é homotopia $(r e l A)$ de $f+a \operatorname{com} f$, então temos $\delta^{n}\left(f, f ; q_{n} G\right)=\delta^{n}(f, f+$ $a ; C)+\delta^{n}\left(f+a, f ; q_{n} G\right)=a+0=a$.

$(\Leftarrow)$ Basta observar que como $\delta^{n}(f+a, f ; F)=\delta^{n}(f+a, f ; C)+\delta^{n}(f, f ; F)=-a+a=$ 0 , então $F$ pode ser levantada a uma homotopia de $f+a \operatorname{com} f$.

Definição 3.10. Seja $\mathbf{L}_{f}$ o subgrupo de $H^{n}\left(X, A ; \pi_{n}(Y, f)\right)$ de todas as classes "a" tais que " $f+a$ " é homotópica a " $f(\operatorname{rel} A) "$.

Proposição 25. A função $(f+a) \mapsto\left(a+L_{f}\right)$ é uma bijeção entre o conjunto de todas as classes de homotopia (relA) de levantamentos de $q_{n} f$ a $Y_{n}$ que coincidem com $f$ em $A$ e o quociente

$$
\frac{H^{n}\left(X, A ; \pi_{n}(Y, f)\right)}{L_{f}}
$$

Demonstração. Seja $Z=\frac{H^{n}\left(X, A ; \pi_{n}(Y, f)\right)}{L_{f}} \mathrm{e}$

$$
W=\left\{[g] \text { tal que } g \text { é levantamento de } q_{n} f \text { a } Y_{n} \text { e } g|A=f| A\right\} .
$$

Seja $\varphi: Z \rightarrow W$ dada por $\varphi\left(a+L_{f}\right)=[f+a]$.

Para ver que $\varphi$ está bem definida, note que se $a+L_{f}=b+L_{f}$ então $a-b \in L_{f}$ e portanto temos $(f+a-b) \sim f$, ou seja, $f+a \sim f+b$, de onde $\varphi\left(a+L_{f}\right)=\varphi\left(b+L_{f}\right)$. (Note a existência de uma homotopia $f+a \sim f+b$ a partir daquela de $(f+a-b) \sim f$, como veremos no próximo parágrafo.)

Agora tome $a, b \in H^{n}\left(X, A ; \pi_{n}(Y, f)\right)$ tais que $a+L_{f} \neq b+L_{f}, \log a-b \notin L_{f}$ e daí $((f+a)-b) \not f$. Note que $\varphi\left(a+L_{f}\right)=[f+a]$ e $\varphi\left(b+L_{f}\right)=[f+b]$. Suponha por absurdo que $[f+a]=[f+b]$. Então existe homotopia $H: X \times$ $I \rightarrow Y_{n}$ tal que $H(x, 0)=f+a$ e $H(x, 1)=f+b$. Defina $H^{\prime}: X \times I \rightarrow Y_{n}$ por $H^{\prime}(x, t)=H(x, t)+(-b)$. Observe que $H^{\prime}$ é contínua pois $H$ é contínua e a cohomologia de $X \times I$ é a mesma de $X$ (porque $I$ é contrátil). Além disso $b \in$ $H^{n}\left(X, A, \pi_{n}(Y, f)\right)=H^{n}\left(X \times I, A, \pi_{n}(Y, f)\right)$ e portanto a soma $H^{\prime}=H+b$ é contínua. Com isto, $H^{\prime}(x, 0)=(f+a)-b$ e $H^{\prime}(x, 1)=f+(b-b)=f$, ou seja, $((f+a)-b) \sim f$, o que é absurdo. Segue que $\varphi$ é injetora.

Agora considere uma classe qualquer $[g] \in W$. Note que $g$ é então um levantamento de $q_{n} f$ a $Y_{n}$ tal que $g|A=f| A$. Como $q_{n} f=q_{n} g$ e $q_{n}$ é fibração, sabemos 
que podemos levantar a homotopia constante $C$ entre $q_{n} f$ e $q_{n} f$ a $Y_{n}$. Este levantamento pode ou não ser homotópico a $f(r e l A)$, e isso é decidido pela obstrução $a \in H^{n}\left(X, A ; \pi_{n}(Y, f)\right)$. Em todo caso, podemos escrever $g$ como $f+a$, onde $a$ é a obstrução descrita acima, com isto $[g] \sim[f+a]$. Mas então $\varphi\left(a+L_{f}\right)=[f+a]=[g]$. Com isto, temos que $\varphi$ é sobrejetora e injetora, portanto bijetora.

No caso em que $g: X \rightarrow Y$ (ou $h: X \rightarrow Y_{m}$ com $m \geq n$ ) é tal que $P_{n} g=f$ (ou $\left.q n+1 \ldots q_{m} h=f\right)$, usaremos a notação $L_{g}^{n}=L_{f}\left(\right.$ ou, respectivamente, $\left.L_{h}^{n}=L_{f}\right)$.

Corolário 2. Se $a \in H^{n}\left(X, A ; \pi_{n}(Y, f)\right)$, então $L_{f+a}=L_{f}$.

Demonstração. Sejam novamente $C$ a homotopia constante de $q_{n} f$ com $q_{n} f$, e $F$ qualquer homotopia de $q_{n} f \operatorname{com} q_{n} f$ que deixa $A$ fixo. Então segue que $\delta^{n}(f+a, f+$ $a ; F)=\delta^{n}(f+a, f ; C)+\delta^{n}(f, f ; F)+\delta^{n}(f, f+a ; C)=-a+\delta^{n}(f, f ; F)+a=$ $\delta^{n}(f, f ; F)$. Note que agora o teorema 3.2 nos permite concluir a igualdade entre $L_{f+a}$ e $L_{f}$.

\subsection{Construção da seqüência de Larmore}

Nesta seção, construiremos uma seqüência espectral munida das seguintes propriedades:

3.2.1. ${ }^{f} E_{2}^{p, q}=E_{2}^{p, q}=H^{p}\left(X, A, \pi_{q}(Y, f)\right)$, se $2 \leq q \leq n$, e ainda $1 \leq p \leq q+1$;

3.2.2. $E_{2}^{p, q}=0$ para qualquer outro valor de $p$ e $q$;

3.2.3. $d_{r}: E_{r}^{p, q} \rightarrow E_{r}^{p+r, q+r-1}$ sempre que $r \geq 2$.

3.2.4. $E_{\infty}^{n, n}=\frac{H^{n}\left(X, A ; \pi_{n}(Y, f)\right)}{L_{f}}$, que pelo teorema 3.2 e pela definição de $L_{f}$, está em bijeção com o conjunto das classes de homotopia (relA) de aplicações $X \rightarrow Y_{n}$ cuja projeção a $Y_{n-1}$ é homotópica $(\operatorname{rel} A)$ a $q_{n} f$.

Observação 3.5. Aproveitaremos esta bijeção proposta pela última propriedade para explicitar algumas notações e convenções que usaremos nesta seção.

Denotamos por $[\mathbf{X} ; \mathbf{Z}: \mathbf{g}]$ o conjunto das classes de homotopia (relA) de aplicações $f: X \rightarrow Z$ tais que $f \mid A=g: A \rightarrow Z$.

Considere a função $\left(q_{n}\right)_{\#}:\left[X ; Y_{n}:\left.f\right|_{A}\right] \rightarrow\left[X ; Y_{n-1}:\left.q_{n} f\right|_{A}\right]$ dada por $\left(q_{n}\right)_{\#}(g)=$ $\left[q_{n} g\right]$. Note que $\left(q_{n}\right)_{\#}$ está bem definida pois se $g_{1}, g_{2} \in[g]$ então $g_{1} \sim g_{2}($ relA $)$ e $\left.g_{1}\right|_{A}=\left.g_{2}\right|_{A}=\left.f\right|_{A}$. Mais ainda, $\left(q_{n}\right)_{\#}\left(g_{1}\right)=q_{n} g_{1}: X \rightarrow Y_{n-1} e\left(q_{n}\right)_{\#}\left(g_{2}\right)=q_{n} g_{2}:$ 
$\left.X \rightarrow Y_{n-1} \operatorname{com} q_{n} g_{1}\right|_{A}=\left.q_{n} g_{2}\right|_{A}=\left.q_{n} f\right|_{A}$. Agora, se $H$ é homotopia (relA) entre $g_{1}$ e $g_{2}$ então $q_{n} H$ é homotopia (relA) entre $q_{n} g_{1}$ e $q_{n} g_{2}$, logo $\left(q_{n}\right)_{\#}$ está bem definida.

Observe que $\left(q_{n}\right)_{\#}$ é só uma função entre conjuntos, mas $\left(q_{n}\right)_{\#}^{-1}\left(q_{n} f\right)$ é um grupo abeliano onde a classe de homotopia de $f$ é 0 . Isso acontece porque $\left(q_{n}\right)_{\#}^{-1}\left(q_{n} f\right)$ é o conjunto das classes de homotopia (relA) de levantamentos de $q_{n} f$ a $Y_{n}$ que mantém A fixo, e esse conjunto, como já vimos antes, pode ser identificado com o quociente $\frac{H^{n}\left(X, A ; \pi_{n}(Y, f)\right)}{L_{f}}$ (note que o zero neste quociente corresponde às aplicações que são homotópicas a $f$ ).

Agora podemos começar a construção da nossa seqüência espectral, que será obtida via um par exato como o da figura abaixo:

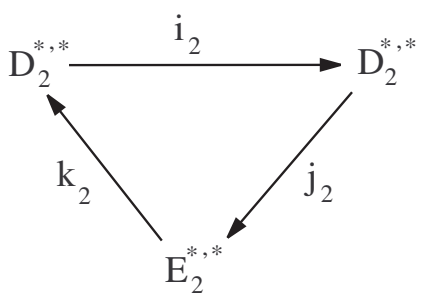

Para isto, podemos então definir $E_{2}^{p, q}$ pelas propriedades vistas em 3.2.1 e 3.2.2. Ou seja, temos que

$$
{ }^{f} E_{2}^{p, q}=E_{2}^{p, q}=\left\{\begin{array}{cl}
H^{p}\left(X, A, \pi_{q}(Y, f)\right) & \text { se } 2 \leq q \leq n \text { e ainda } 1 \leq p \leq q+1 \\
0 & \text { para qualquer outro valor de } p \text { e } q
\end{array}\right.
$$

Precisamos definir agora $D_{2}^{p, q}$ e as aplicações $i_{2}, j_{2}$ e $k_{2}$, sendo que para estas últimas devemos ter que $\operatorname{bideg}\left(i_{2}\right)=(-1,-1), \quad \operatorname{bideg}\left(j_{2}\right)=(2,1) \quad$ e $\operatorname{bideg}\left(k_{2}\right)=$ $(0,0)$.

Considere para todo $t \leq n$, o conjunto $M_{t}=m_{*}\left(X, Y_{t}\right)$ de todas as aplicações $h: X \rightarrow Y_{t}$ tais que $\left.h\right|_{A}=\left.q_{t}^{n} f\right|_{A}$, com a topologia compacto-aberto (note que $q_{t}^{n}$ é a notação para $\left.q_{t+1} q_{t+2} \ldots q_{n-1} q_{n}: Y_{n} \rightarrow Y_{t}\right)$.

Definimos agora

3.2.5. $D_{2}^{p, q}=\pi_{q-p}\left(M_{q}, q_{q}^{n} f\right)$ se $0 \leq q \leq n$ e $p \leq q$.

3.2.6. $D_{2}^{p, q}=0$ se $q<p$ ou $q<0$.

3.2.7. $D_{2}^{p, q}=D_{2}^{p-1, q-1}$ se $q>n$. 
Observe que em $\pi_{q-p}\left(M_{q}, q_{q}^{n} f\right), q_{q}^{n} f$ é apenas o ponto base do $(q-p)$-ésimo grupo de homotopia. Será comum daqui por diante identificarmos $\pi_{q-p}\left(M_{q}, q_{q}^{n} f\right)$ com o conjunto das classes de homotopia de aplicações do tipo $g: X \times I^{q-p} \rightarrow Y_{q}$ - essa identificação é uma aplicação direta do funtor adjunto. Mais ainda, por vezes também olharemos para os elementos deste conjunto como sendo as classes do tipo $\left[X, Y_{q} ; q_{q}^{n} f\right]$, já descritas anteriormente.

Uma última observação a ser feita neste ponto é que em $3.2 .5, D_{2}^{p, q}$ é um grupo se $q=p+1$ e é apenas um conjunto se $q=p$, mas este fato não altera em nada a construção da nossa seqüência espectral.

Agora passaremos a definir as aplicações $i_{2}, j_{2}$ e $k_{2}$ :

3.2 .8 .

$$
i_{2}=\left\{\begin{array}{cc}
I d: D_{2}^{p, q} \rightarrow D_{2}^{p-1, q-1} & \text { se } q>n \\
\left(q_{q}\right)_{\#}:\left[X, Y_{q} ; q_{q}^{n} f\right] \rightarrow\left[X, Y_{q-1} ; q_{q-1}^{n} f\right] & \text { se } q \leq n
\end{array}\right.
$$

Veja que $i_{2}$ está bem definida no segundo caso, pois já mostramos a boa definição de $\left(q_{q}\right)_{\#}$. Para ver que o bigrau de $i_{2}$ é $(-1,-1)$, precisamos avaliar dois casos:

- Caso 1: Quando $q>n$ temos que $i_{2}=i d$ mas também temos que $D_{2}^{p, q}=$ $D_{2}^{p-1, q-1}$, e portanto $\operatorname{bideg}\left(i_{2}\right)=(-1,-1)$.

- Caso 2: Quando $q \leq n$, temos $i_{2}=\left(q_{q}\right)_{\#}:\left[X ; Y_{q}: q_{q}^{n} f\right] \rightarrow\left[X ; Y_{q-1}: q_{q-1}^{n} f\right]$. Observe que estamos identificando $\pi_{q-p}\left(M_{q}, q_{q}^{n} f\right) \operatorname{com}\left[X, Y_{q}, q_{q}^{n} f\right]$.

Note que utilizando as identificações anteriores, temos

$$
\begin{gathered}
i_{2}=\left(q_{q}\right)_{\#}:\left[X, Y_{q} ; q_{q}^{n} f\right]=\pi_{q-p}\left(M_{q}, q_{q}^{n} f\right)=D_{2}^{p, q} \longrightarrow\left[X, Y_{q-1} ; q_{q-1}^{n} f\right]= \\
=\pi_{q-p}\left(M_{q-1}, q_{q-1}^{n} f\right)=D_{2}^{p-1, q-1} .
\end{gathered}
$$

Note que o índice $(p-1, q-1)$ de $D_{2}^{*, *}$ nesta última igualdade se deve ao fato de que sabemos que o índice do grupo de homotopia é $p-q$ (já que $D_{2}^{*, *}$ é definido como o $(p-q)$-ésimo grupo de homotopia), assim como sabemos que o índice do espaço $M_{t}$ neste caso é $t=q-1$, então devemos ter necessariamente um índice $p-1$ para termos o índice do grupo de homotopia igual a $(q-1)-(p-1)=q-p$.

Fica claro a partir daí que o bigrau de $i_{2}$ é $(-1,-1)$.

Considere agora:

1. O isomorfismo

$S^{q-p}: H^{p+2}\left(X, A, \pi_{q+1}(Y, f)\right) \rightarrow H^{q+2}\left(X \times I^{q-p}, X \times \partial I^{q-p} \bigcup A \times I^{q-p} ; \pi_{q+1}(Y, g)\right)$, 
que é a suspensão de $(q-p)$-folhas (é muito importante perceber que este isomorfismo relaciona a obstrução a um levantamento de homotopia entre $q_{n} f$ e $q_{n} f$ a uma obstrução a um levantamento de homotopia entre $q_{n} g$ e $q_{n} g$ ) e,

2. Se $g: X \times I^{q-p} \rightarrow Y_{q}$ é tal que $g(z, s)=q_{q}^{n} f(z)$ para todo $(z, s) \in\left(X \times \partial\left(I^{q-p}\right)\right) \bigcup\left(A \times\left(I^{q-p}\right)\right)$, considere $\gamma^{q+2}(g)$ como sendo a obstrução a encontrar um levantamento $h: X \times I^{q-p} \rightarrow Y_{q+1}$ de $g$ tal que $h(x, v)=q_{q+1}^{n} f(x)$ para todo $(x, v) \in\left(X \times \partial\left(I^{q-p}\right)\right) \bigcup\left(A \times\left(I^{q-p}\right)\right)$.

Com isto podemos definir:

\subsection{9.}

$$
j_{2}: D_{2}^{p, q}=\pi_{q-p}\left(M_{q}, q_{q}^{n} f\right) \longrightarrow E_{2}^{p+2, q+1}=H^{p+2}\left(X, A ; \pi_{q+1}(Y, f)\right)
$$

por $j_{2}(x)=\left(S^{q-p}\right)^{-1} \gamma^{q+2}(g)$, onde se $p \leq q$ e $0 \leq q<n$ (veja o caso 3.2.5) então pelo que vimos acima, um ponto $x \in D_{2}^{p, q}$ é representado por uma aplicação $g$ : $X \times I^{q-p} \rightarrow Y_{q}$ tal que $g(z, s)=q_{q}^{n} f(z)$ para todo $(z, s) \in\left(X \times \partial\left(I^{q-p}\right)\right) \bigcup\left(A \times\left(I^{q-p}\right)\right)$.

Note que $j_{2}$ está bem definida porque tanto $\gamma$ como $S^{q-p}$ estão bem definidas. Mais ainda, temos que se $p>q$ ou se $q<0$ ou se $q \geq n$ então $j_{2}$ é necessariamente a aplicação nula, já que $E_{2}^{p+2, q+1}=0$. Segue da definicão de $j_{2}$ que seu bigrau é $(2,1)$.

É claro que a obstrução $\gamma^{q+2}(g)$ é zero se e só se $g$ pode ser levantada, como isto, temos que a seqüência $D_{2}^{p+1, q+1} \stackrel{i_{2}}{\longrightarrow} D_{2}^{p, q} \stackrel{j_{2}}{\longrightarrow} E_{2}^{p+2, q+1}$ é exata, ou seja, $\operatorname{Im}\left(i_{2}\right)=$ $\operatorname{Ker}\left(j_{2}\right)$ :

$\left(\operatorname{Im}\left(i_{2}\right) \subset \operatorname{Ker}\left(j_{2}\right)\right):$ Este resultado é trivial se $E_{2}^{p+2, q+1}$ é nulo. No único caso em que $E_{2}^{p+2, q+1}$ não é nulo, temos que qualquer $y \in \operatorname{Im}\left(i_{2}\right)=\operatorname{Im}\left(\left(q_{q}\right)_{\#}\right)$ pode ser levantado (pela definição de $\left.\left(q_{q}\right)_{\#}\right)$ logo não existe obstrução e portanto $j_{2}(y)=0$.

$\left(\operatorname{Ker}\left(j_{2}\right) \subset \operatorname{Im}\left(i_{2}\right)\right)$ Nos casos em que $j_{2}$ é nula, temos que $i_{2}$ é a identidade e portanto vale o resultado. Nos casos em que $j_{2}$ não é nula, então $y \in \operatorname{Ker}\left(j_{2}\right)$ pode ser levantado já que $j_{2}(y)=\left(S^{q-p^{-1}} \gamma^{q+2}\right)(y)=0$ e como $S^{q-p}$ é isomorfismo, temos que $\gamma^{q+2}(y)=0$. Se $y$ pode ser levantado a um certo $x_{y}$ segue que $i_{2}\left(x_{y}\right)=\left(q_{q}\right)_{\#}\left(x_{y}\right)=y$.

Observação 3.6. Como toda homotopia ( $\mathrm{rel} A$ ) de $q_{n} f$ com $q_{n} f$ representa um laço em $M_{n-1}$, então temos que $L_{f}$ é a imagem de $j_{2}: D_{2}^{n-2, n-1}=\pi_{1}\left(M_{n-1}, q_{n} f\right) \longrightarrow$ $E_{2}^{n, n}=H^{n}\left(X, A, \pi_{n}(Y, f)\right)$ :

( $):$ Seja $a \in \operatorname{Im}\left(j_{2}\right)$. Portanto $a=j_{2}(b)=S^{1}\left(\gamma^{n}(b)\right)$, onde $b \in \pi_{1}\left(M_{n-1}, q_{n} f\right)$ é uma homotopia entre $q_{n} f$ e $q_{n} f$ tal que $f+a=f+j_{2}(b)=f+S^{1}\left(\gamma^{n}(b)\right.$ ) (note que a obstrução a levantar a homotopia é a mesma obstrução a levantar a aplicação 
$b: X \times I \rightarrow Y_{n-1}$, o que está ocorrendo é apenas uma identificação.). Com isto, devemos ter que $\delta^{n}(f, f ; b)=$ a e portanto $a \in L_{f}$.

$(\subset):$ Seja $a \in L_{f}$. Então temos que $a=\delta^{n}(f, f ; F)$ para alguma $F: X \times I \rightarrow$ $Y_{n-1} \operatorname{com} f(x, 0)=F(x, 1)=q_{n} f$. Dai temos que $a=j_{2}(F)=S^{1}\left(\delta^{n}(F)\right)$.

Para todo $2 \leq q \leq n, 1 \leq p \leq q$ e qualquer $a \in E_{2}^{p, q}=H^{p}\left(X, A, \pi_{q}(Y, f)\right)$ seja

$$
b=S^{q-p}(a) \in H^{q}\left(X \times I^{q-p},\left(X \times \partial I^{q-p}\right) \bigcup\left(A \times I^{q-p}\right) ; \pi_{q}(Y, C)\right),
$$

onde $C(x, v)=q_{q}^{n} f(x)$ para todo $(x, v) \in X \times I^{q-p}$.

3.2.10. Defina

$$
k_{2}: E_{2}^{p, q}=H^{p}\left(X, A ; \pi_{q}(Y, f)\right) \longrightarrow D_{2}^{p, q}=\pi_{q-p}\left(M_{q}, q_{q}^{n} f\right)
$$

por

$$
k_{2}(a)=[C+b]
$$

Note que podemos definir $k_{2}$ desta maneira pois $C: X \times I^{q-p} \rightarrow Y_{q}$ e $b \in H^{q}\left(X \times I^{q-p},\left(X \times \partial I^{q-p}\right) \bigcup\left(A \times I^{q-p}\right) ; \pi_{q}(Y, C)\right)$. Além disso, $k_{2}$ está bem definida porque se trata da composição de duas aplicações bem definidas, a saber, da suspensão $S^{q-p}$ e da aplicação $a \mapsto[C+a]$.

Se $p=q$ então $E_{2}^{p, q}$ é apenas um conjunto e, finalizando, se $p>q$ temos que $k_{2}$ é a aplicação nula. Nosso próximo intuito é mostrar que $\operatorname{Im}\left(k_{2}\right)=\operatorname{Ker}\left(i_{2}\right)$. Para isto, veja que como $q_{q}(C+b)=q_{q} C$, segue imediatamente que

\subsubsection{1. $\operatorname{Im}\left(k_{2}\right) \subset \operatorname{Ker}\left(i_{2}\right)$}

Agora mostraremos que $\operatorname{ker}\left(i_{2}\right) \subset \operatorname{Im}\left(k_{2}\right)$ e portanto vale a igualdade $\operatorname{Im}\left(k_{2}\right)=$ $\operatorname{Ker}\left(i_{2}\right)$. Seja $[a] \in \operatorname{Ker}\left(i_{2}\right)$. Então um elemento $g$ que representa $[a]$ é da forma $g: X \times I^{q-p} \rightarrow Y_{q}$. Note que $q_{q}(g)$ é homotópico $\left(\operatorname{rel}\left(X \times \partial I^{q-p}\right) \cup\left(A \times I^{q-p}\right)\right)$ a $q_{q} C$ via uma homotopia $F$ (isso porque $[a] \in \operatorname{Ker}\left(i_{2}\right)$. Observe ainda o abuso de notação decorrente da identificação de conjuntos.).

Temos então que $\delta^{q}(C, g, F) \in H^{q}\left(X \times I^{q-p},\left(X \times \partial I^{q-p}\right) \bigcup\left(A \times I^{q-p}\right) ; \pi_{q}(Y, C)\right)$ e portanto $\left(S^{q-p}\right) \delta^{q}(C, g, F) \in H^{p}\left(X, A, \pi_{q}(Y, f)\right)=E_{2}^{p, q}$. Agora veja que $k_{2}\left(S^{q-p}\right) \delta^{q}(C, g, F)$ é o elemento de $D_{2}^{p, q}$ representado pela aplicação $C+\delta^{q}(C, g, F)$ e esta aplicação é nada menos que a própria $g$, portanto segue que $[a]=k_{2}\left(S^{q-p}\right) \delta^{q}(C, g, F)$.

A partir daqui nosso objetivo é mostrar que

3.2.12. $\operatorname{Ker}\left(k_{2}\right)=\operatorname{Im}\left(j_{2}\right)$ se $p \leq q$. 
Sejam então $2 \leq q \leq n, \quad 1 \leq p \leq q$ e $g(x, v)=q_{q}^{n} f(x) \in Y_{q}$ para todo $(x, v) \in X \times I^{q-p}$; observe que $g$ representa o zero em $D_{2}^{p, q}=\pi_{q-p}\left(M_{q}, q_{q}^{n} f\right)$.

Para mostrar que a igualdade 3.2.12 é válida, seguiremos a seguinte linha de raciocínio: primeiro mostraremos que $b \in \operatorname{Ker}\left(k_{2}\right) \Longleftrightarrow S^{p-q}(b) \in L_{g}$, e depois mostraremos que $S^{p-q}(b) \in L_{g} \Longleftrightarrow b \in \operatorname{Im}\left(j_{2}\right)$.

$b \in \operatorname{Ker}\left(k_{2}\right) \Longleftrightarrow S^{p-q}(b) \in L_{g}$ :

Veja que $C(x, v)=g(x, v): X \times I^{q-p} \rightarrow Y_{q}$ leva $(x, v)$ em $q_{q}^{n} f(x)$. Com isto, se $b \in \operatorname{ker}\left(K_{2}\right)$ então $\left[C+\gamma^{q+2}(b)\right]=\left[q_{q}^{n} f\right]=[C]$, de onde $\gamma^{q+2}(b) \in L_{C}=L_{g}$. Por outro lado, se $S^{q-p}(b) \in L_{g}$, então $g+S^{q-p}(b)$ é homotópica a $g=C=[0]$, de onde $b \in \operatorname{ker}\left(k_{2}\right)$

$$
S^{p-q}(b) \in L_{g} \Longleftrightarrow b \in \operatorname{Im}\left(j_{2}\right):
$$

Se $b=j_{2}(a) \in \operatorname{Im}\left(j_{2}\right)$ então $a$ é a classe de uma homotopia $F\left(\operatorname{rel} X \times I^{q-p} \cup A \times\right.$ $\left.I^{q-p}\right)$ de $q_{q} g$ com $q_{q} g$. Agora daí veja que $S^{q-p}(b)=\delta^{q}(g, g ; F) \in L_{g}$ - isso porque $S^{q-p}(b)=S^{q-p}\left(\left(S^{q-p}\right)^{-1} \gamma^{q+2}(F)\right)=\gamma^{q+2}(F)$ que corresponde a $\delta^{q+2}(g, g, F) \in L_{g}$.

Por outro lado, se $S^{q-p}(b) \in L_{g}$ então para alguma homotopia $F\left(\right.$ rel $X \times \partial I^{q-p} \cup$ $\left.A \times I^{q-p}\right)$ de $q_{q} g$ com $q_{q} g$ temos $S^{q-p}(b)=\partial^{q}(g, g ; F)$. Agora basta considerar um representante " $a$ " de $[F] \in D^{p-2, q-1}$ e daí temos $j_{2}(a)=b$.

Agora que já temos a exatidão provada, basta notar que pela definição de ${ }^{f} E_{2}^{p, q}$, o mesmo se anula para valores de $p$ e $q$ que não satisfazem $1 \leq p \leq q+1$ com $2 \leq q \leq n$. Portanto apenas finitos elementos $E_{2}$ serão não-nulos. Assim, depois de um número finito de passos, obtemos $E_{\infty}$. Também obtemos neste ponto uma seqüência exata

$$
0 \rightarrow E_{\infty} \stackrel{k_{\infty}}{\longrightarrow} D \infty \stackrel{i_{\infty}}{\longrightarrow} D_{\infty} \rightarrow 0
$$

Consideremos agora o seguinte diagrama com colunas exatas:

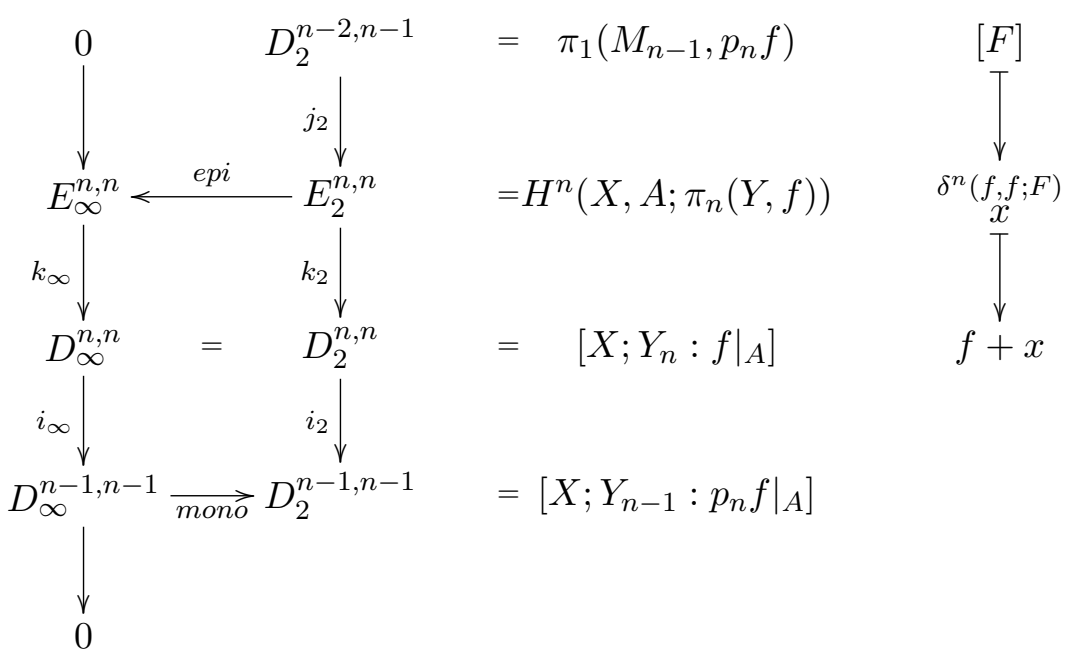


Um elemento típico de $D_{2}^{n-2, n-1}$ é uma classe de homotopia rel $((X \times \partial I) \cup(A \times$ $I))$ de homotopias de $p_{n} f$ com ele mesmo. Se $F$ é uma homotopia assim, pela definição de $j_{2}$ temos $j_{2}[F]=\delta^{n}(f, f ; F)$. Agora se $x \in H^{n}\left(X, A ; \pi_{n}(Y, f)\right)$, então $k_{2}(x)=f+x$ pela definição de $k_{2}$. Com isso temos que vale $\operatorname{Im}\left(j_{2}\right)=L_{f}$, e ainda $E_{\infty}^{n, n}=\frac{H^{n}\left(X, A ; \pi_{n}(Y, f)\right)}{L_{f}}$, ou seja, o conjunto das classes de homotopia $(r e l A)$ de levantamentos de $p_{n} f$.

Observações finais sobre a construção da seqüência espectral:

- Se $g:\left(X^{\prime}, A^{\prime}\right) \rightarrow(X, A)$ é uma aplicação, então $g$ induz uma aplicação de seqüências espectrais:

- $g^{*}:{ }^{f} E_{r}^{p, q} \rightarrow{ }^{f g} E_{r}^{p, q}$ para todo $p, q, r$. Se ainda temos $h: Y \rightarrow Z$ (onde $Z$ é qualquer outro espaço), então $h$ determina uma aplicação $h_{m}:(Y)_{m} \rightarrow$ $(Z)_{m}$ para todo $m \geq 0$. A partir daí $h_{\#}: \pi_{1}\left(Y, y_{0}\right) \rightarrow \pi_{1}\left(Z, z_{0}\right)$ induz um homomorfismo de sheaves de $G_{n}(Y)$ para $\left(h_{1}\right)^{-1} G_{n}(Z)$ que por sua vez induz o homomorfismo:

- $h_{*}: H^{*}\left(X, A, \pi_{m}(Y, f)\right) \rightarrow H^{*}\left(X, A, \pi_{m}(A, h f)\right)$ para todo $m \geq 0$ e ainda uma aplicação entre seqüências espectrais:

- $h_{*}:{ }^{f} E_{r}^{p, q} \rightarrow{ }^{h f} E_{r}^{p, q}$ para todo $p, q, r$.

A partir das técnicas até aqui apresentadas, podemos calcular todas as classes de homotopia que preservam ponto base de um espaço de dimensão finita $X$ até um espaço $Y$. A seguir vamos esclarecer como conseguir as classes de homotopia livres, como feito em [11].

Seja $f: X \rightarrow Y$ qualquer aplicação que preserva ponto base e seja $a \in \pi_{1}\left(Y, y_{0}\right)$. Pela propriedade de extensão da homotopia, podemos encontrar uma homotopia livre $F: X \times I \rightarrow Y$ de $f$ tal que $\left.F\right|_{\left\{x_{0}\right\} \times I}$ representa $a$. Seja $f^{a}(x)=F(x, 1)$ para qualquer $x \in X$; $f^{a}$ é única a menos de homotopia que preserva ponto base, e $f^{a b} \sim\left(f^{a}\right)^{b}$ para qualquer outro $b \in \pi_{1}\left(Y, y_{0}\right)$.

O teorema a seguir foi retirado de [11]:

Teorema 3.3. Se $f$ e $g$ são quaisquer aplicações que preservam ponto base de $X$ para $Y$, então $f$ é livremente homotópica a $g$ se e só se $f^{a}$ é homotópica por ponto base a $g$ para algum $a \in \pi_{1}\left(Y, y_{0}\right)$.

Demonstração. Se $f^{a}$ é homotópica por ponto base a $g$, então $f$ é obviamente livremente homotópica a $g$ uma vez que $f$ é livremente homotópica a $f^{a}$. Por outro lado, se $F: X \times I \rightarrow Y$ é uma homotopia livre de $f$ com $g$, seja $a$ aquele elemento de $\pi_{1}\left(Y, y_{0}\right)$ representado pelo laço $\left.F\right|_{\left\{x_{0}\right\} \times I}$. Então $f^{a}=g$ a menos de homotopia que preserva ponto base. 
O seguinte resultado segue naturalmente da teoria de obstrução:

Teorema 3.4. Se $n \geq 2, f: X \rightarrow Y_{n}$ é uma aplicação, $a \in H^{n}\left(X, x_{0} ; \pi_{n}(Y, f)\right)$ e ainda $b \in \pi_{1}\left(Y, y_{0}\right)$, então $(f+a)^{b}=f^{b}+1_{*}^{b}(a)$, onde $1_{*}^{b}$ é o homomorfismo induzido pela aplicação $1^{b}$ e 1 é a identidade em $Y_{n}$.

\subsection{Exemplos}

Agora exibiremos os exemplos trabalhados por nós logo após o estudo da construção da seqüência espectral de Larmore. Estes foram os primeiros resultados obtidos por nós e constituíram um teste das ferramentas apresentadas até aqui.

Seja $Q=\{ \pm 1, \pm i, \pm j, \pm k\}$ o grupo dos quatérnios, $\frac{S^{3}}{Q}=O$ o espaço quaterniônico e seja ainda $G$ um dos elementos do conjunto $J=\left\{S^{3}, U_{2}, S O_{3}\right\}$. Nosso interesse é descrever o conjunto $[O, B G]$ das classes de homotopia de aplicações de $O$ em $B G$, sendo este último o espaço de classificação de $G$. Como $\pi_{1}(B G)=0$, então $[O, B G]=[O, B G]_{0}$ e mais ainda, não precisaremos usar coeficientes locais para nenhum dos possíveis grupos $G$. Observe que para o caso $G=O_{3}$, o grupo fundamental não é trivial e portanto há necessidade de se usar coeficientes locais.

Nosso primeiro passo é construir uma torre de Postnikov para $B G$, e para isso levamos em consideração que $\pi_{1}(B G)=0$ e que a fibra de qualquer $p_{n}$ é um espaço de Eilemberg-McLane do tipo $K\left(\pi_{n}(B G), n\right)$. Com isto, temos a seguinte torre:

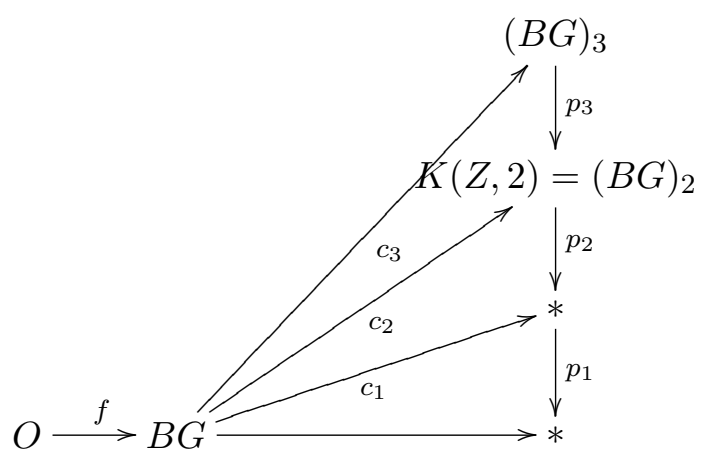

Onde

$$
Z=\pi_{2}(B G)=\pi_{1}(G)= \begin{cases}0 & \text { se } G=S^{3} \\ \mathbb{Z} & \text { se } G=U_{2} \\ \mathbb{Z}_{2} & \text { se } G=S O_{3}\end{cases}
$$


Sabemos que $\left[O,(B G)_{2}\right]=[O, K(Z, 2)]_{0}=H^{2}(O, Z)$ e portanto basta calcularmos este último grupo para cada elemento do conjunto $J$. Para isto, note primeiramente que

$$
H_{n}(O ; \mathbb{Z})= \begin{cases}\mathbb{Z}, & \text { se } n=0 \\ \mathbb{Z}_{2} \oplus \mathbb{Z}_{2}, & \text { se } n=1 \\ 0, & \text { se } n=2 \\ \mathbb{Z}, & \text { se } n=3\end{cases}
$$

Como $O$ é uma variedade fechada que mergulha em $R^{4}$ com codimensão 1 , então $O$ é orientável e portanto podemos aplicar a dualidade de Poincaré $H_{q}(O) \approx$ $H^{3-q}(O)$, o que nos leva até:

$$
H^{n}(O ; \mathbb{Z})= \begin{cases}\mathbb{Z}, & \text { se } n=0 \\ 0, & \text { se } n=1 \\ \mathbb{Z}_{2} \oplus \mathbb{Z}_{2}, & \text { se } n=2 \\ \mathbb{Z}, & \text { se } n=3\end{cases}
$$

Disso segue imediatamente que $\left[O,(B G)_{2}\right]=[O, K(Z, 2)]=H^{2}(O, Z)=\mathbb{Z}_{2} \oplus \mathbb{Z}_{2}$ quando $G=U_{2}$. É claro que se $G=S^{3}$ então $Z=\pi_{1}\left(S^{3}\right)=0$ de onde $\left[O,(B G)_{2}\right]=$ 0.

Agora, pelo teorema dos coeficientes universais, temos que

$$
H^{2}\left(O, \mathbb{Z}_{2}\right) \approx\left(H^{2}(O) \otimes \mathbb{Z}_{2}\right) \oplus\left(\operatorname{Tor}\left(H^{3}(O) ; \mathbb{Z}_{2}\right)\right)
$$

onde é claro que $\operatorname{Tor}\left(H^{3}(O) ; \mathbb{Z}_{2}\right)=\operatorname{Tor}\left(\mathbb{Z} ; \mathbb{Z}_{2}\right)=0$ e que $H^{2}(O) \otimes \mathbb{Z}_{2}=\left(\mathbb{Z}_{2} \oplus \mathbb{Z}_{2}\right) \otimes$ $\mathbb{Z}_{2}=\mathbb{Z}_{2} \oplus \mathbb{Z}_{2}$. Concluímos daí que se $G=S O_{3}$ então $[O, K(Z, 2)]_{0}=\mathbb{Z}_{2} \oplus \mathbb{Z}_{2}$.

Temos então até agora:

$$
\left[O,(B G)_{2}\right]= \begin{cases}0, & \text { se } G=S^{3} \\ \mathbb{Z}_{2} \oplus \mathbb{Z}_{2}, & \text { se } G=U_{2} \\ \mathbb{Z}_{2} \oplus \mathbb{Z}_{2}, & \text { se } G=S O_{3}\end{cases}
$$

Este mesmo resultado poderia ter sido obtido utilizando a seqüência espectral de Larmore. Mostraremos isso para o caso $G=U_{2}$ : a sequência será construída para 
$\mathrm{n}=2$ (onde o índice é o mesmo de $(B G)_{2}$ ), e a aplicação $f$ será a trivial $c_{0}$, de onde $\pi_{q}\left(B U_{2}, c_{0}\right)=\pi_{q}\left(B U_{2}\right)$. Temos que

$$
\begin{cases}E_{2}^{p, q}=H^{p}\left(O, \pi_{q}\left(B U_{2}\right)\right), & \text { se } 2 \leq q \leq 2 \text { ou seja, } q=2 \text { e ainda } 1 \leq p \leq 3 \\ E_{2}^{p, q}=0, & \text { caso contrário }\end{cases}
$$

e ainda que $d_{2}: E_{2}^{p, q} \rightarrow E_{2}^{p+2, q+1}$ e portanto como pode ser visto no diagrama abaixo (onde os únicos $E_{2}^{p, q}$ não nulos são aqueles indicados explicitamente), $d_{2}$ é nula, o que garante que $E_{2}^{n, n}=E_{\infty}^{n, n}$.

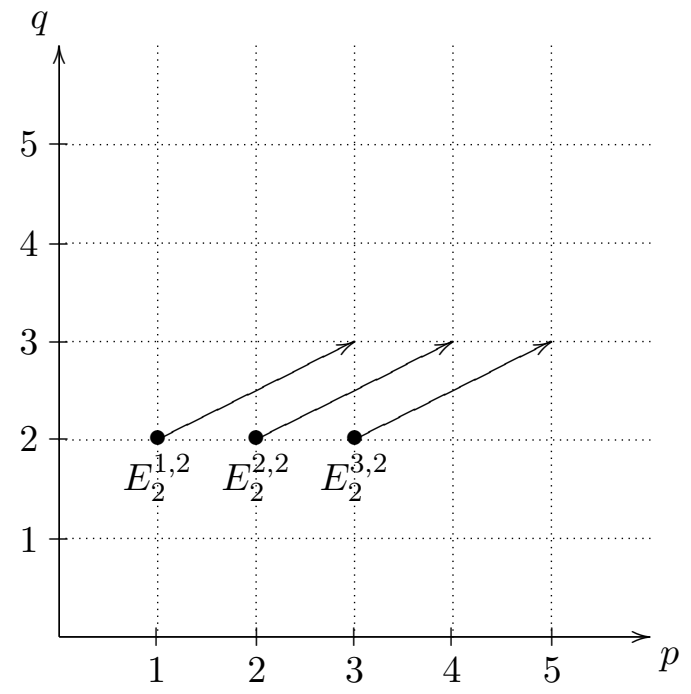

Como $E_{2}^{2,2}$ é o conjunto das classes de homotopia de $O$ a $(B G)_{2}=K(Z, 2)$ cuja projeção a $(B G)_{1}=*$ é homotópica a $p_{2} c_{0}$, segue de $E_{2}^{2,2}=H^{2}\left(O, \pi_{2}\left((B G), c_{0}\right)\right) \approx$ $H^{2}\left(O, \pi_{2}\left(B U_{2}\right)\right) \approx H^{2}(O, \mathbb{Z})$ que $\left[O,(B G)_{2}\right]=\mathbb{Z}_{2} \oplus \mathbb{Z}_{2}$, o que era de se esperar, uma vez que foi este o resultado obtido pelo método anterior. Observe que basta considerarmos a seqüência espectral de Larmore para a aplicação trivial $c_{0}$ porque $(B G)_{1}=*$.

De volta ao nosso objetivo para esta seção, o próximo passo é estudar o conjunto $\left[O,(B G)_{3}\right]$. No caso de $S^{3}$ basta calcularmos $E_{\infty}^{n, n}$ para qualquer $f: O \rightarrow(B G)_{3}$ porque existe uma única $p_{n} f$ possível, a aplicação trivial. Mas para o cálculo do mesmo $\left[O,(B G)_{3}\right]$, se $G=U_{2}$ ou $S_{3}$ temos que $p_{n} f$ pode assumir quatro possíveis valores já que $[O, K(Z, 2)]=H^{2}(O, Z) \approx \mathbb{Z}_{2} \oplus \mathbb{Z}_{2}$, o que deixa a situação completamente diferente.

Fixaremos então nossos estudos por enquanto na aplicação trivial e quando se 
fizer necessário dividiremos nossa atenção para os casos $G=S^{3}, U_{2}$ e $S_{3}$. Assim, seguimos com a construção de Larmore para obter

$$
E_{2}^{p, q}= \begin{cases}H^{p}\left(O, \pi_{q}\left(B G, c_{0}\right)\right), & \text { se } 2 \leq q \leq 3,1 \leq p \leq 4 \\ 0 & \text { caso contrário. }\end{cases}
$$

onde novamente $d_{2}: E_{2}^{p, q} \rightarrow E_{2}^{p+2, q+1}$ e no nível 2 da seqüência espectral, indicando apenas aqueles $E_{2}^{p, q}$ que são não-nulos e que se fazem necessários, temos:

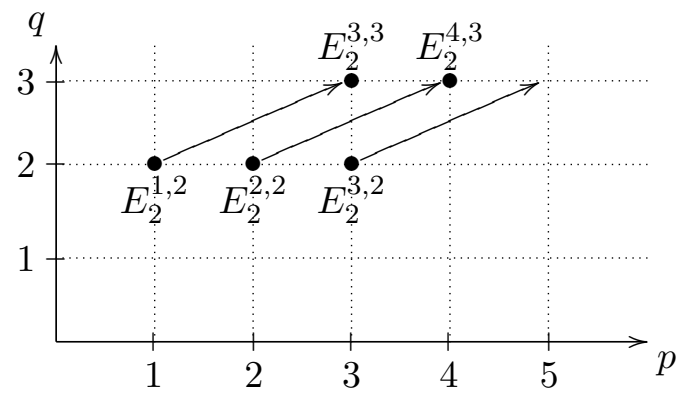

Segue imediatamente do diagrama que as únicas possibilidades que $d_{r}$ tem de não ser nula são os casos $d_{r}: E_{2}^{1,2} \rightarrow E_{2}^{3,3}$ e $d_{r}: E_{2}^{2,2} \rightarrow E_{2}^{4,3}$.

Se $G=U_{2}$, para $d_{r}: H^{1}\left(O, \pi_{2}(B G)\right)=H^{1}(O, \mathbb{Z}) \rightarrow H^{3}\left(O, \pi_{3}(B G)\right)=H^{3}(O, 0) \approx$ 0 , de onde tal aplicação é nula. Temos ainda $d_{r}: H^{2}\left(O, \pi_{2}\left(B U_{2}, c_{0}\right)\right)=H^{2}\left(O, \pi_{2}\left(B U_{2}\right)\right)=$ $H^{2}(O, \mathbb{Z}) \approx \mathbb{Z}_{2} \oplus \mathbb{Z}_{2} \rightarrow H^{4}\left(O, \pi_{3}\left(B U_{2}\right)\right)=H^{4}(O, 0) \approx 0$, e portanto esta aplicação também é nula.

Segue daí que $E_{\infty}^{3,3}=E_{2}^{3,3}$, e portanto $E_{\infty}^{3,3}=H^{3}\left(O, \pi_{3}\left(B U_{2}\right)\right)=H^{3}(O, 0) \approx$ 0 , ou seja, quando $G=U_{2}$, existe uma única classe de aplicações $[g]$ de $O$ até $(B G)_{3}$ tal que $p_{n} g$ é homotópica à aplicação trivial $p_{n} c_{0}$. Os casos não triviais são absolutamente idênticos a este porque o sheaf $\pi_{3}\left(\left(B U_{2}\right), f\right)$ continua sendo nulo, uma vez que $\pi_{3}(B G)$ é nulo.

No caso mais simples, quando $G=S^{3}$ vimos que $\left[O,(B G)_{2}\right]=H^{2}(O, Z) \approx 0$, ou seja, $p_{n} f$ é necessariamente trivial. Logo para qualquer aplicação $f: O \rightarrow(B G)_{3}$ temos $p_{n} f=p_{n} c_{0}$, e portanto a situação é análoga àquela do cálculo anterior. Daí, pelo que vimos antes, temos que existe uma única classe de aplicações de $O$ para $(B G)_{3}$ se $G=S^{3}$, ou seja, a única classe de 3 -fibrados sobre $O$ é a trivial.

Se $G=S O_{3}$, então $\pi_{2}\left(B S O_{3}\right) \approx \pi_{1}\left(S O_{3}\right) \approx \mathbb{Z}_{2}$ e $\pi_{3}\left(B S O_{3}\right) \approx \pi_{2}\left(S O_{3}\right) \approx$ 0 e daí segue no diagrama anterior que $d_{r}: H^{1}\left(O, \pi_{2}\left(B S O_{3}\right)\right)=H^{1}\left(O, \mathbb{Z}_{2}\right) \rightarrow$ $H^{3}\left(O, \pi_{3}\left(B S O_{3}\right)\right)=H^{3}(O, 0) \approx 0$, de onde a aplicação é nula, e também $d_{r}$ : $H^{2}\left(O, \pi_{2}\left(B S O^{3}, c_{0}\right)\right)=H^{2}\left(O, \mathbb{Z}_{2}\right) \rightarrow H^{4}(O, 0) \approx 0$ e daí $E_{\infty}^{3,3}=H^{3}\left(O, \pi_{3}\left(B S O^{3}\right)\right)=$ $H^{3}(O, 0) \approx 0$. A partir daí concluímos que neste caso também existe uma única classe 
de aplicações $g$ de $O$ até $(B G)_{3}$ tal que $p_{n} g$ é homotópica a $p_{n} c_{0}$. O caso não trivial é idêntico.

Note que todas as aplicações $p_{n} f$ se levantaram sem problemas até o nível seguinte da torre de Postnikov. Isso ocorre porque a aplicação diferencial $d_{r}$ em todos os casos foi trivial. Esta aplicação "mede" as obstruções, e sendo nula ela nos garante que não temos obstrução alguma, e portanto todas as aplicações $p_{n} f$ se levantam. Porém, nesta fase inicial do trabalho, foi utilizado outro método - mais trabalhoso - para conseguir os mesmos resultados. Este método pode ser conferido no apêndice.

Vamos resumir nosso exemplo até aqui com a seguinte classificação para fibrados vetoriais sobre $O$ :

$$
[O, B G]=\left[O,(B G)_{3}\right]= \begin{cases}0, & \text { se } G=S^{3} \\ \mathbb{Z}_{2} \oplus \mathbb{Z}_{2} & \text { se } G=U_{2} \text { ou } G=S O_{3} .\end{cases}
$$

Passemos então ao caso $G=O_{3}$. A primeira diferença é que como $\pi_{1}\left(\mathrm{BO}_{3}\right)=$ $\mathbb{Z}_{2} \neq 0$, então não temos equivalência entre $\left[O, B O_{3}\right]$ e $\left[O, B O_{3}\right]_{0}$ (o processo que apresentamos aqui calcula exatamente este último conjunto). Ainda em decorrência do fato de que $\pi_{1}\left(\mathrm{BO}_{3}\right)$ não é nulo, a princípio devemos trabalhar com coeficientes locais.

O passo seguinte é construir uma torre de Postnikov para $\mathrm{BO}_{3}$ :

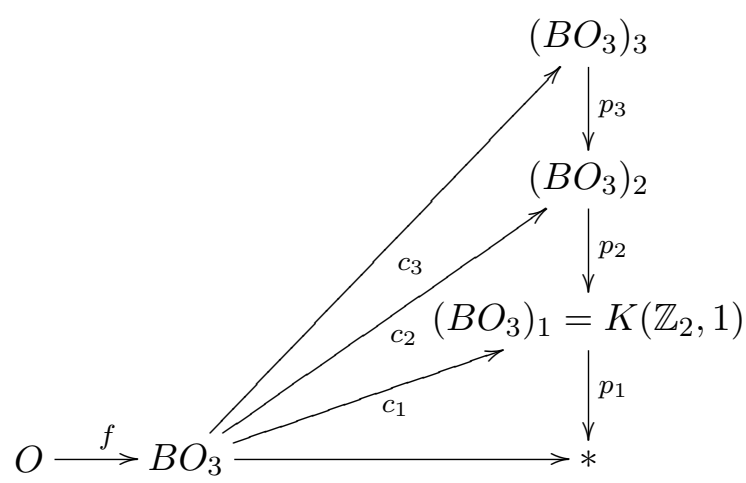

Agora observemos que a fibra de $p_{3}$ é um espaço de Eilenberg-McLane do tipo $\left[\pi_{3}\left(B O_{3}\right), 3\right]=[0,3]$, ou seja, a fibra é um ponto. Com isto, temos que $p_{3}$ induz um isomorfismo $\left[\mathrm{O},\left(\mathrm{BO}_{3}\right)_{3}\right] \approx\left[\mathrm{O},\left(\mathrm{BO}_{3}\right)_{2}\right]$ e portanto para classificarmos os 3-fibrados vetoriais reais sobre $\mathrm{O}$ basta encontrarmos $\left[\mathrm{O},\left(\mathrm{BO}_{3}\right)_{2}\right]$. (Uma breve análise mostra que poderíamos resolver os casos anteriores da mesma maneira.)

Note que $\left[O,\left(B O_{3}\right)_{1}\right]=\left[O, K\left(\mathbb{Z}_{2}, 1\right)\right]=H^{1}\left(O, \mathbb{Z}_{2}\right)=\mathbb{Z}_{2} \oplus \mathbb{Z}_{2}$. 
Seguindo em frente, primeiro vamos cuidar dos coeficientes, já que inicialmente teremos que trabalhar com coeficientes locais. Veja que $G_{n}\left(\left(B O_{3}\right)\right)$ é definido stalk por stalk sobre $\left(\mathrm{BO}_{3}\right)_{1}$, e como seu valor para cada ponto é sempre $\pi_{n}\left(\mathrm{BO}_{3}\right)$, temos que o stalk só pode ser trivial ou $\mathbb{Z}_{2}$. Se $\pi_{n}\left(B O_{3}\right)$ é trivial é claro que temos coeficientes globais tanto para $G_{n}\left(\left(B O_{3}\right)\right)$ como para seu pullback $\pi_{n}\left(B O_{3}, f\right)$, e se $\pi_{n}\left(B O_{3}\right)$ é igual a $\mathbb{Z}_{2}$, como este grupo tem um único isomorfismo, então existe uma maneira natural e canônica de se identificar cada stalk, ou seja, na prática estaremos trabalhando com coeficientes globais tanto ao lidarmos com $G_{n}\left(\left(B O_{3}\right)\right)$ como para seu pullback $\pi_{n}\left(B O_{3}, f\right)$, que é o que importa para nós. Outro detalhe importante é que como $\pi_{n}\left(B O_{3}, f\right)$ é independente de $f: O \rightarrow\left(B O_{3}\right)_{2}$, o processo a seguir não depende da classe de homotopia de $f$ - ou seja, o mesmo não se aplica apenas à aplicação trivial.

Tomemos então a aplicação constante $c_{0}: O \rightarrow\left(\mathrm{BO}_{3}\right)_{2}$, que é levantamento de $p_{n} c_{0}$. Como $n=2$, só podemos ter $q=2$ e $1 \leq p \leq 3$ e portanto os únicos elementos não-nulos da seqüência de Larmore são $E_{2}^{1,2}=H^{1}\left(O, \mathbb{Z}_{2}\right)=\mathbb{Z}_{2} \oplus \mathbb{Z}_{2}$, $E_{2}^{2,2}=H^{2}\left(O, \mathbb{Z}_{2}\right)=\mathbb{Z}_{2} \oplus \mathbb{Z}_{2}, E_{2}^{3,2}=H^{3}\left(O, \mathbb{Z}_{2}\right)=\mathbb{Z}_{2}$, como podemos ver no diagrama abaixo:

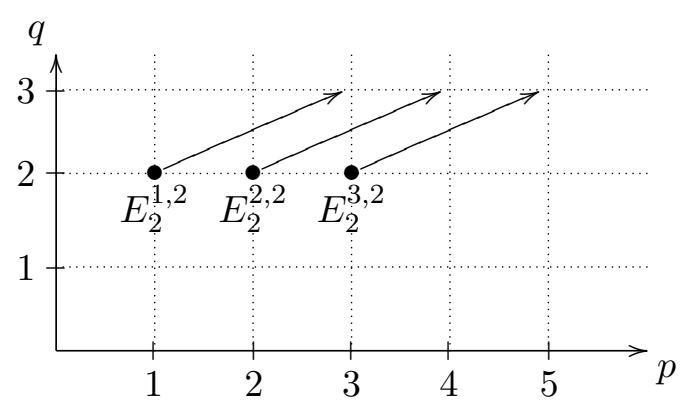

Isso quer dizer que $d_{r}$ é nula e portanto $E_{\infty}^{2,2}=E_{2}^{2,2}=\mathbb{Z}_{2} \oplus \mathbb{Z}_{2}$. Segue que temos quatro elementos na imagem inversa pela induzida de $p_{2}$ para cada classe em $\left[O,\left(\mathrm{BO}_{3}\right)_{1}\right]$, e com isto temos dezesseis 3-fibrados reais sobre $O$.

A seguir, vamos mostrar alguns exemplos do cálculo das diferenciais de uma seqüência espectral de Larmore. Para tanto, precisamos da definição e da observação a seguir - maiores detalhes podem ser encontrados em [11].

Definição 3.11. Seja $B$ qualquer espaço com ponto base, $p, q$ inteiros e $M, N$ grupos abelianos. Seja ainda $\operatorname{Map}(X, A ; B)=\{f: X \rightarrow B \mid f(A) \rightarrow *\}$ e $\mathcal{P}(Y)$ o conjunto das partes de $Y$. Nestes termos, entendemos por operação de aplicação de cohomologia (map-cohomology operation) de tipo $\{B, p, q, M, N\}$ uma coleção de funções 


$$
\theta: M a p(X, A ; B) \times H^{p}(X, A ; M) \rightarrow \mathcal{P}\left(H^{q}(X, A ; N)\right) .
$$

Existe uma tal função para cada $C W$-par $(X, A)$ e valem as seguintes propriedades:

1. Se $f \approx f^{\prime} r e l A$ então $\theta(f, x)=\theta\left(f^{\prime}, x\right)$.

2. Se $g:\left(X^{\prime}, A^{\prime}\right) \rightarrow(X, A)$ é qualquer aplicação, então $g^{*} \theta(f, x) \subset \theta\left(f g, g^{*} x\right)$.

Mais ainda, $\theta$ é dita homogênea se

3. para qualquer aplicação $f$, temos $0 \in \theta(f, 0)$.

Observação 3.7. Sejam $X, Y$ espaços e $2 \leq m<n$ inteiros tais que $\pi_{k}(Y)=0$ para todos os $k$ tais que $m<k<n$, e $f: X \rightarrow Y_{n}$ uma aplicação. Se o $k$ invariante $k^{n+1}$ de $Y$ (como definido em 3) é baseado na relação $\theta\left(\mathbb{1}, k^{m+1}\right)=0$, onde $\theta$ é uma aplicação de operação de cohomologia (map cohomology operation) como aquela de [10] e $\mathbb{1}: Y_{m-1} \rightarrow Y_{m-1}$ é a identidade. Nestas condições, para qualquer $x \in H^{m-1}\left(X, \pi_{m}(Y, f)\right)$ temos

$$
d_{r}(x)=s^{-2} \theta\left(p_{m-1}^{n} f P, s^{2} x\right),
$$

com $r=n-m+1$, onde $P: X \times S^{2} \rightarrow X$ é a projeção, $s^{2}: H^{*}\left(X, x_{0}\right) \rightarrow$ $H^{*+2}\left(X \times S^{2}, X \times * \cup x_{0} \times S^{2}\right)$ é a suspensão e $p_{m-1}^{n}=p_{m} \circ \ldots \circ p_{n}: Y_{n} \rightarrow Y_{m-1}$.

A tabela a seguir mostra os grupos de homotopia para os espaços classificantes dos grupos $O(1), O(2)$ e $O(3)$. Ela é parte daquela que pode ser encontrada na página 162 de [11].

\begin{tabular}{|l|c|c|c|}
\hline & $B O(1)$ & $B O(2)$ & $B O(3)$ \\
\hline$\pi_{1}$ & $\mathbb{Z} / 2$ & $\mathbb{Z} / 2$ & $\mathbb{Z} / 2$ \\
\hline$\pi_{2}$ & 0 & $\mathbb{Z}$ & $\mathbb{Z} / 2$ \\
\hline$\pi_{3}$ & 0 & 0 & 0 \\
\hline$\pi_{4}$ & 0 & 0 & $\mathbb{Z}$ \\
\hline$\pi_{5}$ & 0 & 0 & $\mathbb{Z} / 2$ \\
\hline$\pi_{6}$ & 0 & 0 & $\mathbb{Z} / 2$ \\
\hline
\end{tabular}


A partir desta tabela, podemos analisar os sheaves obtidos como em 2.1 a partir das torres de Postnikov de $B O(n), n=1,2,3$. É claro que os únicos casos em que poderemos ter sheaves não-globais são os casos $\pi_{2}(B O(2))$ e $\pi_{4}(B O(3))$. Para estes casos, basta observar que $\pi_{1}(B O(n))=\mathbb{Z} / 2$ age não-trivialmente em $\pi_{2}(B O(2))$ porque o primeiro $k$-invariante relativo de $B O(n) \rightarrow B O$ é

$$
k^{n+1}=\beta^{T}\left[\omega_{1}\right] \omega_{n} \in H^{n+1}\left(B O ; \mathbb{Z}^{T}\left[\omega_{1}\right]\right) .
$$

Observe que a redução mod 2 de $\Pi_{*} k^{n+1}$ deve ser necessariamente $\omega_{n+1}$, onde $\Pi$ é como definida em 2.1.1. $\mathbb{Z} / 2$ age trivialmente em $\pi_{4}(B O(3))$ porque $\mathbb{Z} / 2$ age trivialmente em $\pi_{4}(B O)$ e a aplicação $\mathbb{Z} \approx \pi_{4}(B O(3)) \rightarrow \pi_{4}(B O) \approx \mathbb{Z}$ é a multiplicação por dois (portanto é injetiva).

Agora podemos mostrar como calcular diferenciais $d_{r}=d_{r}^{f}$ para algumas aplicações $f: X \rightarrow Y_{k}$. No caso de $Y=B O(1)$, a tabela acima e a definição de cada $E_{2}^{p, q}$ da seqüência de Larmore nos garantem que todos os diferenciais são imediatamente nulos, o mesmo ocorrendo para o caso em que $Y=B O(2)$. Se $Y=B O(3)$, pelo mesmo motivo dos casos anteriores, todos os diferenciais são nulos quando $k<4$, sendo $f: X \rightarrow(B O(3))_{k}$. Porém, nem todos os diferenciais são nulos para $k \geq 4$.

Para entender o que ocorre aqui, vamos primeiramente ao caso $k=4$. Note que este $k$ corresponde ao índice $n$ na definição dos componentes da seqüência de Larmore. De acordo com esta definição, os únicos elementos não-nulos no nível dois da seqüência espectral $\left(E_{2}^{p, q}\right)$ são aqueles indicados no diagrama da figura 3.3, onde as setas indicam a ação de $d_{2}$.

Agora note que pela tabela acima, $\pi_{3}(B O(3))=0$, e este grupo é isomorfo ao stalk de cada sheaf $\pi_{3}(Y, f)$. Logo, este sheaf é nulo e é fácil ver no diagrama acima que isto anula $d_{2}$. A próxima diferencial, $d_{3}$, não é nula, como veremos a seguir.

Primeiramente, notemos que como $d_{2}$ é nula, a seqüência espectral é tal que $E_{2}^{p, q}=E_{3}^{p, q}$. Agora, como $d_{3}: E_{3}^{p, q} \rightarrow E_{3}^{p+3, q+2}$, segue que $d_{3}$ será não nula apenas na forma $d_{3}: H^{1}\left(X ; \pi_{2}(Y, f)\right) \rightarrow H^{4}\left(X ; \pi_{4}(Y, f)\right)$. De acordo com Larmore em [11] e com a observação 3.7 , temos que neste caso $d_{3}(x)=\beta\left(x^{3}+x \smile f^{*} \omega_{2}\right) \in H^{4}(X ; \mathbb{Z})$ para todo $x \in H^{1}(X ; \mathbb{Z} / 2)$, onde $\beta$ é o bockstein de $\mathbb{Z} \rightarrow \mathbb{Z} \rightarrow \mathbb{Z} / 2$.

Com os dados que temos agora podemos classificar todos os fibrados vetoriais reais de dimensão menor ou igual a três sobre $\mathbb{R} P^{k}, k \leq 4$ e indicar o método para $k=5$. As tabelas mostradas aqui podem ser encontradas em [11].

Começaremos com os fibrados vetoriais sobre $\mathbb{R} P^{1}=S^{1}$. O fibrados de dimensão um (fibrados por linha) são dados por $H^{1}\left(\mathbb{R} P^{1} ; \mathbb{Z} / 2\right)=\mathbb{Z} / 2$, o que significa que além do fibrado trivial temos um outro fibrado, que é na verdade o fibrado por linhas 
Figura 3.3:

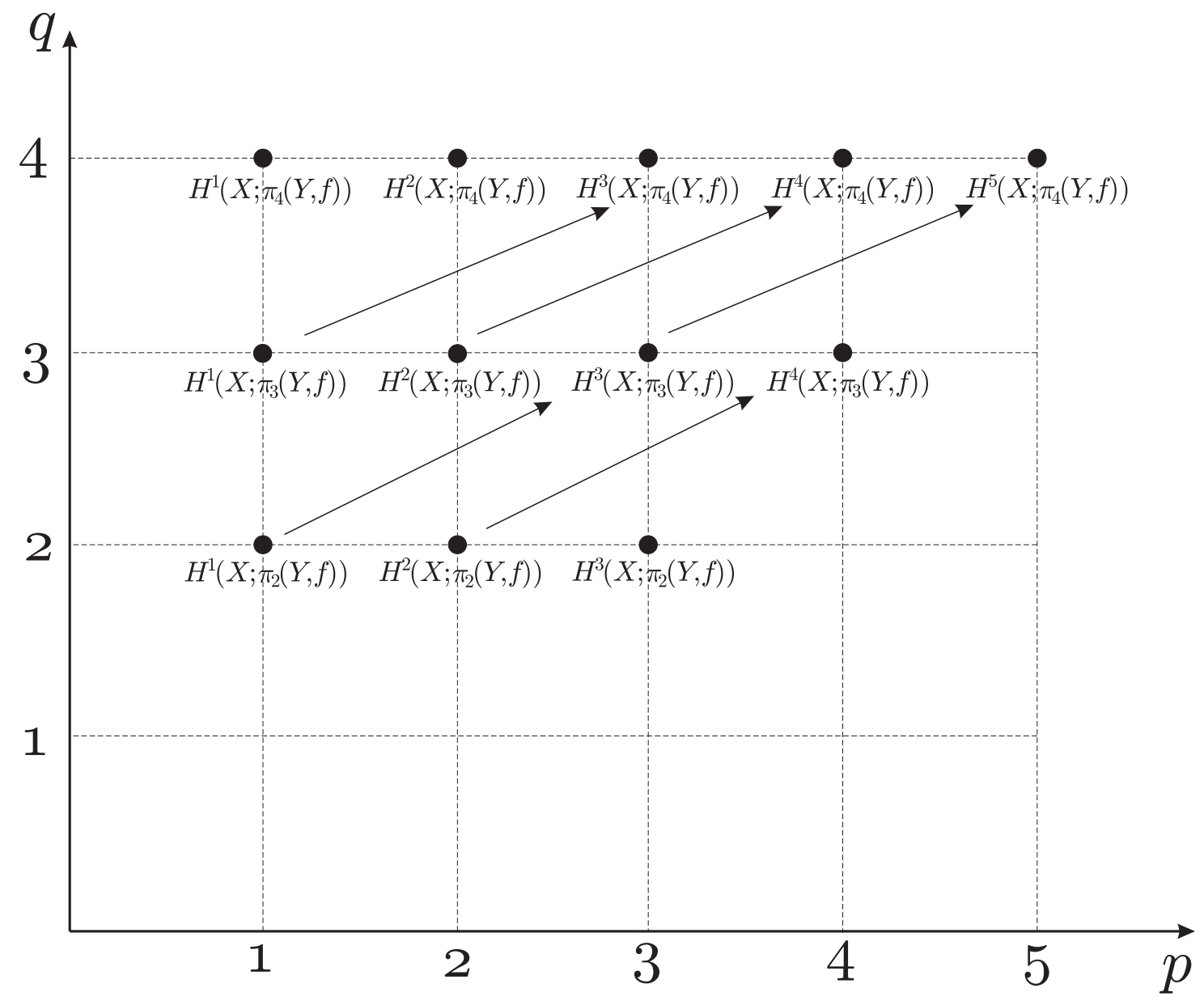

canônico visto em 1.2 , representado pelo gerador $u^{1}$ de $H^{1}\left(\mathbb{R} P^{1} ; \mathbb{Z} / 2\right)$. Vale observar que também podemos obter este resultado utilizando a seqüência de Larmore.

Agora precisamos discriminar os fibrados de dimensão dois sobre $\mathbb{R} P^{1}$. Até onde se sabe, não há uma forma imediata de se encontrar todos eles como acontece com os fibrados por linha, o que nos leva a usar o processo a seguir: Como $\mathbb{R} P^{1}$ tem dimensão 1 , segue de 1.1 que os fibrados de dimensão dois são dados por $\left[\mathbb{R} P^{1}, B O_{2}\right] \approx\left[\mathbb{R} P^{1},\left(B O_{2}\right)_{1}\right]$, onde $\left(B O_{2}\right)_{1}$ é o primeiro nível não-trivial da torre de Postnikov de $B O_{2}$. Agora, dada uma aplicação $f: \mathbb{R} P^{1} \rightarrow\left(B O_{2}\right)_{1}$, temos que a seqüência de Larmore para tal $f$ converge para ${ }^{f} E_{2}^{1,1}$ uma vez que pelo que vimos acima, a diferencial $d_{r}$ é sempre nula. Por definição temos $E_{2}^{1,1}=$ $H^{1}\left(\mathbb{R} P^{1} ; \pi_{1}\left(B O_{2}, f\right)\right)=H^{1}\left(\mathbb{R} P^{1} ; \mathbb{Z} / 2\right)=\mathbb{Z} / 2$, o que nos mostra que também exis- 
tem apenas dois fibrados, um trivial e outro não-trivial de dimensão dois sobre $\mathbb{R} P^{1}$.

Podemos então resumir os resultados para $\mathbb{R} P^{1}$ na seguinte tabela:

\begin{tabular}{|c|c|}
\hline \multicolumn{2}{|c|}{ Sobre $\mathbb{R} P^{1}$} \\
\hline $\operatorname{dim} 1$ & $\operatorname{dim} 2$ \\
\hline 1 & 1 \\
\hline $1+u^{1}$ & $1+u^{1}$ \\
\hline
\end{tabular}

Agora passaremos aos fibrados sobre $\mathbb{R} P^{2}$. Como no caso anterior, os fibrados de dimensão por linha estão em bijeção com $H^{1}\left(\mathbb{R} P^{2} ; \mathbb{Z} / 2\right)=\mathbb{Z} / 2$. Para determinar os fibrados de dimensão dois, como $\operatorname{dim}\left(\mathbb{R} P^{2}\right)=2$, basta para nós explicitar o conjunto $\left[\mathbb{R} P^{2},\left(B O_{2}\right)_{2}\right]$. Vamos então recorrer à torre de Postnikov de $B O_{1}$, que pode ser observada no diagrama abaixo:

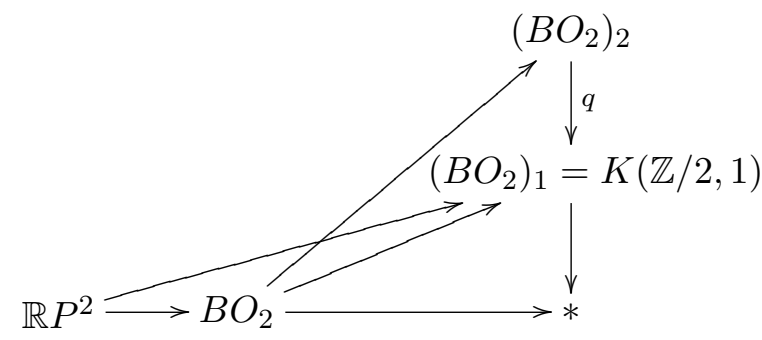

Veja que temos uma equivalência $\left[\mathbb{R} P^{2},\left(B O_{2}\right)_{1}\right]=\left[\mathbb{R} P^{2}, K(\mathbb{Z} / 2,1)\right]=H^{1}\left(\mathbb{R} P^{2}, \mathbb{Z} / 2\right)=$ $\mathbb{Z} / 2$ que nos garante que existem apenas duas classes de homotopia de aplicações de $\mathbb{R} P^{2}$ em $\left(B O_{2}\right)_{1}$ : a trivial e uma que chamaremos de $u^{1}$. Como todas estas aplicações se levantam, não existe obstrução alguma e portanto essas são classes que fazem o diagrama da torre de Postnikov comutar para, respectivamente, duas classes de aplicações $f, h: \mathbb{R} P^{2} \rightarrow\left(B O_{2}\right)_{2}$, utilizaremos a seqüência de Larmore para saber quantas classes $[g]: \mathbb{R} P^{2} \rightarrow\left(\mathrm{BO}_{2}\right)_{2}$ são levantamentos da classe trivial e da classe $u^{1}$.

Tomando-se a aplicação trivial $f$, como $d_{r}$ é nula, devemos ter ${ }^{f} E_{\infty}^{2,2}={ }^{f} E_{2}^{2,2}=$ $H^{2}\left(\mathbb{R} P^{2}, \pi_{2}\left(B O_{2}, f\right)\right)=H^{2}\left(\mathbb{R} P^{2}, \mathbb{Z}\right)$. É importantíssimo notar que como estamos considerando $f$ trivial, não há torção no sheaf $\pi_{2}\left(B O_{2}, f\right)$ e portanto estamos tratando de coeficientes globais, de onde ${ }^{f} E_{\infty}^{2,2}=\mathbb{Z} / 2$, gerado por $u^{2}$.

Já no caso em que tomamos a aplicação $h: \mathbb{R} P^{2} \rightarrow\left(B O_{2}\right)_{2}$, teremos ${ }^{h} E_{\infty}^{2,2}={ }^{h}$ $E_{2}^{2,2}=H^{2}\left(\mathbb{R} P^{2}, \pi_{2}\left(B O_{2}, h\right)\right)=H^{2}\left(\mathbb{R} P^{2}, \mathbb{Z}^{T}[u]\right)$, onde $\left.\mathbb{Z}^{T}[u]\right)$ é o sheaf de coeficientes torcidos definido em 2.1, obtido através do pullback pela aplicação $h$. De acordo com os resultados em 2.2 , temos que $H^{2}\left(\mathbb{R} P^{2}, \mathbb{Z}^{T}[u]\right)=\mathbb{Z}$, gerado pela "top class" $t\left(\mathbb{R} P^{2}\right)$. 
Passemos então ao caso dos fibrados de dimensão três sobre $\mathbb{R} P^{2}$, o que nos leva a trabalhar com $\mathrm{BO}_{3}$. Devido à dimensão dois de $\mathbb{R} P^{2}$, basta trabalharmos até o nível dois da torre de Postnikov de $\mathrm{BO}_{3}$, como visto abaixo:

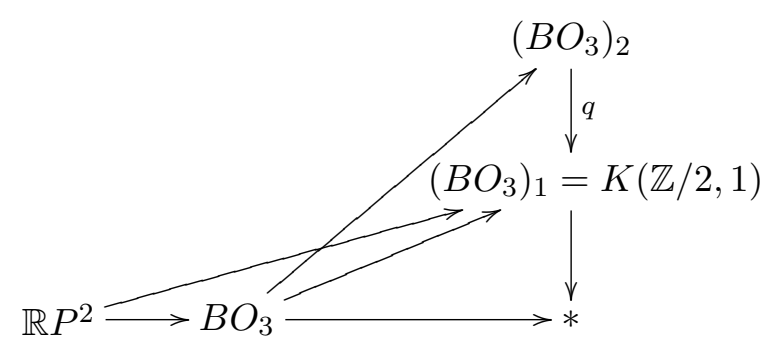

A situação é idêntica ao caso anterior: temos duas classes de aplicações de $\mathbb{R} P^{2}$ em $\left(B O_{3}\right)_{1}$, uma trivial e outra não-trivial e devemos então novamente considerar levantamentos $[f]$ e $[h]$ para cada uma destas e a partir daí, como $d_{r}$ é nula, calcularmos ${ }^{f} E_{\infty}^{2,2}={ }^{f} E_{2}^{2,2}=H^{2}\left(\mathbb{R} P^{2}, \pi_{2}\left(B O_{3}, f\right)\right)$ e também ${ }^{h} E_{\infty}^{2,2}={ }^{h} E_{2}^{2,2}=$ $H^{2}\left(\mathbb{R} P^{2}, \pi_{2}\left(B O_{3}, h\right)\right)$. A diferença aqui é que tanto para $f$ quanto para $h$ teremos um sheaf de coeficientes globais, uma vez que $\pi_{2}\left(B O_{3}\right)=\mathbb{Z} / 2$. Segue daqui que ${ }^{f} E_{\infty}^{2,2}={ }^{h} E_{\infty}^{2,2}=\mathbb{Z} / 2$, gerado por $u^{2}$.

Podemos agora resumir os resultados para $\mathbb{R} P^{2}$ na seguinte tabela:

\begin{tabular}{|c|c|c|}
\hline \multicolumn{3}{|c|}{ Sobre $\mathbb{R} P^{2}$} \\
\hline $\operatorname{dim} 1$ & $\operatorname{dim} 2$ & $\operatorname{dim} 3$ \\
\hline \multirow{2}{*}{1} & 1 & 1 \\
\cline { 2 - 3 } & $1+u^{2}$ & $1+u^{2}$ \\
\hline \multirow{2}{*}{$1+u^{1}$} & $1+u^{1}+k t\left(\mathbb{R} P^{2}\right), k \in \mathbb{Z}$ & $1+u^{1}$ \\
\cline { 2 - 3 } & & $1+u^{1}+u^{2}$ \\
\hline
\end{tabular}

Consideraremos a partir daqui os fibrados vetoriais sobre $\mathbb{R} P^{3}$. Mais uma vez, de maneira idêntica às anteriores, temos dois fibrados por linha, um trivial e outro indicado por $u^{1}$, gerador de $H^{1}\left(\mathbb{R} P^{3} ; \mathbb{Z} / 2\right)$. Os fibrados de dimensão dois são dados por $\left[\mathbb{R} P^{3}, B O_{2}\right] \approx\left[\mathbb{R} P^{3},\left(B O_{2}\right)_{3}\right]$, devido à dimensão três de $\mathbb{R} P^{3}$. Vejamos a torre de Postnikov até dimensão 3 para $\mathrm{BO}_{2}$ : 


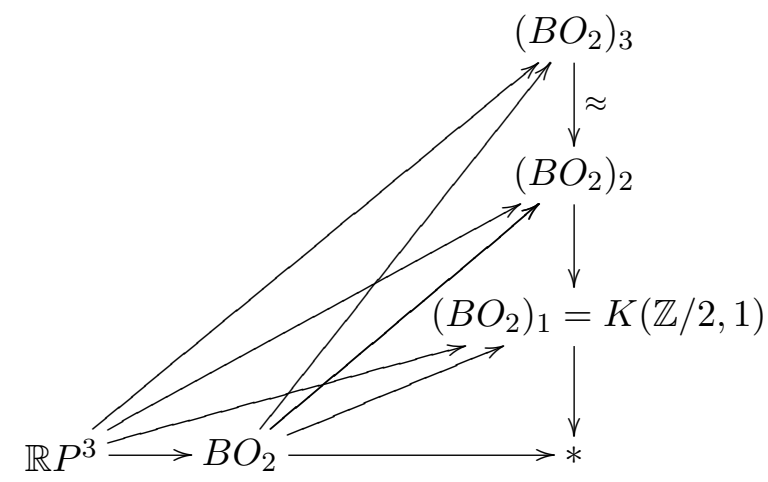

Como $\pi_{3}\left(B O_{n}\right)=0$, segue que temos um isomorfismo entre o segundo e o terceiro níveis da torre de Postnikov. É claro que a partir daí, determinar o conjunto $\left[\mathbb{R} P^{3},\left(B O_{2}\right)_{3}\right]$ é a mesma coisa que determinar o conjunto $\left[\mathbb{R} P^{3},\left(B O_{2}\right)_{2}\right]$. Procederemos então como no caso anterior: as classes de aplicações de $\mathbb{R} P^{3}$ em $\left(B O_{2}\right)_{1}$ são dadas por $H^{1}\left(\mathbb{R} P^{3} ; \mathbb{Z} / 2\right)=\mathbb{Z} / 2$, gerado por $u^{1}$. Chamando de $[f]$ um levantamento da classe trivial e de $[h]$ um levantamento da classe de $h$, temos que ${ }^{f} E_{\infty}^{2,2}={ }^{f} E_{2}^{2,2}$, assim como ${ }^{h} E_{\infty}^{2,2}={ }^{h} E_{2}^{2,2}$ pois $d_{r}$ é nula sempre que consideramos uma aplicação $f$ saindo de $\mathbb{R} P^{3}$ e chegando num nível estritamente menor que 4 na torre de Postnikov de $\mathrm{BO}_{2}$, o que é o caso.

Agora, ${ }^{f} E_{2}^{2,2}=H^{2}\left(\mathbb{R} P^{3} ; \pi_{2}\left(B O_{2}, f\right)\right)=H^{2}\left(\mathbb{R} P^{3} ; \mathbb{Z}\right)=\mathbb{Z} / 2$ gerado por $u^{2}$, onde os coeficientes são globais porque a $f$ é trivial.

Se tomarmos a aplicação não-nula $h$, teremos um sheaf de coeficientes torcidos e portanto ${ }^{h} E_{2}^{2,2}=H^{2}\left(\mathbb{R} P^{3} ; \pi_{2}\left(B O_{2}, h\right)\right)=H^{2}\left(\mathbb{R} P^{3} ; \mathbb{Z}^{T}[u]\right)=0$, de acordo com o que fizemos em 2.2.

O caso da dimensão 3 para $\mathbb{R} P^{3}$ é idêntico a este, com a exceção de que devido ao fato de que $\pi_{2}\left(B O_{3}\right)=\mathbb{Z} / 2$, devemos ter todos os sheaves com stalk $\mathbb{Z} / 2$, o que implica que todos eles terão coeficientes globais. Logo teremos, usando a mesma notação dos casos anteriores, ${ }^{f} E_{\infty}^{2,2}={ }^{h} E_{\infty}^{2,2}=\mathbb{Z} / 2$, gerado por $u^{2}$.

Como observação final desta parte do exemplo, vale notar que o caso dos fibrados de dimensão quatro é absolutamente análogo a este de dimensão três, como pode ser visto em [11]. Os resultados para $\mathbb{R} P^{3}$ podem ser resumidos na tabela a seguir: 


\begin{tabular}{|c|c|c|}
\hline \multicolumn{3}{|c|}{ Sobre $\mathbb{R} P^{3}$} \\
\hline $\operatorname{dim} 1$ & $\operatorname{dim} 2$ & $\operatorname{dim} 3$ \\
\hline \multirow{2}{*}{1} & 1 & 1 \\
\cline { 2 - 3 } & $1+u^{2}$ & $1+u^{2}$ \\
\hline \multirow{2}{*}{$1+u^{1}$} & $1+u^{1}$ & $1+u^{1}$ \\
\cline { 3 - 3 } & & $1+u^{1}+u^{2}$ \\
\hline
\end{tabular}

Passemos agora ao caso dos fibrados vetoriais sobre $\mathbb{R} P^{4}$. Analisemos então a torre de Posnikov para $\mathrm{BO}_{2}$ a fim de encontrarmos os fibrados vetoriais de dimensão dois:

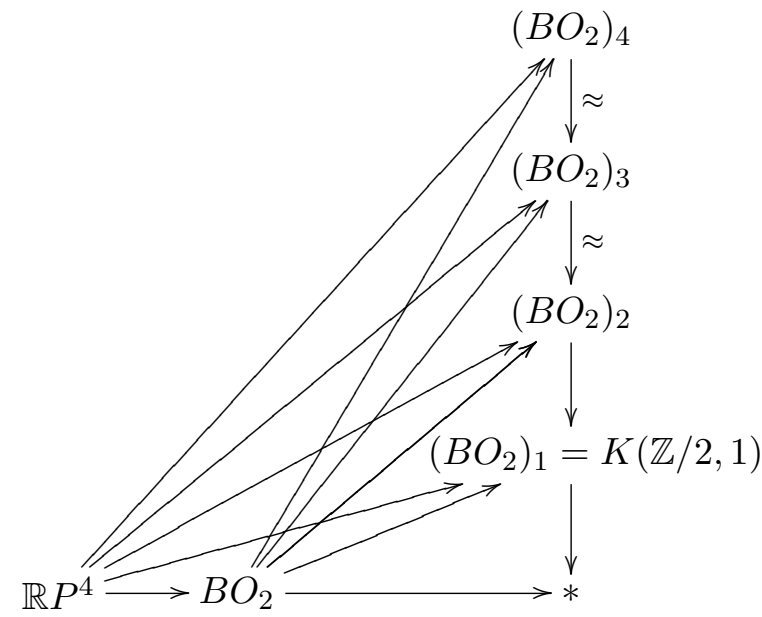

Decorre do fato de que $\pi_{4}\left(B_{2}\right)=\pi_{3}\left(B O_{2}\right)=0$ que calcular as classes de aplicações em $\left[\mathbb{R} P^{4}, B \mathrm{O}_{2}\right]$ é equivalente a calcular as classes de aplicações em $\left[\mathbb{R} P^{4},\left(B O_{2}\right)_{2}\right]$. Analogamente ao que fizemos anteriormente, podemos encontrar duas classes de aplicações, uma $[f]$ trivial e uma $[h]$ não trivial de $\mathbb{R} P^{4}$ em $\left(B O_{2}\right)_{1}$. De acordo com o que fizemos antes, os fibrados de dimensão dois sobre $\mathbb{R} P^{4}$ são então dados por $H^{2}\left(\mathbb{R} P^{4}, \pi_{2}\left(B O_{2}, f\right)\right)=\mathbb{Z} / 2$ gerado por $u^{2}$ e por $H^{2}\left(\mathbb{R} P^{4}, \pi_{2}\left(B O_{2}, h\right)\right)=0$, onde no último grupo de cohomologia o sheaf é de coeficientes torcidos.

Para os fibrados de dimensão três a torre de Postnikov é a seguinte: 


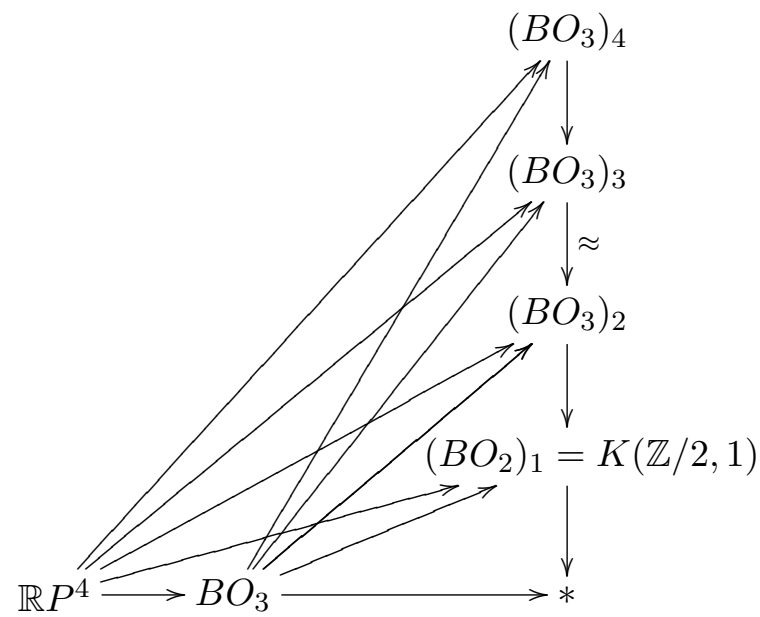

Aqui a única equivalência é entre $\left[\mathbb{R} P^{4},\left(\mathrm{BO}_{3}\right)_{2}\right]$ e $\left[\mathbb{R} P^{4},\left(\mathrm{BO}_{3}\right)_{3}\right]$, sendo que este conjunto é determinado de modo inteiramente análogo ao que vimos nesta mesma seção para os casos anteriores. A grande diferença deste caso para os anteriores é a diferencial $d_{3}$, que não é trivial. Vejamos como este fato altera nossa forma de trabalho.

As classes de aplicações de $\mathbb{R} P^{4}$ em $\left(\mathrm{BO}_{3}\right)_{3}$ são quatro, obtidas de forma idêntica ao que já vimos antes, e serão indicadas por $[f],\left[f+u^{2}\right],[h],\left[h+u^{2}\right]$ ou, se preciso, 1,1+ $u^{2}, 1+u^{1}, 1+u^{1}+u^{2}$ (note que essa notação é um tipo de "licença poética" porque neste nível da torre de Postnikov ainda não estamos lidando com os fibrados procurados, que serão identificados com uma notação semelhante a esta.) Tomemos o levantamento da classe trivial, que por abuso de notação chamaremos de $[f]$ e vejamos sua seqüência de Larmore: pelo que vimos antes, a diferencial $d_{2}$ é nula, o que implica que os níveis dois e três da seqüência espectral são iguais. A diferencial $d_{3}$ é não-nula quando $d_{3}: H^{1}\left(\mathbb{R} P^{4} ; \pi_{2}\left(B O_{3}, f\right)\right)=$ $H^{1}\left(\mathbb{R} P^{4} ; \mathbb{Z} / 2\right)=\mathbb{Z} / 2 \rightarrow H^{4}\left(\mathbb{R} P^{4} ; \pi_{4}\left(B O_{3}, f\right)\right)=H^{4}\left(\mathbb{R} P^{4} ; \mathbb{Z}\right)=\mathbb{Z} / 2$, onde é igual a $d_{3}(x)=\beta\left(x^{3}+x \smile f^{*} \omega_{2}\right)$. Note que $f^{*} \omega_{2}$ é o pullback da classe $\omega_{2}$ via $f$, e portanto basta observarmos o elemento correspondente na notação, como no caso da aplicação trivial o elemento correspondente é nulo, segue que $f^{*} \omega_{2}$ é nulo - ou seja, aqui teremos $d_{3}(x)=\beta\left(x^{3}\right)$. Como o bockstein não é nulo, temos que a $d_{3}$ "mata" o elemento não-trivial em sua imagem, o que nos leva a concluir que no próximo nível da seqüência espectral teremos $E_{4}^{4,4}=0$. Como $d_{r}$ é trivial para $r \geq 4$, temos $E_{\infty}^{4,4}=E_{4}^{4,4}=0$.

Agora o caso da segunda aplicação $\left[f+u^{2}\right]$, também representada por $1+$ $u^{2}$. Uma vez que o elemento $u^{2}$ corresponde ao pullback $f^{*} \omega_{2}$, a diferencial $d_{3}$ é dada por $d_{3}(x)=\beta\left(x^{3}+x \smile x^{2}\right)=\beta\left(x^{3}+x^{3}\right)=\beta(0)=0$, uma vez que 
$x \in H^{1}\left(\mathbb{R} P^{4} ; \pi_{2}\left(B O_{3}, g\right)\right)=\mathbb{Z} / 2$ independente da aplicação $g$ considerada por que $\pi_{2}\left(B O_{3}\right)=\mathbb{Z} / 2$. Veja ainda que $H^{4}\left(\mathbb{R} P^{4} ; \pi_{4}\left(B O_{3}, g\right)\right)=\mathbb{Z} / 2$ independente da aplicação $g$ por que como vimos antes, $\mathbb{Z} / 2$ age trivialmente em $\pi_{4}\left(B O_{3}\right)$. Com isto, a imagem de $d_{3}$ neste caso é nula, de onde $E_{\infty}^{4,4}=E_{4}^{4,4}=E_{3}^{4,4}=H^{4}\left(\mathbb{R}^{4} ; \pi_{4}\left(B O_{3}, g\right)\right)=$ $\mathbb{Z} / 2$

O caso da classe $[h]$ também representada por $1+u^{1}$ é idêntico ao caso de $f$ e o caso de $\left[h+u^{2}\right]$, também representada por $1+u^{1}+u^{2}$, é idêntico ao de $f+u^{2}$. Assim, podemos resumir os resultados na seguinte tabela:

\begin{tabular}{|c|c|c|}
\hline \multicolumn{3}{|c|}{ Sobre $\mathbb{R} P^{4}$} \\
\hline $\operatorname{dim} 1$ & $\operatorname{dim} 2$ & $\operatorname{dim} 3$ \\
\hline \multirow{2}{*}{1} & 1 & 1 \\
\cline { 2 - 3 } & $1+u^{2}$ & $1+u^{2}$ \\
\hline \multirow{2}{*}{$1+u^{1}$} & $1+u^{1}+u^{2}$ & $1+u^{1}$ \\
\cline { 2 - 3 } & & $1+u^{1}+u^{2}$ \\
\hline
\end{tabular}

Vale acrescentar que os fibrados vetoriais de dimensões quatro e cinco sobre $\mathbb{R} P^{4}$ também podem ser contados com o método acima, mas com complicações maiores devidas, por exemplo, ao fato de que $\pi_{4}\left(B O_{4}\right)=\mathbb{Z} \oplus \mathbb{Z}$ com ação não trivial por parte de $\mathbb{Z} / 2$. Os fibrados sobre $\mathbb{R} P^{5}$ de dimensão dois e três são obtidos de maneira inteiramente análoga ao que fizemos até aqui, enquanto aqueles de dimensão quatro, cinco e seis necessitam de maiores informações sobre as diferenciais $d_{r}$. A tabela completa, porém sem tais detalhes, pode ser encontrada em [11]. 


\section{Capítulo 4}

\section{Resultados gerais obtidos para}

\section{classificação de fibrados}

Neste capítulo nosso objetivo é explicitar os resultados obtidos sobre classificação de fibrados, para então utilizá-los no capítulo seguinte para enumerar fibrados sobre as "spherical space forms". Tais resultados tem uma natureza mais geral do que aqueles do capítulo seguinte, uma vez que aqui os grupos estruturais abordados podem ser, inicialmente, quaisquer grupos de Lie conexos e mais à frente quaisquer grupos de Lie compactos que possuem certa propriedade bastante comum a estes grupos. Além disso, o fato de que a variedade fechada $M$ trabalhada neste capítulo não é necessariamente uma spherical space form torna a abrangência dos resultados consideravelmente maior. O símbolo "=" quando usado entre dois conjuntos quer dizer bijeção. Primeiro trataremos do caso onde o grupo estrutural é conexo:

Teorema 4.1. Seja $G$ um grupo de Lie conexo e $M=M^{n}$ uma variedade fechada de dimensão n menor ou igual a três. Então o conjunto das classes de equivalência de $G$-fibrados principais sobre $M$, denotado por $\mathcal{B}(M, G)$ é dado por

$$
\begin{aligned}
\mathcal{B}\left(M^{1}, G\right) & =0 \\
\mathcal{B}\left(M^{2}, G\right)=\mathcal{B}\left(M^{3}, G\right) & =H^{2}\left(M ; \pi_{1}(G)\right)
\end{aligned}
$$

Demonstração. Como vimos no capítulo inicial, temos uma bijeção entre $\mathcal{B}(M, G) \mathrm{e}$ $[M, B G]$, onde $B G$ é o espaço de classificação de $G$ e $[M, B G]$ denota o conjunto de classes de homotopia de aplicações. Seguindo a notação adotada na definição 3.1, de acordo com [25], se $M$ tem dimensão $n$ então temos que $p_{m}^{*}$ é uma bijeção entre 
$[M, B G]$ e $\left[M, B G_{m}\right]$, onde $B G_{m}$ denota o espaço que ocupa o n-ésimo nível na torre de Postnikov de $B G$. Observemos que estes dois fatos já são são suficientes para a prova do presente teorema (e também do teorema 4.2) em dimensão 1.

Agora note que se $M$ tem dimensão 2 ou 3 e $p_{2}^{*}$ é como definida em 3.1, então também temos uma bijeção entre $[M, B G]$ e $\left[M,(B G)_{2}\right]$. Isto acontece porque todo grupo de Lie conexo tem o mesmo tipo de homotopia de um subgrupo próprio compacto, porém todo grupo de Lie conexo compacto tem $\pi_{2}$ nulo, daí segue que $\pi_{2}(G)=0$. Com isto, a fibra relativa à fibração $q_{3}$ na torre de Postnikov é apenas um ponto, logo temos a bijeção requerida.

Note também que $\pi_{1}(G)=\pi_{2}(B G)$ é abeliano, o que justifica a bijeção $\left[M,(B G)_{2}\right]=$ $\left[M,(B G)_{2}\right]_{0}$. Sabemos ainda que para todo $G$ conexo a primeira fibração não trivial na torre de Postnikov de $B G$ tem espaço total $(B G)_{2}$ e fibra um espaço de Eilenberg-MacLane $K\left(\pi_{1}(G), 2\right)$, a partir daí a teoria de obstrução nos garante que $\left[M,(B G)_{2}\right]=\left[M,(B G)_{2}\right]_{0}$ está em bijeção com $H^{2}(M, \pi(G))$. Estas bijeções provam o teorema para as dimensões 2 e 3 .

O próximo passo é extender este resultado para o caso de um grupo não conexo $G$, uma vez que vários exemplos importantes de fibrados provém de grupos estruturais não conexos. Trabalharemos então com grupos compactos, que nos permitem encontrar um resultado que cobre os casos mais importantes, como os fibrados vetoriais reais.

Antes de apresentar os resultados, vamos recordar alguns fatos de teoria de grupos de Lie que podem ser vistos em maiores detalhes em [3] ou em [15]. Comecemos pela componente conexa $G_{0}$ que contém a identidade. Ela é um subgrupo normal fechado e o grupo quociente $G / G_{0}$ é um grupo finito isomorfo a $\pi_{0}(G)$. Mais ainda, existe uma seqüência exata de grupos de Lie e homomorfismos contínuos

$$
0 \longrightarrow G_{0} \stackrel{i_{0}}{\longrightarrow} G \stackrel{p_{0}}{\longrightarrow} G / G_{0} \longrightarrow 0 .
$$

Suposição 4.1. Daqui até o final desta seção, estaremos supondo que esta seqüência exata acima cinde através de um homomorfismo continuo $s: G / G_{0} \rightarrow G$

Note que a classe dos grupos de Lie que satisfazem tal suposição é grande e contém todos os casos mais importantes. Assim, segue que $G$, como grupo topológico, é isomorfo ao produto semidireto $G / G_{0} \ltimes_{\alpha} G_{0}$, para algum homomorfismo $\alpha: G / G_{0} \rightarrow$ Aut $(G)$.

Daqui em diante vamos denotar os elementos de $G / G_{0}$ como elementos de $G$ usando a identificação fornecida por $s$. Assim, $G$ é o produto semidireto $G / G_{0} \ltimes_{\alpha} G_{0}$ com multiplicação dada por $(a, g)(b, h)=(a b, \alpha(b)(g) h)$, e ainda, a ação à esquerda 
de $G / G_{0}$ em $G$ definida por $(a, g) \mapsto \alpha(a)(g)$ é contínua. Nestas condições, podemos utilizar a Proposição 1 de [16]:

Proposição 26. O espaço de classificação $B G$ é o espaço total de um $G / G_{0}$-fibrado sobre $B\left(G / G_{0}\right)$ com fibra $B G_{0}$ e projeção $B p_{0}: B G \rightarrow B\left(G / G_{0}\right)$. Mais ainda, a aplicação que cinde (splitting map) s induz um cross section $B s: B\left(G / G_{0}\right) \rightarrow B G$.

Demonstração. Faremos uso aqui do fato de que para um grupo topológico arbitrário $G$ existe um $G$-fibrado principal universal $\xi_{G}=(E G, p G, B G)$ - a construção de tal estrutura pode ser vista em [18] e [17]. Também assumiremos trabalhar numa categoria conveniente de espaços, assim como feito em [17] para termos garantida a continuidade das funções consideradas.

Note que os homomorfismos contínuos

$$
\begin{aligned}
& s: \underset{a}{G}\left(G_{Q}, \overrightarrow{1_{G}}\right), \\
& \left.\stackrel{\ell}{g}: G_{Q} \underset{1_{\Gamma}}{\longrightarrow}, G\right) \\
& p_{o}:(a, g) \mapsto G / G_{0},
\end{aligned}
$$

induzem morfismos

$$
\begin{aligned}
& E s: E\left(G / G_{0}\right) \rightarrow E G, \quad E \ell: E G_{0} \rightarrow E G, \quad E p_{o}: E G \rightarrow E\left(G / G_{0}\right) \\
& B s: B\left(G / G_{0}\right) \rightarrow B G, \quad B \ell: B G_{0} \rightarrow B G, \quad B p_{o}: B G \rightarrow B\left(G / G_{0}\right) .
\end{aligned}
$$

Mais ainda, observe que existe uma ação

$$
\begin{gathered}
A: E G_{0} \times G / G_{0} \rightarrow E G_{0} \\
(x, a) \mapsto a^{-1} x a
\end{gathered}
$$

que pode ser passada para $B G_{0}$ pela comutatividade do seguinte diagrama:

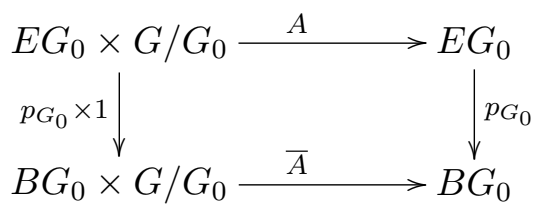

Estas ações geram espaços quocientes $E\left(G / G_{0}\right) \times_{\Gamma} E G_{0}$ e $E\left(G / G_{0}\right) \times_{\Gamma} B G_{0}$ com suas correspondentes ações quocientes $\pi: E\left(G / G_{0}\right) \times E G_{0} \rightarrow E\left(G / G_{0}\right) \times_{\Gamma} E G_{0}$, $\pi^{\prime}: E\left(G / G_{0}\right) \times B G_{0} \rightarrow E\left(G / G_{0}\right) \times_{\Gamma} B G_{0}$

O homomorfimo de grupos

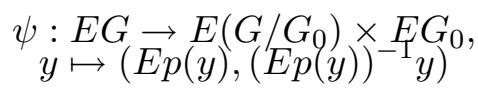


é na verdade um isomorfismo com inversa

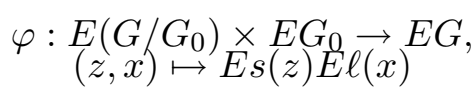

como podemos verificar:

$\varphi \psi(y)=\varphi\left(E p(y),(E p(y))^{-1} y\right)=E p(y)(E p(y))^{-1} y=y$, enquanto por outro lado temos

$$
\begin{gathered}
\psi \varphi(z, x)=\psi(E s(z) E \ell(x))=\left(E p(E s(z) E \ell(x)),\left((E p(E s(z) E \ell(x)))^{-1}(E s(z) E \ell(x))=\right.\right. \\
=(E(p s)(z) E(p \ell)(x), \ldots)=\left(z, z^{-1} z x\right)=(z, x) .
\end{gathered}
$$

Computações rotineiras permitem ver que as aplicações $\psi$ e $\varphi$ passam para os espaços quocientes e que compostas resultam na identidade $1_{B \Gamma}$.

Agora estamos em condições de enunciar e provar nosso próximo teorema:

Teorema 4.2. Seja $G$ um grupo de Lie compacto satisfazendo a suposição 4.1, e $M$ uma variedade fechada de dimensão menor ou igual a três. Então

$$
\begin{aligned}
& \mathcal{B}\left(M^{1}, G\right)=\frac{\operatorname{Hom}\left(\pi_{1}\left(M^{1}\right), \pi_{0}(G)\right)}{P \pi_{0}(G)} \\
& \mathcal{B}\left(M^{2,3}, G\right)=\bigsqcup_{\phi \in \frac{H o m\left(\pi_{1}(M), \pi_{0}(G)\right)}{P \pi_{0}(G)}} \frac{H^{2}\left(M ; \pi_{1}\left(G, u_{\phi}\right)\right)}{\pi_{0}(G)},
\end{aligned}
$$

onde $P \pi_{0}(G)=\frac{\pi_{0}(G)}{Z \pi_{0}(G)}$.

Demonstração. Utilizaremos a seguinte torre de Postnikov para $B G$, onde $B\left(G / G_{0}\right)=$ $K\left(\pi_{0}(G), 1\right)$ :

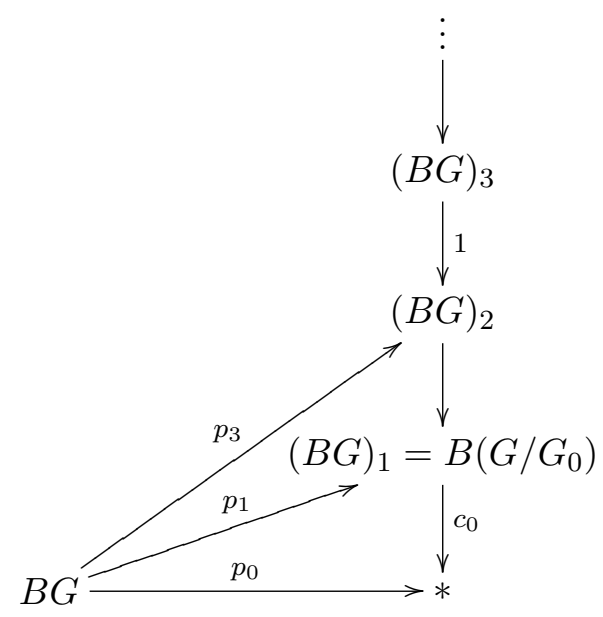


A partir da existência de cross-section garantida pela proposição 26 podemos concluir que $\left(B p_{0}\right)_{*}$ é sobrejetora e portanto temos então uma seqüência exata de conjuntos

$$
\left[M, B G_{0}\right] \stackrel{\left(B i_{0}\right)_{*}}{\longrightarrow}[M, B G] \stackrel{\left(B p_{0}\right)_{*}}{\longrightarrow}\left[M, B\left(G / G_{0}\right)\right] \longrightarrow 0 .
$$

Trataremos primeiro do caso onde a dimensão de $M$ é 1 , onde

$$
\begin{gathered}
{\left[M^{1}, B G\right]=\left[M^{1},(B G)_{1}\right]=\left[M^{1}, B\left(G / G_{0}\right)\right]=\frac{\left[M^{1}, B\left(G / G_{0}\right)\right]_{0}}{\pi_{0}(G)}=} \\
=\frac{\operatorname{Hom}\left(\pi_{1}\left(M^{1}\right), \pi_{0}(G)\right)}{\pi_{0}(G)},
\end{gathered}
$$

e a ação é dada por $(\phi, \alpha)(x) \mapsto \phi^{\alpha}(x)=\alpha^{-1} \phi(x) \alpha$, o que nos permite considerar apenas automorfismos internos - e isso conclui a prova para dimensão 1.

Agora tomemos $M$ de dimensão 2 ou 3. Temos

$$
\left[M, B\left(G / G_{0}\right)\right]_{0}=\left[M, K\left(\pi_{0}(G), 1\right)\right]_{0}=\operatorname{Hom}\left(\pi_{1}(M), \pi_{0}(G)\right),
$$

e procedendo como no caso anterior, chegamos às classes livres:

$$
\left[M, B\left(G / G_{0}\right)\right]=\frac{\operatorname{Hom}\left(\pi_{1}(M), \pi_{0}(G)\right)}{P \pi_{0}(G)},
$$

onde novamente a ação é dada por $(\phi, \bar{\alpha})(x) \mapsto \phi^{\bar{\alpha}}(x)=\bar{\alpha}^{-1} \phi(x) \bar{\alpha}$, com $\bar{\alpha} \in$ $P \pi_{0}(G)=\operatorname{Inn}\left(\pi_{0} G\right)$.

Novamente pela proposição 26 , vemos que toda aplicação $f: X \rightarrow(B G)_{1}$ se levanta até $(B G)_{n}$ para qualquer $n$ - basta considerar a composta com a seção $B s: B\left(G / G_{0}\right) \rightarrow B G$ e a aplicação $p_{n}: B G \rightarrow(B G)_{n}$ compatível. Desta forma, precisamos apenas encontrar quantos levantamentos diferentes podemos ter, ou seja, precisamos determinar $\left(B p_{0}\right)_{*}^{-1}\left(B p_{0}\right)_{*}([f]) \operatorname{com}[f] \in[M, B G]_{0}$. Como $\left(B p_{0}\right)_{*}$ é sobrejetora, basta calcular $\left(B p_{0}\right)_{*}^{-1}([u])$ para todo $[u] \in\left[M, B\left(G / G_{0}\right)\right]_{0}$ e pelo que vimos acima, podemos identificar um elemento $[u] \in\left[M, B\left(G / G_{0}\right)\right]_{0}$ com seu correspondente $\phi_{u} \in \operatorname{Hom}\left(\pi_{1}(M), \pi_{0}(G)\right)$ tal que $\phi_{u}(1)=u_{\phi}$.

Assim, utilizando a seqüência espectral de Larmore (que nestas condições tem diferencial nula) temos que

$$
\left(B p_{0}\right)_{*}^{-1}\left(u_{\phi}\right)=H^{2}\left(M ; \pi_{1}\left(G, u_{\phi}\right)\right),
$$

onde, fixado $u_{\phi}$, os elementos de $\left(B p_{0}\right)_{*}^{-1}\left(u_{\phi}\right)$ são dados por $u_{\phi}+b$, onde $b \in$ $H^{2}\left(M ; \pi_{1}\left(G, u_{\phi}\right)\right)$ (notação estabelecida na observação 3.4). Assim,

$$
[M, B G]_{0}=\bigsqcup_{\phi \in H o m\left(\pi_{1}(M), \pi_{0}(G)\right)} H^{2}\left(M ; \pi_{1}\left(G, u_{\phi}\right)\right) .
$$


Para obtermos as classes livres, precisamos do quociente de $[M, B G]_{0}$ pela ação de $\pi_{0}(G)$. Utilizando o teorema 3.4, temos que

$$
\left(u_{\phi}+b\right)^{\alpha}=u_{\phi}^{\alpha}+1_{*}^{\alpha}(b)
$$

$\operatorname{com~} 1_{*}^{\alpha} \in \operatorname{Aut}\left(H^{2}\left(M ; \pi_{1}\left(G, u_{\phi}\right)\right)\right)$. Agora, $f \sim g$ em $[M, B G]_{0}$ se e somente se $u_{\phi}+a \sim v_{\psi}+b$ (aqui o sinal de igualdade significa equivalência de homotopia com ponto base, enquanto $\sim$ significa homotopia livre). Mas $u+a \sim v+b$ se e só se existe $\alpha \in \pi_{0}(G)$ tal que $(u+a)^{\alpha}=u^{\alpha}+1_{*}^{\alpha}(a)=v+b$. Como $1_{*}^{\alpha}$ é isomorfismo do grupo local de coeficientes, podemos identificar a menos de isomorfismo $1_{*}^{\alpha}(a) \operatorname{com} b$ e portanto $u+a \sim v+b \Longleftrightarrow v=u^{\alpha}$ para algum $\alpha \in \pi_{0}(G)$, uma vez identificados $1_{*}^{\alpha}(a)$ e $b$ pelo automorfismo $\alpha \in \pi_{0}(G) \subset A u t\left(H^{2}\left(M, \pi_{1}\left(G, u_{\phi}\right)\right)\right)$.

Segue daí que para $\operatorname{dim}(M)=2$ ou 3 , temos:

$$
[M, B G]=\bigsqcup_{\bar{\phi} \in \frac{\operatorname{Hom}\left(\pi_{1}(M), \pi_{0}(G)\right)}{P \pi_{0}(G)}} \frac{H^{2}\left(M ; \pi_{1}\left(G, u_{\phi}\right)\right)}{\pi_{0}(G)},
$$

Corolário 3. Assuma que o grupo $\pi_{0}(G)$ é abeliano (em particular se $G$ é abeliano) e então

$$
\begin{gathered}
\mathcal{B}\left(M^{1}, G\right)=H^{1}\left(M, \pi_{0}(G)\right) \\
\mathcal{B}\left(M^{2,3}, G\right)=\bigsqcup_{\phi \in \operatorname{Hom}\left(\pi_{1}(M), \pi_{0}(G)\right)} H^{2}\left(M ; \pi_{1}\left(G, u_{\phi}\right)\right) .
\end{gathered}
$$

Observação 4.1. Note que no caso conexo (teorema 4.1) se usarmos o teorema da estrutura para grupos de Lie conexos compactos, $\pi_{1}(G)$ é um grupo abeliano finitamente gerado, e então aplicando o teorema dos coeficientes universais podemos dizer que

$$
\mathcal{B}\left(M^{2,3}, G\right)=H^{2}(M ; \mathbb{Z}) \otimes \pi_{1}(G) \oplus H^{3}(M ; \mathbb{Z}) * \pi_{1}(G) .
$$

onde * denota o produto de torção. (Veja [7]).

Ainda, no caso compacto (teorema 4.2) se $M$ tem dimensão um e $\pi_{0}(G)$ é abeliano (em particular sempre que $G$ é abeliano), a ação por conjugação é trivial e daí

$\operatorname{Hom}\left(\pi_{1}(M), \pi_{0}(G)\right)=\operatorname{Hom}\left(H_{1}(M), \pi_{0}(G)\right)=H^{1}(M) \otimes \pi_{0}(G) \oplus H^{2}(M) * \pi_{0}(G)$, onde * denota o produto de torção novamente. 


\section{Capítulo 5}

\section{Fibrados sobre spherical space forms tridimensionais.}

Neste capítulo nós enumeramos completamente os elementos do conjunto das classes de equivalência de fibrados vetoriais de dimensões 2 e 3 sobre as spherical space forms. Os principais resultados podem ser encontrados de maneira resumida nas tabelas ao final do capítulo.

\subsubsection{Spherical space forms}

Esta seção tem o objetivo de fazer uma breve introdução ao tema das spherical space forms e fixar a notação para a parte final do trabalho. A principal referência aqui são os trabalhos de J. A. Wolf em [26]. A definição a seguir é necessária para sabermos do que se trata uma spherical space form:

Definição 5.1. Seja $M$ uma variedade diferenciável $n$-dimensional com uma conecção $H$ no seu frame bundle. Então $M$ é dita completa se toda geodésica maximal de $M$ é completa, ou seja, da forma $\alpha(t),-\infty \leq t \leq \infty$

O teorema a seguir pode ser encontrado em [26]:

Teorema 5.1 (W. Killing, H. Hopf). Seja $M^{n}$ uma variedade riemanniana de dimensão maior ou igual a dois e seja $k$ um número real. Então $M^{n}$ é completa, conexa e de curvatura constante $k$ se e somente se é isométrica a um quociente do tipo:

- $S^{n} / \Gamma \operatorname{com} \Gamma \subset O(n+1)$, se $K>0$; 
- $\mathbb{R}^{n} / \Gamma \operatorname{com} \Gamma \subset E(n)$, se $K=0$;

- $\mathbb{H}^{n} / \Gamma \operatorname{com} \Gamma \subset O^{1}(n+1)$, se $K<0$,

onde $\Gamma$ age livremente e propriamente descontinuamente .

Se uma variedade riemanniana completa conexa tem curvatura constante $k<0$, ela é chamada de "hyperbolic space form", se tem curvatura constante $k=0$, é chamada de "eulidean space form" e para o caso restante temos a seguinte

Definição 5.2. Uma spherical space form é uma variedade riemanniana completa conexa de curvatura positiva constante.

Portanto, as spherical space forms podem ser vistas como quocientes $S_{\Gamma}=S^{n} / \Gamma$ de esferas por grupos descontínuos de isometrias que agem sem pontos fixos. É desta forma que pensaremos estes objetos neste trabalho, fixando $n=3$ e fazendo a spherical space form depender, desta forma, apenas do grupo $\Gamma$ que age em $S^{3}$. Justamente por isso, às vezes nos referimos à spherical space form citando apenas o grupo $G$ em questão.

A classificação das spherical space forms tridimensionais foi obtida nos trabalhos de Seifert e Therelfall [20], predominantemente a partir dos trabalhos de H. Hopf [8] e Zassenhaus [27]. A história desta classificação começa em 1873 quando W. K. Clifford encontrou alguns mergulhos isométricos de toros planos em espaços tridimensionais elípticos. Alguns anos depois F. Klein se interessou pelo assunto e propôs a questão de saber se é possível encontrar todas as superfícies tridimensionais de curvatura gaussiana constante - um problema que depois foi generalizado para dimensão $n$ e reduzido a um problema de variedades de recobrimento, recebendo o nome de "Clifford-Klein space form problem". É interessante notar que no início do século $\mathrm{XX}$ a pesquisa sobre este problema foi fortemente desencorajada devido aos estudos de P. Enriques, que foram mais tarde corrigidos pelos trabalhos do próprio Hopf. Isso abriu espaço para os trabalhos de Seifert e Therelfall, dos quais faremos uso. Tal classificação tem a particularidade de depender de fenômenos peculiares à dimensão três, porém, como pode ser visto em [26], é possível obter uma classificação para spherical space forms de dimensão arbitrária.

Exibimos agora a tal classificação, dada a partir dos grupos que agem sem pontos fixos na esfera, como explicado acima; maiores detalhes podem ser vistos em [20].

1. O grupo cíclico $C(n)=<x: x^{n}>$;

2. O grupo dos quatérnios generalizados $Q(4 n)=\left\langle x, y: x^{n}=y^{2}, x y x=y\right\rangle$; 
3. O grupo tetraédrico binário $T^{*}(24)=<x, y: y x y=x^{2}, x y x=y^{2}>$;

4. O grupo octaédrico binário $O^{*}(48)=<x, y: x y x=y x y, x y^{2} x=y^{2}>$;

5. O grupo icosaédrico binário $I^{*}(120)=<x, y: x y^{2} x=y x y, y x^{2} y=x y x>$;

6. O produto semi-direto $C(2 n+1) \rtimes C\left(2^{k}\right)=<x, y: x^{2^{k}}=y^{2 n+1}=1, x y x^{-1}=$ $y^{-1}>, k \geq 2, n \geq 1$

7. O produto semi-direto $Q(8) \rtimes C\left(3^{k}\right)=<x, y, z: x^{2}=(x y)^{2}=y^{2}, z^{3^{k}}=$ $1, z x z^{-1}=y, z y z^{-1}=x y>, k \geq 1$;

8. O produto de qualquer grupo acima com um grupo cíclico de ordem coprima.

Como $S_{\Gamma}$ é o 3-esqueleto do espaço de Eilemberg-MacLane $K(1, \Gamma)$, e todos os grupos acima são finitos e finitamente apresentados, o $\mathbb{Z} \Gamma$-complexo de cadeias para o espaço de recobrimento universal $\tilde{S}_{\Gamma}\left(=S^{3}\right)$ é dado pela resolução vista em 2.3 (detalhes em [1]):

Proposição 27. Um $\mathbb{Z} \Gamma$-complexo de cadeias para o recobrimento universal $\tilde{S}_{\Gamma} d a$ forma espacial esférica $S=S^{3} / \Gamma$ é dado por

$$
0 \longrightarrow C_{3} \underset{d_{3}}{\longrightarrow} C_{2} \underset{d_{2}}{\longrightarrow} C_{1} \underset{d_{1}}{\longrightarrow} C_{0} \longrightarrow 0
$$

com

$$
C_{0}=\mathbb{Z} \Gamma[e], \quad C_{1}=\mathbb{Z} \Gamma[B], \quad C_{2}=\mathbb{Z} \Gamma[A], \quad C_{3}=\mathbb{Z} \Gamma[C],
$$

onde $\Gamma$ tem apresentação $\Gamma=<S ; T>$, e as notações e os homomorfismos são como definidos na proposição 22.

Como não é trivial calcular explicitamente o kernel de $d_{2}$ para qualquer grupo, nós consideramos o complexo de cadeias apenas até o nível 2. De qualquer forma, podemos calcular os grupos de cohomolgia torcida a partir da seguinte generalização do teorema da dualidade de Poincaré, que é válida aqui sem restrições porque todas as variedades são orientáveis [4]:

Proposição 28. : Para uma variedade orientável $M$ de dimensão $m$, e um $\mathbb{Z} G$ módulo $A$ dado por qualquer representação $\rho: G \rightarrow A u t(A)$, o produto cap pela classe fundamental $[M]$ nos dá um isomorfismo

$$
\cap[M]: H^{k}\left(M ; A_{\rho}\right) \cong H_{m-k}\left(M ; A_{\rho}\right) .
$$




\subsubsection{Cálculos}

Vamos agora mostrar os cálculos necessários para aplicar o teorema 4.2 (de fato, é suficiente usar o corolário 3) e então obter os fibrados vetoriais reais sobre as spherical space forms tridimensionais. Se $\Gamma$ é um dos grupos listados na seção 5.0.1, denotaremos $M=S_{\Gamma}, G=O_{n}, G_{0}=S O_{n}, \quad G / G_{0}=\pi_{0}(G)=\mathbb{Z} / 2$ e $\pi_{2}(B G)=$ $\pi_{1}(G)=0, \mathbb{Z}$ ou $\mathbb{Z} / 2$ para $n=1,2,3$. Note que $G$ é abeliano e do fato de que $B O$ é simples (veja [11]), temos que $B G$ é 2 -simples em todos os casos, exceto quando $G=O_{2}$ e a ação de $\pi_{1}\left(B O_{2}\right)$ em $\pi_{2}\left(B O_{2}\right)$ corresponde à mudança de sinal (ou equivalentemente à mudança de orientação local do fibrado). Na realidade este caso nunca ocorrerá aqui, como é possível verificar nas tabelas finais.

A cada caso, vamos primeiro calcular $\operatorname{Hom}\left(\pi_{1}\left(S_{\Gamma}\right), \pi_{0}(G)\right) \approx \operatorname{Hom}(A b(\Gamma), \mathbb{Z} / 2)$, que pelo corolário 3 , corresponde ao conjunto dos fibrados reais por linha sobre $S_{\Gamma}$. Depois, para aplicar a seqüência espectral de Larmore para enumerar fibrados de dimensões mais altas, precisaremos da cohomologia de $S_{\Gamma}$ torcida por tais fibrados por linha.

Seja então $u$ um elemento em $\left[S_{\Gamma}, B\left(G / G_{0}\right)\right]$, que classifica os fibrados por linha, e seja $\phi_{u}$ em $\operatorname{Hom}\left(\pi_{1}\left(S_{\Gamma}\right), \pi_{0}(G)\right)$ o correspondente homomorfismo, como na prova de 4.2. Precisamos calcular $H^{2}\left(S_{\Gamma} ; \pi_{1}(G, u)\right)$. Quando $G=O_{2}$ por exemplo, $(B G)_{1}=$ $G / G_{0}$, o sheaf $\pi_{1}(G, u)$ com fibra $\pi_{1}(G) \approx \mathbb{Z}$ e grupo estrutural $\pi_{0}(G) \approx \mathbb{Z} / 2$, agindo pelo automorfismo determinado por uma representação $\rho: \pi_{0}(G) \rightarrow \operatorname{Aut}\left(\pi_{1}(G)\right)$, corresponde e é classificado pelos homomorfismos de torção $\phi: \pi_{1}\left(S_{\Gamma}\right) \rightarrow \pi_{0}(G)$, ou seja, podemos identificar

$$
H^{2}\left(S_{\Gamma} ; \pi_{1}\left(G, u_{\phi}\right)\right)=H^{2}\left(S_{\Gamma} ; \mathbb{Z}_{\rho_{\phi}}\right) .
$$

Nos outros casos, precisaremos apenas do sheaf trivial.

Desta forma, para cada $\Gamma$ e cada representação aceita por este, daremos a forma explícita do complexo de cadeias descrito na proposição 22 , e também calculamos os grupo de homologia torcida.

Fixaremos a seguinte notação para representação de grupos: para um subconjunto $W$ do conjunto dos geradores de $\Gamma$, seja $\rho_{W}: \Gamma \rightarrow A u t(\mathbb{Z})$ a notação para o homomorfimo $\rho_{W}(W)=-1, \quad \rho_{W}(S \backslash W)=1$. Denotaremos ainda por $\rho_{0}$ a representação trivial. Note que nem todo $W$ define um homomorfismo: as relações de uma representação $\Gamma$ impõe certas restrições aqui. Sempre que conhecemos um complexo de cadeias completo para algum dos grupos abaixo, nós o fornecemos explicitamente. 
Grupos cíclicos, $C(t)=<x, x^{t}>$

Este primeiro caso será mostrado aqui em maiores detalhes, para os demais casos exibiremos todas as ferramentas necessárias para proceder com os cálculos, porém estes não serão apresentados.

É conseqüência imediata da apresentação dos grupos cíclicos $C(t)$ que quando $t=2 n+1$ não existe um homomorfismo $\rho_{x}$ de $C(t)$ em $\mathbb{Z} / 2$ de maneira que o gerador $x$ seja levado no elemento não trivial $-1 \in \mathbb{Z} / 2$, uma vez que teríamos $1=\rho_{x}(1)=\rho_{x}\left(x^{t}\right)=-1 \in \mathbb{Z} / 2$.

Assim sendo, estas são as únicas representações aceitas:

$$
\begin{aligned}
& \operatorname{Hom}(C(2 n+1), \mathbb{Z} / 2)=\left\{\rho_{0}\right\}, \\
& \operatorname{Hom}(C(2 n), \mathbb{Z} / 2)=\left\{\rho_{0}, \rho_{x}\right\} .
\end{aligned}
$$

A seguinte resolução periódica completa foi retirada de [5] e [2]:

$$
\begin{gathered}
0 \longrightarrow \mathbb{Z} \Gamma[d] \underset{d_{3}}{0} \mathbb{Z} \Gamma[c] \underset{d_{2}}{\longrightarrow} \mathbb{Z} \Gamma[b] \underset{d_{1}}{\longrightarrow} \mathbb{Z} \Gamma[a] \longrightarrow 0, \\
d_{1}(b)=(x-1) a, \\
d_{2}(c)=\left(1+x+x^{2}+\ldots+x^{t-1}\right) b, \\
d_{3}(d)=(x-1) c .
\end{gathered}
$$

As homologias são calculadas a partir desta resolução como se segue, onde $\langle a\rangle$ significa gerado por $a$.

- (Nível zero)

$$
H_{0}=\frac{\langle a\rangle}{\operatorname{Im}\left(d_{1}\right)}=\frac{<a\rangle}{<(x-1) a>},
$$

de onde se considerarmos $\rho_{0}$ teremos

$$
H_{0}=\frac{<a>}{1}=\mathbb{Z}
$$

e se considerarmos $\rho_{x}$ teremos

$$
H_{0}=\frac{<a>}{<-2 a>}=\mathbb{Z} / 2 .
$$

- (Nível um)A seguir temos

$$
H_{1}=\frac{<\operatorname{Ker}\left(d_{1}\right)>}{<\operatorname{Im}\left(d_{2}\right)>},
$$


agora, para $\rho_{0}$ temos $\operatorname{Ker}\left(d_{1}\right)=<b>$ enquanto para $\rho_{x}$ temos um kernel nulo para $d_{1}$. A imagem de $d_{2}$ no primeiro caso é gerada por $\left(1+1+1^{2}+\ldots+1^{t-1}\right) b=$ $t b$, assim

$$
H_{1}=\frac{<b>}{<t b>}=\mathbb{Z} / t
$$

se tomarmos $\rho_{0}$ e $H_{1}=0$ se tomarmos $\rho_{x}$.

- (Nível dois)

$$
H_{2}=\frac{<\operatorname{Ker}\left(d_{2}\right)>}{<\operatorname{Im}\left(d_{3}\right)>},
$$

agora, para $\rho_{0}$ o kernel é nulo e para $\rho_{x}$ temos $\operatorname{Ker}\left(d_{1}\right)=<c>$ e uma vez que a imagem de $d_{3}$ para $\rho_{x}$ é dada por $\langle(x-1) c\rangle=\langle 2 c\rangle$, temos $H_{2}=0$ para $\rho_{0}$ e $H_{2}=\mathbb{Z} / 2$ para $\rho_{x}$.

- (Nível três) De maneira completamente análoga aos casos anteriores, temos que para $\rho_{x}$ este grupo de homologia é nulo enquanto para $\rho_{0}$ é $\mathbb{Z}$.

A tabela a seguir reúne estes grupos de homologia:

\begin{tabular}{|c|c|c|c|c|}
\hline & $H_{0}$ & $H_{1}$ & $H_{2}$ & $H_{3}$ \\
\hline$\rho_{0}$ & $\mathbb{Z}$ & $\mathbb{Z} / t$ & 0 & $\mathbb{Z}$ \\
\hline$\rho_{x}$ & $\mathbb{Z} / 2$ & 0 & $\mathbb{Z} / 2$ & 0 \\
\hline
\end{tabular}

Ao dualizar estes resultados temos a cohomologia completa com coeficientes em $\mathbb{Z}$ para os grupos cíclicos. A partir desta e do teorema dos coeficientes universais para cohomologia temos a cohomologia com coeficientes globais em $\mathbb{Z}_{2}$ :

\begin{tabular}{|c|c|c|c|}
\hline$H^{0}$ & $H^{1}$ & $H^{2}$ & $H^{3}$ \\
\hline $\mathbb{Z} / 2$ & $\mathbb{Z} / m$ & $\mathbb{Z} / m$ & $\mathbb{Z} / 2$ \\
\hline
\end{tabular}

onde $m=\operatorname{gcd}(t, 2)$

Grupos quaterniônicos generalizados, $Q(4 n)=\left\langle x, y: x^{n}=y^{2}, x y x=y\right\rangle$

Representações aceitas

$$
\begin{gathered}
\operatorname{Hom}(Q(8 n), \mathbb{Z} / 2)=\left\{\rho_{0}, \rho_{x}, \rho_{y}, \rho_{x, y}\right\}, \\
\operatorname{Hom}(Q(8 n+4), \mathbb{Z} / 2)=\left\{\rho_{0}, \rho_{y}\right\} .
\end{gathered}
$$

Complexo de cadeias (note que neste caso sabemos também $C_{3}=\mathbb{Z} \Gamma[c][2]$ ) 


$$
\begin{gathered}
0 \longrightarrow \mathbb{Z} \Gamma[d] \underset{d_{3}}{\longrightarrow} \mathbb{Z} \Gamma\left[c_{1}, c_{2}\right] \underset{d_{2}}{\longrightarrow} \mathbb{Z} \Gamma\left[b_{1}, b_{2}\right] \underset{d_{1}}{\longrightarrow} \mathbb{Z} \Gamma[a] \longrightarrow 0, \\
d_{1}\left(b_{1}\right)=(x-1) a, \quad d_{1}\left(b_{2}\right)=(y-1) a, \\
d_{2}\left(c_{1}\right)=\Omega b_{1}+(-y-1) b_{2}, \quad d_{2}\left(c_{2}\right)=(x y+1) b_{1}+(x-1) b_{2}, \\
d_{3}(d)=(x-1) c_{1}+(-x y+1) c_{2},
\end{gathered}
$$

onde $\Omega=1+x+x^{2}+\ldots+x^{t-1}, t=2 n$ (caso de $Q(8 n)$ ) or $t=2 n+1$ (caso de $Q(8 n+4))$. Os grupos de homologia são:

\begin{tabular}{|c|c|c|c|c|}
\hline & $H_{0}$ & $H_{1}$ & $H_{2}$ & $H_{3}$ \\
\hline$\rho_{0}$ & $\mathbb{Z}$ & $\mathbb{Z} / 2 \oplus \mathbb{Z} / 2$ & 0 & $\mathbb{Z}$ \\
\hline$\rho_{x}$ & $\mathbb{Z} / 2$ & $\mathbb{Z} / 2$ & $\mathbb{Z} / 2$ & 0 \\
\hline$\rho_{y}$ & $\mathbb{Z} / 2$ & $\mathbb{Z} / t$ & $\mathbb{Z} / 2$ & 0 \\
\hline$\rho_{x, y}$ & $\mathbb{Z} / 2$ & $\mathbb{Z} / 2$ & $\mathbb{Z} / 2$ & 0 \\
\hline
\end{tabular}

e a $\mathbb{Z} / 2$-cohomologia é

\begin{tabular}{|c|c|c|c|}
\hline$H^{0}$ & $H^{1}$ & $H^{2}$ & $H^{3}$ \\
\hline 0 & $\mathbb{Z} / 2 \oplus \mathbb{Z} / 2$ & $\mathbb{Z} / 2 \oplus \mathbb{Z} / 2$ & $\mathbb{Z} / 2$ \\
\hline
\end{tabular}

Nos próximos três casos a resolução foi tirada de [23].

O grupo tetraédrico binário, $T^{*}(24)=<x, y: y x y=x^{2}, x y x=y^{2}>$

$$
\begin{gathered}
\operatorname{Hom}\left(T^{*}(24), \mathbb{Z} / 2\right)=\left\{\rho_{0}\right\}, \\
\cdots \longrightarrow \mathbb{Z} \Gamma[d] \underset{d_{3}}{\longrightarrow} \mathbb{Z} \Gamma\left[c_{1}, c_{2}\right] \underset{d_{2}}{\longrightarrow} \mathbb{Z} \Gamma\left[b_{1}, b_{2}\right] \underset{d_{1}}{\longrightarrow} \mathbb{Z} \Gamma[a] \longrightarrow 0, \\
d_{1}\left(b_{1}\right)=(x-1) a, \quad d_{1}\left(b_{2}\right)=(y-1) a, \\
d_{2}\left(c_{1}\right)=\left(y-y x y s^{-1}-y x y x^{-2}\right) b_{1}+(1+y x) b_{2}, \\
d_{2}\left(c_{2}\right)=(1+x y) b_{1}+\left(x-x y x y^{-1}-x y x y^{-2}\right) b_{2} . \\
d_{3}(d)=(x-1) c_{1}+(y-1) c_{2}
\end{gathered}
$$

\begin{tabular}{|c|c|c|c|c|}
\hline & $H_{0}$ & $H_{1}$ & $H_{2}$ & $H_{3}$ \\
\hline$\rho_{0}$ & $\mathbb{Z}$ & $\mathbb{Z}_{3}$ & 0 & $\mathbb{Z}$ \\
\hline
\end{tabular}


$\mathbb{Z} / 2$-cohomologia:

\begin{tabular}{|c|c|c|c|}
\hline$H^{0}$ & $H^{1}$ & $H^{2}$ & $H^{3}$ \\
\hline $\mathbb{Z} / 2$ & 0 & 0 & $\mathbb{Z} / 2$ \\
\hline
\end{tabular}

O grupo octaédrico binário, $O^{*}(48)=<x, y: x y x=y x y, x y^{2} x=y^{2}>$

$$
\begin{gathered}
\operatorname{Hom}\left(O^{*}(48), \mathbb{Z} / 2\right)=\left\{\rho_{0}, \rho_{x, y}\right\}, \\
\cdots \longrightarrow \mathbb{Z} \Gamma[d] \underset{d_{3}}{\longrightarrow} \mathbb{Z} \Gamma\left[c_{1}, c_{2}\right] \underset{d_{2}}{\longrightarrow} \mathbb{Z} \Gamma\left[b_{1}, b_{2}\right] \underset{d_{1}}{\longrightarrow} \mathbb{Z} \Gamma[a] \longrightarrow 0, \\
d_{1}\left(b_{1}\right)=(x-1) a, \quad d_{1}\left(b_{2}\right)=(y-1) a, \\
d_{2}\left(c_{1}\right)=(1-y+x y) b_{1}+(x-1-y x) b_{2}, \quad d_{2}\left(c_{2}\right)=\left(1+x y^{2}\right) b_{1}+(x-1-y+x y) b_{2} . \\
d_{3}(d)=(1-x y) c_{1}+(y-1) c_{2}
\end{gathered}
$$

\begin{tabular}{|c|c|c|c|c|}
\hline & $H_{0}$ & $H_{1}$ & $H_{2}$ & $H_{3}$ \\
\hline$\rho_{0}$ & $\mathbb{Z}$ & $\mathbb{Z} / 2$ & 0 & $\mathbb{Z}$ \\
\hline$\rho_{x, y}$ & $\mathbb{Z} / 2$ & $\mathbb{Z} / 3$ & $\mathbb{Z} / 2$ & 0 \\
\hline
\end{tabular}

$\mathbb{Z} /$-cohomologia:

\begin{tabular}{|c|c|c|c|}
\hline$H^{0}$ & $H^{1}$ & $H^{2}$ & $H^{3}$ \\
\hline $\mathbb{Z} / 2$ & $\mathbb{Z} / 2$ & $\mathbb{Z} / 2$ & $\mathbb{Z} / 2$ \\
\hline
\end{tabular}

O grupo icosaédrico binário, $I^{*}(120)=<x, y: x y^{2} x=y x y, y x^{2} y=x y x>$

$$
\begin{gathered}
\operatorname{Hom}\left(I^{*}(120), \mathbb{Z} / 2\right)=\left\{\rho_{0}\right\}, \\
\cdots \longrightarrow \mathbb{Z} \Gamma[d] \underset{d_{3}}{\longrightarrow} \mathbb{Z} \Gamma\left[c_{1}, c_{2}\right] \underset{d_{2}}{\longrightarrow} \mathbb{Z} \Gamma\left[b_{1}, b_{2}\right] \underset{d_{1}}{\longrightarrow} \mathbb{Z} \Gamma[a] \longrightarrow 0, \\
d_{1}\left(b_{1}\right)=(x-1) a, \quad d_{1}\left(b_{2}\right)=(y-1) a, \\
d_{2}\left(c_{1}\right)=\left(1+x y^{2}-x y^{2} x y^{-1} x^{-1}\right) b_{1}+\left(x+x y-x y^{2} x y^{-1}-x y^{2} x y^{-1} x^{-1} y^{-1}\right) b_{2}, \\
d_{2}\left(c_{2}\right)=\left(y+y x-y x^{2} y x^{-1}-y x^{2} y x^{-1} y^{-1} x^{-1}\right) b_{1}+(x-1-y+x y) b_{2} . \\
d_{3}(d)=(1-y x) c_{1}+(1-x y) c_{2}
\end{gathered}
$$




\begin{tabular}{|c|c|c|c|c|}
\hline & $H_{0}$ & $H_{1}$ & $H_{2}$ & $H_{3}$ \\
\hline$\rho_{0}$ & $\mathbb{Z}$ & 0 & 0 & $\mathbb{Z}$ \\
\hline
\end{tabular}

$\mathbb{Z} / 2$-cohomologia:

\begin{tabular}{|c|c|c|c|}
\hline$H^{0}$ & $H^{1}$ & $H^{2}$ & $H^{3}$ \\
\hline $\mathbb{Z} / 2$ & 0 & 0 & $\mathbb{Z} / 2$ \\
\hline
\end{tabular}

O grupo $\mathbf{C}(2 n+1) \rtimes C\left(2^{k}\right)=<x, y: x^{2^{k}}=y^{2 n+1}=1, x y x^{-1}=y^{-1}>, k \geq 2$, $n \geq 1$.

$$
\begin{gathered}
\operatorname{Hom}\left(C(2 n+1) \rtimes C\left(2^{k}\right), \mathbb{Z} / 2\right)=\left\{\rho_{0}, \rho_{x}\right\} \\
\cdots \longrightarrow \mathbb{Z} \Gamma\left[c_{1}, c_{2}, c_{3}\right] \underset{d_{2}}{\longrightarrow} \mathbb{Z} \Gamma\left[b_{1}, b_{2}\right] \underset{d_{1}}{\longrightarrow} \mathbb{Z} \Gamma[a] \longrightarrow 0 \\
d_{1}\left(b_{1}\right)=(x-1) a, \quad d_{1}\left(b_{2}\right)=(y-1) a \\
d_{2}\left(c_{1}\right)=\left(1+x+\ldots+x^{2^{k}-1}\right) b_{1}+(0) b_{2}, \\
d_{2}\left(c_{2}\right)=(0) b_{1}+\left(1+y+\ldots+y^{2 n}\right) b_{2}, \\
d_{2}\left(c_{3}\right)=\left(1-y^{-1}\right) b_{1}+\left(x+y^{-1}\right) b_{2} .
\end{gathered}
$$

\begin{tabular}{|c|c|c|}
\hline & $H_{0}$ & $H_{1}$ \\
\hline$\rho_{0}$ & $\mathbb{Z}$ & $\mathbb{Z} / 2^{k}$ \\
\hline$\rho_{x}$ & $\mathbb{Z} / 2$ & 0 \\
\hline
\end{tabular}

$\mathbb{Z} / 2$-cohomologia:

\begin{tabular}{|c|c|c|}
\hline$H^{1}$ & $H^{2}$ & $H^{3}$ \\
\hline $\mathbb{Z}_{2}$ & $\mathbb{Z} / 2 \oplus \mathbb{Z} / 2$ & $\mathbb{Z} / 2$ \\
\hline
\end{tabular}

O grupo $Q(8) \rtimes C\left(3^{k}\right)=<x, y, z: x^{2}=(x y)^{2}=y^{2}, z^{3^{k}}=1, z x z^{-1}=y, z y z^{-1}=$ $x y>, k \geq 1$.

$$
\operatorname{Hom}\left(Q(8) \rtimes C\left(3^{k}\right), \mathbb{Z} / 2\right)=\left\{\rho_{0}\right\},
$$




$$
\begin{aligned}
& \cdots \longrightarrow \mathbb{Z} \Gamma\left[c_{1}, c_{2}, c_{3}, c_{4}, c_{5}, c_{6}\right] \underset{d_{2}}{\longrightarrow} \mathbb{Z} \Gamma\left[b_{1}, b_{2}, b_{3}\right] \underset{d_{1}}{\longrightarrow} \mathbb{Z} \Gamma[a] \longrightarrow 0, \\
& d_{1}\left(b_{1}\right)=(x-1) a, \quad d_{1}\left(b_{2}\right)=(y-1) a \quad d_{1}\left(b_{3}\right)=(z-1) a, \\
& d_{2}\left(c_{1}\right)=\left(1+x y-x y x y x^{-1}-x y x y x^{-2}\right) b_{1}+(x+x y x) b_{2}+(0) b_{3} \text {, } \\
& d_{2}\left(c_{2}\right)=(1+x y) b_{1}+\left(x-x y x y^{-1}\right) b_{2}+(0) b_{3}, \\
& d_{2}\left(c_{3}\right)=(1+x) b_{1}+\left(x^{2}+x^{2} y\right) b_{2}+(0) b_{3}, \\
& d_{2}\left(c_{4}\right)=(0) b_{1}+(0) b_{2}+\left(1+z+\ldots+z^{3^{k}-1}\right) b_{3}, \\
& d_{2}\left(c_{5}\right)=(z) b_{1}+\left(-z x z^{-1} y^{-1}\right) b_{2}+\left(1-z x z^{-1}\right) b_{3}, \\
& d_{2}\left(c_{6}\right)=\left(-z y z^{-1} y^{-1} x^{-1}\right) b_{1}+\left(z-z y z^{-1} y^{-1}\right) b_{2}+\left(1-z y z^{-1}\right) b_{3} . \\
& \begin{array}{|c|c|c|}
\hline & H_{0} & H_{1} \\
\hline \rho_{0} & \mathbb{Z} & \mathbb{Z} / 3^{k} \\
\hline
\end{array}
\end{aligned}
$$

$\mathbb{Z} / 2$-cohomologia:

\begin{tabular}{|c|c|c|}
\hline$H^{1}$ & $H^{2}$ & $H^{3}$ \\
\hline 0 & 0 & $\mathbb{Z} / 2$ \\
\hline
\end{tabular}

\subsection{Enumerando fibrados}

Nas tabelas a seguir classificamos todos os fibrados vetoriais reais de dimensão menor ou igual a três sobre as spherical space forms de dimensão três. A notação é estabalecida da seguinte maneira: Na primeira tabela, para cada grupo $\Gamma$, a primeira coluna exibe os fibrados por linha enumerados por suas classes de Stiefel-Whitney; assim, 1 denota o fibrado trivial. Os elementos $\alpha_{s}$ são geradores fixos de $H^{1}\left(S_{\Gamma} ; \mathbb{Z} / 2\right)$ - conjunto este que pode ser identificado com $\operatorname{Hom}(\Gamma, \mathbb{Z} / 2)$. Na segunda coluna estão classificados, para cada fibrado por linha com primeira classe de Stiefel-Whitney $\alpha_{s}$, os 2-fibrados com a mesma classe de Stiefel-Whitney. Tais 2-fibrados são enumerados por expressões como $1+\alpha_{s}+y$, onde y é a classe de obstrução em $H^{2}\left(S_{\Gamma} ; \mathbb{Z}_{\rho_{s}}\right)$. Ainda, os $\beta_{i}$ são geradores fixos de $H^{2}\left(S_{\Gamma} ; \mathbb{Z}_{\rho_{s}}\right)$. Na segunda tabela estão os 3 fibrados vetoriais com a mesma notação (salvo que os $\beta_{i}$ neste caso são geradores de $\left.H^{2}\left(S_{\Gamma} ; \mathbb{Z} / 2\right)\right)$. 


\begin{tabular}{|c|c|c|c|}
\hline \multicolumn{2}{|l|}{$\Gamma$} & $V e c t_{1}\left(S_{\Gamma}\right)$ & $V \operatorname{ect}_{2}\left(S_{\Gamma}\right)$ \\
\hline \multirow{3}{*}{$\mathrm{C}(\mathrm{t})$} & \multirow{2}{*}{$\mathrm{t}=2 \mathrm{n}$} & 1 & $1+m \beta, 0 \leq m<t$ \\
\hline & & $1+\alpha_{x}$ & $1+\alpha_{x}$ \\
\hline & $t=2 n+1$ & 1 & $1+m \beta, 0 \leq m<t$ \\
\hline \multirow{6}{*}{$\mathrm{Q}(4 \mathrm{t})$} & \multirow{4}{*}{$\mathrm{t}=2 \mathrm{n}$} & 1 & $1,1+\beta_{1}, 1+\beta_{2}, 1+\beta_{1}+\beta_{2}$ \\
\hline & & $1+\alpha_{x}$ & $1+\alpha_{x}, 1+\alpha_{x}+\beta$ \\
\hline & & $1+\alpha_{y}$ & $1+\alpha_{y}+m \beta, 0 \leq m<t$ \\
\hline & & $1+\alpha_{x}+\alpha_{y}$ & $1+\alpha_{x}+\alpha_{y}, 1+\alpha_{x}+\alpha_{y}+\beta$ \\
\hline & \multirow{2}{*}{$\mathrm{t}=2 \mathrm{n}+1$} & 1 & $1,1+\beta_{1}, 1+\beta_{2}, 1+\beta_{1}+\beta_{2}$ \\
\hline & & $1+\alpha_{y}$ & $1+\alpha_{y}+m \beta, 0 \leq m<t$ \\
\hline$T^{*}(24)$ & & 1 & $1+m \beta, 0 \leq m<3$ \\
\hline \multirow{2}{*}{$O^{*}(48)$} & & 1 & $1,1+\beta$ \\
\hline & & $1+\alpha_{x}+\alpha_{y}$ & $1+\alpha_{x}+\alpha_{y}+m \beta, 0 \leq m<3$ \\
\hline$I^{*}(120)$ & & 1 & 1 \\
\hline \multirow{2}{*}{\multicolumn{2}{|c|}{$C(2 n+1) \rtimes C\left(2^{k}\right)$}} & 1 & $1+m \beta, 0 \leq m<2^{k}$ \\
\hline & & $1+\alpha_{x}$ & $1+\alpha_{x}+m \beta, 0 \leq m<2 n+1$ \\
\hline$Q(8) \rtimes C\left(3^{k}\right)$ & & 1 & $1+m \beta, 0 \leq m<3^{k}$ \\
\hline
\end{tabular}




\begin{tabular}{|c|c|c|c|}
\hline \multicolumn{2}{|l|}{$\Gamma$} & $\operatorname{Vect}_{1}\left(S_{\Gamma}\right)$ & $\operatorname{Vect}_{3}\left(S_{\Gamma}\right)$ \\
\hline \multirow{3}{*}{$\mathrm{C}(\mathrm{t})$} & \multirow{2}{*}{$\mathrm{t}=2 \mathrm{n}$} & 1 & $1,1+\beta$ \\
\hline & & $1+\alpha_{x}$ & $1+\alpha_{x}, 1+\alpha_{x}+\beta$ \\
\hline & $t=2 n+1$ & 1 & 1 \\
\hline \multirow{6}{*}{$\mathrm{Q}(4 \mathrm{t})$} & \multirow{4}{*}{$\mathrm{t}=2 \mathrm{n}$} & 1 & $1,1+\beta_{1}, 1+\beta_{2}, 1+\beta_{1}+\beta_{2}$ \\
\hline & & $1+\alpha_{x}$ & $1+\alpha_{x}, 1+\alpha_{x}+\beta_{1}, 1+\alpha_{x}+\beta_{2}, 1+\alpha_{x}+\beta_{1}+\beta_{2}$ \\
\hline & & $1+\alpha_{y}$ & $1+\alpha_{y}, 1+\alpha_{y}+\beta_{1}, 1+\alpha_{y}+\beta_{2}, 1+\alpha_{y}+\beta_{1}+\beta_{2}$ \\
\hline & & $1+\alpha_{x}+\alpha_{y}$ & $\begin{array}{c}1+\alpha_{x}+\alpha_{y}, 1+\alpha_{x}+\alpha_{y}+\beta_{1} \\
1+\alpha_{x}+\alpha_{y}+\beta_{2}, 1+\alpha_{x}+\alpha_{y}+\beta_{1}+\beta_{2}\end{array}$ \\
\hline & \multirow{2}{*}{$\mathrm{t}=2 \mathrm{n}+1$} & 1 & $1,1+\beta_{1}, 1+\beta_{2}, 1+\beta_{1}+\beta_{2}$ \\
\hline & & $1+\alpha_{y}$ & $1+\alpha_{y}, 1+\alpha_{y}+\beta_{1}, 1+\alpha_{y}+\beta_{2}, 1+\alpha_{y}+\beta_{1}+\beta_{2}$ \\
\hline$T^{*}(24)$ & & 1 & 1 \\
\hline \multirow{2}{*}{$O^{*}(48)$} & & 1 & $1,1+\beta$ \\
\hline & & $1+\alpha_{x}+\alpha_{y}$ & $1+\alpha_{x}+\alpha_{y}, 1+\alpha_{x}+\alpha_{y}+\beta$ \\
\hline$I^{*}(120)$ & & 1 & 1 \\
\hline \multirow{2}{*}{\multicolumn{2}{|c|}{$C(2 n+1) \rtimes C\left(2^{k}\right)$}} & 1 & $1,1+\beta_{1}, 1+\beta_{2}, 1+\beta_{1}+\beta_{2}$ \\
\hline & & $1+\alpha_{x}$ & $1+\alpha_{x}, 1+\alpha_{x}+\beta_{1}, 1+\alpha_{x}+\beta_{2}, 1+\alpha_{x}+\beta_{1}+\beta_{2}$ \\
\hline$Q(8) \rtimes C\left(3^{k}\right)$ & & 1 & 1 \\
\hline
\end{tabular}




\section{Capítulo 6}

\section{Apêndice}

\subsection{O produto Cup}

O produto cup é essencial sempre que lidamos com anéis de cohomologia por fornecer a estrutura multiplicativa para estes anéis. Aqui vamos descrever rapidamente o que é o produto cup e calcular a estrutura multiplicativa de determinados anéis de cohomologia.

Se tomarmos como grupo a soma direta $\bigoplus_{i}\left[H^{i}(X)\right]$ então é possível definir um tipo de produto, chamado produto cup, de tal forma que este grupo se torne um anel, o anel de cohomologia do espaço $X$.

Para definirmos tal ferramenta, vamos considerar a cohomologia com coeficientes num anel $R$ qualquer. O que faremos aqui é definir um produto cup para cocadeias que induz um produto cup para classes de homologia. A prova desta indução e demais pormenores sobre o produto cup podem ser encontrados em [6].

Definição 6.1. Dada uma $r$ - cocadeia $c^{r} \in C^{r}(X, R)$ e uma $s$ - cocadeia $c^{s} \in$ $C^{s}(X, R)$, definimos o produto cup entre $c^{r}$ e $c^{s}$ como sendo a $(r+s)$ - cocadeia $c^{r} \smile c^{s} \in C^{r+s}(X, R)$ dada por

$$
c^{r} \smile c^{s}(\sigma)=c^{r}\left(\sigma \mid\left[v_{0}, \ldots, v_{r}\right]\right) c^{s}\left(\sigma \mid\left[v_{r}, \ldots, v_{r+s}\right]\right),
$$

onde o produto efetuado no termo da direita é o produto do próprio anel $R$.

\subsubsection{A estrutura multiplicativa do anel $H^{*}(Q(2 n) ; R)$.}

Nosso objetivo aqui será explicitar a estrutura multiplicativa do anel $H^{*}(Q(2 n) ; R)$, calculando o produto cup para a cohomologia de $Q(2 n)$ com coeficientes globais em 
$\mathbb{Z} / 2, \mathbb{Z}$ e para todos os coeficientes globais vistos na seção 2.2. Os resultados podem ser conferidos nas tabelas ao longo desta seção.

De acordo com a subseção 5.0.2, temos um complexo de cadeias $(\mathbb{Z} Q(2 n)$-resolução livre sobre $\mathbb{Z}$ ):

$$
X=0 \longrightarrow \mathbb{Z} \Gamma[d] \underset{d_{3}}{\longrightarrow} \mathbb{Z} \Gamma\left[c_{1}, c_{2}\right] \underset{d_{2}}{\longrightarrow} \mathbb{Z} \Gamma\left[b_{1}, b_{2}\right] \underset{d_{1}}{\longrightarrow} \mathbb{Z} \Gamma[a] \longrightarrow 0,
$$

e para alcançar nosso objetivo, precisamos explicitar uma aplicação de cadeias $\varphi$ : $X \rightarrow X \otimes X$ tal que:

a) $\varphi$ é uma $Q(2 n)$-aplicação que age diagonalmente em $X \otimes X$;

b) O diagrama abaixo comuta:

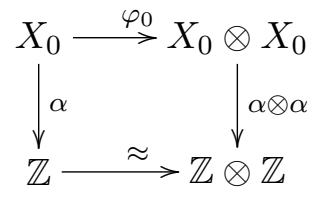

Então, tomando $Q(2 n)=<x, y: x^{n}=y^{2}, \quad x y x^{-1}=y>$, usaremos a mesma aplicação $\varphi$ encontrada em [22], dada explicitamente por:

$$
\begin{aligned}
& \varphi(a)=a \otimes a \\
& \varphi(b)=b \otimes x a+a \otimes b \quad \varphi\left(b^{\prime}\right)=b^{\prime} \otimes y a+a \otimes b^{\prime} \\
& \varphi(c)=a \otimes c+c \otimes y^{2} a+\sum_{0 \leq i \leq j<n} x^{i} b \otimes x^{j} b-b^{\prime} \otimes y b^{\prime} \quad \varphi\left(c^{\prime}\right)=a \otimes c^{\prime}+b \otimes x b^{\prime}+b^{\prime} \otimes x y b-x y b \otimes x y b+c^{\prime} \otimes x y a \\
& \varphi(e)=a \otimes e+b \otimes x c+x c \otimes y^{2} b-c^{\prime} \otimes\left(x y b+b^{\prime}\right)-\left(b+x b^{\prime}\right) \otimes r x y c^{\prime}+e \otimes y^{2} a
\end{aligned}
$$

Vamos encarar o produto cup como sendo a seguinte composta:

$$
\begin{gathered}
H^{i}(X ; R) \times H^{j}(X ; R) \stackrel{\otimes}{\longrightarrow} H^{i+j}(X \otimes X) \stackrel{\varphi}{\longrightarrow} H^{i+j}(X ; R) \\
h^{1} \times h^{2} \longmapsto h^{1} \otimes h^{2} \longmapsto h^{*}=h^{1} \smile h^{2}
\end{gathered}
$$

e portanto para uma $(i+j)$-cadeia $c$ qualquer, temos $h^{1} \smile h^{2}(c)=\left(h^{1} \otimes h^{2}\right)(\varphi(c))$.

A cohomologia de $Q(4 n)$ com coeficientes em $\mathbb{Z} / 2$ é dada por

$$
H^{i}(Q(4 n) ; \mathbb{Z} / 2)= \begin{cases}0, & \text { para } i=0 ; \\ \mathbb{Z} / 2 \oplus \mathbb{Z} / 2, \quad \text { gerado por } \beta \text { e } \beta^{\prime}, & \text { para } i=1 ; \\ \mathbb{Z} / 2 \oplus \mathbb{Z} / 2, \quad \text { gerado por } \gamma \text { e } \gamma^{\prime}, & \text { para } i=2 ; \\ \mathbb{Z} / 2, \quad \text { gerado por } \varepsilon, & \text { para } i=3 .\end{cases}
$$


onde $\beta, \beta^{\prime}, \gamma, \gamma^{\prime}$ e $\varepsilon$ são os cociclos básicos correspondentes às cadeias $b, b^{\prime}, c, c^{\prime}$ e $e$ geradoras da homologia de $Q(2 n)$ com coeficientes em $\mathbb{Z} / 2$.

Assim, a partir do que vimos acima e assumindo que o índice $n$ pode tomar os valores $2 m$ ou $2 m+1$, obtemos a seguinte tabela que descreve completamente a multiplicação no anel de cohomologia acima:

\begin{tabular}{|c|c|c|c|c|c|}
\hline$\smile$ & $\beta$ & $\beta^{\prime}$ & $\gamma$ & $\gamma^{\prime}$ & $\varepsilon$ \\
\hline$\beta$ & $m \gamma+\gamma^{\prime}$ & $\gamma^{\prime}$ & $\varepsilon$ & $\varepsilon$ & 0 \\
\hline$\beta^{\prime}$ & $\gamma^{\prime}$ & $\gamma$ & 0 & $\varepsilon$ & 0 \\
\hline$\gamma$ & $\varepsilon$ & 0 & 0 & 0 & 0 \\
\hline$\gamma^{\prime}$ & $\varepsilon$ & $\varepsilon$ & 0 & 0 & 0 \\
\hline$\varepsilon$ & 0 & 0 & 0 & 0 & 0 \\
\hline
\end{tabular}

Observação 6.1. A tabela acima fica completa se observarmos que $\left(\beta^{\prime}\right)^{3}=0$ e $\beta^{3}=(m+1) \varepsilon$.

Agora o caso dos coeficientes globais em $\mathbb{Z}$ :

$$
H^{i}(Q(4 n) ; \mathbb{Z})= \begin{cases}\mathbb{Z}, \quad \text { gerado por } \alpha & \text { para } i=0 ; \\ 0, & \text { para } i=1 ; \\ \mathbb{Z} / 2 \oplus \mathbb{Z} / 2, \quad \text { gerado por } \gamma \text { e } \gamma^{\prime}, & \text { para } i=2 ; \\ \mathbb{Z}, \quad \text { gerado por } \varepsilon, & \text { para } i=3 .\end{cases}
$$

onde $\alpha, \gamma, \gamma^{\prime}$ e $\varepsilon$ são os cociclos básicos correspondentes às cadeias $a, c, c^{\prime}$ e $e$ geradoras da homologia de $Q(2 n)$ com coeficientes em $\mathbb{Z}$.

Procedendo como no caso anterior, obtemos a seguinte tabela:

\begin{tabular}{|c|c|c|c|c|}
\hline$\smile$ & $\alpha$ & $\gamma$ & $\gamma^{\prime}$ & $\varepsilon$ \\
\hline$\alpha$ & $\alpha$ & $\gamma$ & $\gamma^{\prime}$ & $\varepsilon$ \\
\hline$\gamma$ & $\gamma$ & 0 & 0 & 0 \\
\hline$\gamma^{\prime}$ & $\gamma^{\prime}$ & 0 & 0 & 0 \\
\hline$\varepsilon$ & $\varepsilon$ & 0 & 0 & 0 \\
\hline
\end{tabular}


Observação 6.2. A tabela acima fica completa se observarmos que $\alpha^{n}=\alpha$.

A seguir o caso dos coeficientes locais obtidos a partir de uma representação $\rho_{x}: Q(2 n) \rightarrow \mathbb{Z} / 2$ tal que $\rho_{x}(x)=-1$ e $\rho_{x}(y)=1$. A notação segue o mesmo esquema apresentado nos dois casos anteriores.

$$
H^{i}\left(Q(4 n) ; \mathbb{Z}_{\rho_{x}}\right)=\left\{\begin{array}{lll}
0, & \text { para } i=0 ; \\
\mathbb{Z} / 2, & \text { gerado por } \beta, & \text { para } i=1 . \\
\mathbb{Z} / 2 & \text { gerado por } \gamma+\gamma^{\prime}, & \text { para } i=2 ; \\
\mathbb{Z} / 2 \text { gerado por } \varepsilon, & \text { para } i=3 .
\end{array}\right.
$$

Estrutura multiplicativa:

\begin{tabular}{|c|c|c|c|}
\hline$\smile$ & $\beta$ & $\gamma+\gamma^{\prime}$ & $\varepsilon$ \\
\hline$\beta$ & $\gamma+\gamma^{\prime}$ & 0 & 0 \\
\hline$\gamma+\gamma^{\prime}$ & 0 & 0 & 0 \\
\hline$\varepsilon$ & 0 & 0 & 0 \\
\hline
\end{tabular}

Observação 6.3. A tabela acima fica completa se observarmos que $\beta^{3}=0$.

Coeficientes locais tomados a partir da representação $\rho_{y}: Q(2 n) \rightarrow \mathbb{Z} / 2$ tal que $\rho_{y}(x)=1$ e $\rho_{y}(y)=-1:$

$$
H^{i}\left(Q(4 n) ; \mathbb{Z}_{\rho_{y}}\right)=\left\{\begin{array}{lll}
0, & \text { para } i=0 ; \\
\mathbb{Z}_{2}, & \text { gerado por } \beta^{\prime}, & \text { para } i=1 . \\
\mathbb{Z} / n & \text { gerado por } \gamma, & \text { para } i=2 ; \\
\mathbb{Z} / 2 & \text { gerado por } \varepsilon, & \text { para } i=3 .
\end{array}\right.
$$

Estrutura multiplicativa: 


\begin{tabular}{|c|c|c|c|}
\hline$\smile$ & $\beta^{\prime}$ & $\gamma$ & $\varepsilon$ \\
\hline$\beta^{\prime}$ & $\gamma$ & 0 & 0 \\
\hline$\gamma$ & 0 & 0 & 0 \\
\hline$\varepsilon$ & 0 & 0 & 0 \\
\hline
\end{tabular}

Observação 6.4. A tabela acima fica completa se observarmos que $\left(\beta^{\prime}\right)^{3}=0$.

Coeficientes locais tomados a partir da representação $\rho_{x, y}: Q(2 n) \rightarrow \mathbb{Z} / 2$ tal que $\rho_{x, y}(x)=-1$ e $\rho_{x, y}(y)=-1:$

$$
H^{i}\left(Q(4 n) ; \mathbb{Z}_{\rho_{x, y}}\right)=\left\{\begin{array}{lll}
0, & \text { para } i=0 ; \\
\mathbb{Z}_{2}, & \text { gerado por } \beta+\beta^{\prime}, & \text { para } i=1 . \\
\mathbb{Z} / 2 & \text { gerado por } \gamma^{\prime}, & \text { para } i=2 ; \\
\mathbb{Z} / 2 & \text { gerado por } \varepsilon, & \text { para } i=3 .
\end{array}\right.
$$

Estrutura multiplicativa:

\begin{tabular}{|c|c|c|c|}
\hline$\smile$ & $\beta+\beta^{\prime}$ & $\gamma^{\prime}$ & $\varepsilon$ \\
\hline$\beta+\beta^{\prime}$ & 0 & 0 & 0 \\
\hline$\gamma^{\prime}$ & 0 & 0 & 0 \\
\hline$\varepsilon$ & 0 & 0 & 0 \\
\hline
\end{tabular}

Observação 6.5. A tabela acima fica completa se observarmos que $\left(\beta+\beta^{\prime}\right)^{3}=0$.

\subsection{Os quadrados de Steenrod}

Nesta seção estudaremos brevemente homomorfismos relacionados à aplicação $\alpha \mapsto$ $\alpha^{2}$ que leva a classe de cohomologia $\alpha$ até o produto cup desta classe com ela mesma. A abordagem é a mesma usada em [6]. Estes homomorfismo são chamados de quadrados de Steenrod e são denotados por $S q^{i}: H^{n}\left(X ; \mathbb{Z}_{2}\right) \rightarrow H^{n+i}\left(X ; \mathbb{Z}_{2}\right)$. Ao contrário do produto cup, esta operação é estável, ou seja, invariante por suspensão. 
A maior parte das aplicações dos quadrados de Steenrod não dependem da forma como estes operadores são construídos mas sim de suas propriedades básicas - uma situação semelhante à da homologia comum, onde em geral os axiomas são suficientes para a maioria das aplicações. Por isto mostraremos apenas estas propriedades características e definições básicas, sem entrar em detalhes da sua construção.

Definição 6.2. Os quadrados de Steenrod $S q^{i}: H^{n}\left(X ; \mathbb{Z}_{2}\right) \rightarrow H^{n+i}\left(X ; \mathbb{Z}_{2}\right)$ são homomorfismos que satisfazem as seguintes propriedades:

1. $S q^{i}\left(f^{*}(\alpha)\right)=f^{*}\left(S q^{i}(\alpha)\right)$ para $f: X \rightarrow Y$.

2. $S q^{i}(\alpha+\beta)=S q^{i}(\alpha)+S q^{i}(\beta)$.

3. $S q^{i}(\alpha \smile \beta)=\sum_{j} S q^{j}(\alpha) \smile S q^{i-j}(\beta)$ (esta é a fórmula de Cartan).

4. $S q^{i}(\sigma(\alpha))=\sigma\left(S q^{i}(\alpha)\right)$, onde $\sigma: H^{n}\left(X ; \mathbb{Z}_{2}\right) \rightarrow H^{n+i}\left(\sum X ; \mathbb{Z}_{2}\right)$ é a suspensão dada pelo produto cross reduzido com um gerador de $H^{1}\left(S^{1} ; \mathbb{Z}_{2}\right)$.

5. $S q^{i}(\alpha)=\alpha^{2}$ se $i=|\alpha|$, e $S q^{i}(\alpha)=0$ quando $i>|\alpha|$.

6. $S q^{0}=11$, a identidade.

7. $S q^{1}$ é o $\mathbb{Z}_{2}$-bockstein $\beta$ associado com a seqüência de coeficientes $0 \rightarrow \mathbb{Z}_{2} \rightarrow$ $\mathbb{Z}_{4} \rightarrow \mathbb{Z}_{2} \rightarrow 0$.

Note que a primeira parte de (5) nos diz que os quadrados de Steenrod estendem a operação $\alpha \mapsto \alpha^{2}$ - que é um homomorfismo quando os coeficientes estão em $\mathbb{Z}_{2}$. A propriedade (4) diz que os quadrados de Steenrod são estáveis (ou seja, invariantes por suspensão) - lembre-se que a operação $\alpha \mapsto \alpha^{2}$ não tem esta propriedade.

\subsection{Relações entre fibrados vetoriais reais e complexos}

O objetivo desta seção surge do fato de que ao restringir os escalares de um fibrado vetorial complexo $\xi$ à $\mathbb{R}$, obtemos um fibrado vetorial real. Sempre que isso ocorre, temos um homomorfismo de grupos $\epsilon_{0}: K_{\mathbb{C}}(X) \rightarrow K_{\mathbb{R}}(X)$. Mas quando consideramos um fibrado vetorial real $\eta$ e tomamos seu produto tensorial com $\mathbb{C}$ temos um fibrado vetorial complexo $\eta \otimes \mathbb{C}$, chamado de complexificação de $\eta$. Assim, também temos um homomorfismo de anéis $\epsilon_{U}: K_{\mathbb{R}}(X) \rightarrow K_{\mathbb{C}}(X)$ tal que valem as relações $\epsilon_{0}\left(\epsilon_{U}(\eta)\right)=2 \eta$ e $\epsilon_{U}\left(\epsilon_{0}(\xi)\right)=\xi+\xi^{*}$, onde $\xi^{*}$ é o conjugado de $\xi$. Observe que para um fibrado vetorial real $\eta$, os fibrados complexos $\eta \otimes \mathbb{C}$ e $(\eta \otimes \mathbb{C})^{*}$ são isomorfos.

As demonstrações dos resultados aqui apresentados podem ser vistas em [9]. 
Teorema 6.1. Para um fibrado vetorial complexo $\xi$, vale a relação $c_{i}\left(\xi^{*}\right)=(-1)^{i} c_{i}(\xi)$.

Corolário 4. Se um fibrado complexo $\xi$ é isomorfo a $\xi^{*}$, então $2 c_{2 i+1}(\xi)=0$ para $0 \leq i$.

Definição 6.3. A i-ésima classe de Pontrjagin de um fibrado real $\xi$, denotada por $p_{i}(\xi)$ é $(-1)^{i} c_{2 i}(\xi \otimes \mathbb{C}) \in H^{4 i}(B, \mathbb{Z})$. Definimos também $p(\xi)=1+p_{1}(\xi)+\ldots \in$ $H^{*}(B, \mathbb{Z})$ como sendo a classe total de Pontrjagin.

Teorema 6.2. Seja $q: \mathbb{R} P^{2 n-1} \rightarrow \mathbb{C} P^{n-1}$ a aplicação que leva a reta determinada por $\{z,-z\}$ para $z \in S^{2 n-1}$ a reta complexa determinada por $z$. Então se $\xi$ é o fibrado por linhas real em $\mathbb{R} P^{2 n-1}$ e $\eta$ é o fibrado por linhas complexo em $\mathbb{C} P^{n-1}$, valem as seguintes afirmações:

- O fibrado $q^{*}(\eta)$ é isomorfo a $\epsilon_{U}(\eta)$;

- A classe $w_{2}\left(\epsilon_{0}(\eta)\right)$ é a redução módulo 2 de $c_{1}(\eta)$;

- $c_{1}(\eta)=e\left(\epsilon_{0}(\eta)\right)$.

Corolário 5. Sobre um espaço paracompacto, $w_{2}\left(\epsilon_{0}(\eta)\right)$ é a restrição de $c_{1}(\eta)$ módulo 2 para qualquer fibrado por linhas complexo $\eta$.

Corolário 6. Sobre um espaço paracompacto, $e\left(\epsilon_{0}(\eta)\right)$ é igual a $c_{1}(\eta)$ para qualquer fibrado por linhas complexo $\eta$.

Teorema 6.3. Um fibrado vetorial $\xi$ sobre um espaço $B$ é orientável se e só se $w_{1}(\xi)=0$

\subsection{Levantamentos de aplicações em torres de Postnikov}

Esta seção tem o objetivo de mostar um método alternativo para saber quais das aplicações encontradas nos três primeiros exemplos de 3.3 se levantam do segundo para o terceiro nível da torre de Postnikov, como indicado naquela seção. Recorremos ao cálculo das classes características dos fibrados sobre $O$. Para $G=S_{3}$ temos que a classe total trivial corresponde ao fibrado por linhas trivial sobre $O$, e então só podemos ter a classe total trivial para aqueles fibrados por 3-planos com orientação positiva cujo grupo estrutural é $\mathrm{SO}_{3}$, e de acordo com os resultados obtidos anteriormente, para este caso temos que apenas a classe da aplicação trivial se levanta de $\left[O,(B G)_{2}\right]$ para $\left[O,(B G)_{3}\right]$. 
No caso de $G=U_{2}$, sabemos que os 1-fibrados complexos sobre $O$ são classificados por $\left[O, B S^{1}\right]=H^{2}(O, \mathbb{Z})=\mathbb{Z}_{2} \oplus \mathbb{Z}_{2}$, então a idéia é verificarmos as classes de Chern dos 2-fibrados sobre $O$ obtidas a partir de somas de Whitney destes 1-fibrados. É claro que fibrados que não são equivalentes não tem as mesmas classes de Chern e a partir daí será possível classificar os 2-fibrados sobre $O$, como veremos a seguir.

Denotamos por $\lambda_{i}, i=1, \ldots, 4$ os elementos de $\left[O, B S^{1}\right]=\mathbb{Z}_{2} \oplus \mathbb{Z}_{2}$, e suas classes totais de Chern são dadas por

$$
\begin{gathered}
c\left(\lambda_{1}\right)=1+0 \\
c\left(\lambda_{2}\right)=1+a_{2} \\
c\left(\lambda_{3}\right)=1+a_{3} \\
c\left(\lambda_{4}\right)=1+a_{2}+a_{3},
\end{gathered}
$$

onde $a_{i} \in H^{2}(O, \mathbb{Z})$.

Queremos agora encontrar as classes totais de Chern para os fibrados obtidos através de somas de Whitney destes fibrados acima. Para isto faremos uso da propriedade $c\left(\lambda_{i} \oplus \lambda_{j}\right)=c\left(\lambda_{i}\right) \smile c\left(\lambda_{j}\right)$ onde $\smile$ indica o produto cup.

Observando que $1 \smile a_{i}=a_{i}$, os resultados obtidos são:

$$
\begin{gathered}
c\left(\lambda_{1} \oplus \lambda_{1}\right)=c\left(\lambda_{1}\right) \smile c\left(\lambda_{1}\right)=1 \smile 1 \\
c\left(\lambda_{1} \oplus \lambda_{2}\right)=c\left(\lambda_{1}\right) \smile c\left(\lambda_{2}\right)=1 \smile\left(1+a_{2}\right)=1 \smile 1+1 \smile a_{2},
\end{gathered}
$$

sendo que $1 \smile 1 \in H^{0}(X ; \mathbb{Z})$ e $1 \smile a_{2} \in H^{2}(X, \mathbb{Z})$.

$$
\begin{gathered}
c\left(\lambda_{1} \oplus \lambda_{3}\right)=c\left(\lambda_{1}\right) \smile c\left(\lambda_{3}\right)=1 \smile\left(1+a_{3}\right)=1 \smile 1+1 \smile a_{3} \\
c\left(\lambda_{1} \oplus \lambda_{4}\right)=c\left(\lambda_{1}\right) \smile c\left(\lambda_{4}\right)=1 \smile\left(1+a_{2}+a_{3}\right)=1 \smile 1+1 \smile a_{2}+1 \smile a_{3} \\
c\left(\lambda_{2} \oplus \lambda_{2}\right)=\left(1+a_{2}\right) \smile\left(1+a_{2}\right)=1 \smile 1+1 \smile a_{2}+a_{2} \smile 1+a_{2} \smile a_{2}=1+2\left(1 \smile a_{2}\right)+0=1
\end{gathered}
$$

Aqui usamos o fato de que $a_{2} \smile a_{2} \in H^{4}(O, \mathbb{Z}) \approx 0$ e também a propriedade do produto cup de que $a_{2} \smile 1=(-1)^{0 \cdot 2} 1 \smile a_{2}$, e ainda que como $1 \smile a_{2} \in H^{2}(O ; \mathbb{Z}) \approx$ $\mathbb{Z}_{2} \oplus \mathbb{Z}_{2}$ e então $1 \smile a_{2}+1 \smile a_{2}=0$.

Os resultados a seguir seguem de cálculos absolutamente análogos aos apresentados acima:

$$
\begin{gathered}
c\left(\lambda_{2} \oplus \lambda_{3}\right)=1 \smile 1+1 \smile a_{2}+1 \smile a_{3} \\
c\left(\lambda_{2} \oplus \lambda_{4}\right)=1 \smile 1+1 \smile a_{3} \\
c\left(\lambda_{3} \oplus \lambda_{3}\right)=1 \smile 1
\end{gathered}
$$




$$
\begin{gathered}
c\left(\lambda_{3} \oplus \lambda_{4}\right)=1 \smile 1+1 \smile a_{2} \\
c\left(\lambda_{4} \oplus \lambda_{4}\right)=1 \smile 1
\end{gathered}
$$

Note que obtivemos quatro classes de Chern distintas, e como existem exatamente quatro classes de aplicações de $O$ até $(B G)_{2}$, então concluímos que todas elas se levantam até $(B G)_{3}$.

Agora podemos tomar para cada elemento de $\left[O,(B G)_{2}\right]=\mathbb{Z}_{2} \oplus \mathbb{Z}_{2}$ uma aplicação $f: O \rightarrow(B G)_{3}$ na sua imagem inversa por $p_{n}$. O método introduzido por Larmore e exemplificado por nós aqui é válido para qualquer $f$ nestas condições e como $(B G)_{1}=$ *, pelo que foi mostrado aqui teremos que $f$ pertence sempre a uma mesma classe de homotopia, ou seja, a cada classe de homotopia em $\left[O,(B G)_{2}\right]$ corresponde uma única classe em $\left[O,(B G)_{3}\right]$. Como a recíproca é óbvia, temos que $\left[O,(B G)_{2}\right] \approx$ $\left[O,(B G)_{3}\right] \approx \mathbb{Z}_{2} \oplus \mathbb{Z}_{2}$, ou seja, temos exatamente quatro classes de fibrados sobre $O$ quando $G=U_{2}$.

\subsubsection{O caso de $\mathrm{O}_{3}$}

Temos que $\left[O,\left(B O_{3}\right)_{1}\right]=\left[O, K\left(\mathbb{Z}_{2}, 1\right)\right]=H^{1}\left(O, \mathbb{Z}_{2}\right)=\mathbb{Z}_{2} \oplus \mathbb{Z}_{2}$. Para sabermos quantos e quais destes elementos podem ser levantados até $\left(\mathrm{BO}_{3}\right)_{2}$ calcularemos as classes de Stiefel-Whitney das somas de Whitney de três 1-fibrados, sendo que estes últimos são classificados por $\left[O, B \mathbb{Z}_{2}\right]=\left[O, K\left(\mathbb{Z}_{2}, 1\right)\right]=H^{1}\left(O, \mathbb{Z}_{2}\right)=\mathbb{Z}_{2} \oplus \mathbb{Z}_{2}$, isto é, temos quatro fibrados $\lambda_{1}, \lambda_{2}, \lambda_{3}, \lambda_{4}$ com classes de Stiefel-Whitney totais dadas respectivamente por $1,1+a_{2}, \quad 1+a_{3}, \quad 1+a_{2}+a_{3}$, onde $a_{i} \in H^{1}\left(O, \mathbb{Z}_{2}\right)=\mathbb{Z}_{2} \oplus \mathbb{Z}_{2}$.

Aqui devemos tomar cuidado porque duas classes de fibrados com classes de Stiefel-Whitney diferentes podem ser levadas pela induzidada por $p_{n}$ até uma mesma classe de homotopia. Veja o diagrama abaixo, se $\left[f_{1}\right]$ e $\left[f_{2}\right]$ são duas classes distintas, podemos ter perfeitamente $\left[p_{n} f_{1}\right]=\left[p_{n} f_{2}\right]$ pois sabemos que $p_{n} f_{1}$ levanta até a mesma classe de $f_{1}$, mas nada aí garante que esta seja a mesma classe de $f_{2}$.

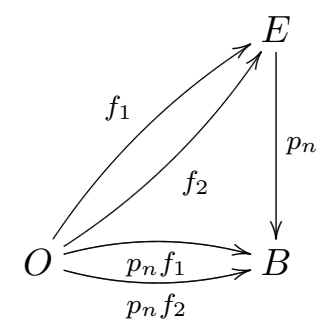

No nosso caso, calcular as classes de Stiefel-Whitney aqui só nos dá a quantidade de aplicações que levantam até $\left(\mathrm{BO}_{3}\right)_{2}$. A partir daí devemos ver quais são estas 
classes e aí sim aplicar o processo de Larmore para saber quantas classes temos na imagem inversa de cada $p_{n} f$. Observe que no caso de $G=U_{2}$ não foi preciso nos preocuparmos com isto, uma vez que já tínhamos calculado todas as possibilidades possíveis para as classes características totais dos 2-fibrados complexos sobre $O$, e estas eram apenas quatro, exatamente o número de classes de aplicações para as possíveis $p_{n} f$.

Aproveitando a notação estabelecida anteriormente, sejam $\lambda_{i}, \quad i=1, \ldots, 4$ os 3 -fibrados reais por linha sobre $O$, com classes totais $1,1+a_{2}, 1+a_{3}, 1+a_{2}+a_{3}$, respectivamente. Considere as seguintes somas de Whitney: $\lambda_{1} \oplus \lambda_{1} \oplus \lambda_{i}, \quad i=1, \ldots, 4$. Com cálculos análogos àqueles feitos anteriormente, temos que tais fibrados têm, como classe de Stiefel-Whitney total, respectivamente, $1,1+a_{2}, 1+a_{3}, 1+a_{2}+a_{3}$, o que significa que temos pelo menos quatro classes de homotopia de aplicações de $\mathrm{O}$ até $\left(\mathrm{BO}_{3}\right)_{2}$. Nosso trabalho agora será mostrar que a cada uma dessas classes de Stiefel-Whitney corresponde uma única classe de homotopia em $\left[O,\left(B O_{3}\right)_{1}\right]$, e portanto todas as quatro classes de $\left[O,\left(\mathrm{BO}_{3}\right)_{1}\right]$ se levantam..

Considere então o diagrama abaixo, onde $\gamma_{i}$ é o fibrado universal para $i=1,3$ :

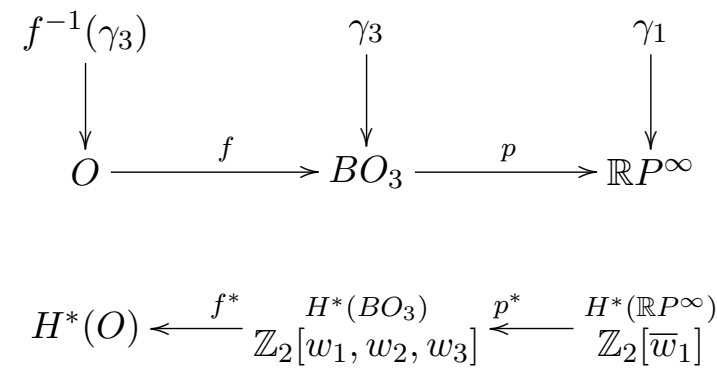

Note que $f^{-1}\left(\gamma_{3}\right)=(p f)^{-1}\left(\gamma_{1}\right)$. A classe total de $f^{-1}\left(\gamma_{3}\right)$ por um lado é dada por $f^{*}(w)=f^{*}\left(1+w_{1}+w_{2}+w_{3}\right)=1+f^{*}\left(w_{1}\right)+f^{*}\left(w_{2}\right)+f^{*}\left(w_{3}\right)$ e por outro lado também é dada por $(p f)^{*}\left(1+\bar{w}_{1}\right)=f^{*} p^{*}\left(1+\bar{w}_{1}\right)=f^{*}\left(1+p^{*}\left(\bar{w}_{1}\right)\right)$. Mas de acordo com [11], $p: B O_{n} \rightarrow \mathbb{R} P^{\infty}$ tem um levantamento $j$ de tal forma que $(p j)^{*}=\mathbb{1}^{*}$, e portanto $p^{*}$ é injetora. Logo, só podemos ter $p^{*}\left(\bar{w}_{1}\right)=w_{1}$, de onde $f^{*}(w)=1+f^{*}\left(w_{1}\right)$, ou seja, ao fibrado por linhas cuja classe total é $1+\bar{w}_{1}$ corresponde o 3 -fibrado sobre $O$ cuja classe total é $1+w_{1}$.

Trazendo isso para a nossa notação habitual, temos que ao fibrado por linhas sobre $O$ cuja classe total é $1+a_{2}$, por exemplo, corresponde o 3 -fibrado sobre $O$ cuja classe total é $1+a_{2}$, e assim sucessivamente. A partir daí com o que vimos anteriormente, temos que as quatro classes de $\left[O,\left(\mathrm{BO}_{3}\right)_{1}\right]$ se levantam até $\left[O,\left(B O_{3}\right)_{2}\right]$, agora só resta saber quantas classes de homotopia em $\left[\mathrm{O},\left(\mathrm{BO}_{3}\right)_{2}\right]$ temos para cada elemento em $\left[O,\left(\mathrm{BO}_{3}\right)_{1}\right]$. 


\section{Referências Bibliográficas}

[1] K. S. Brown. Cohomology of groups, Graduate texts in mathematics 8\%. Springer-Verlag, 1982.

[2] H. Cartan and S. Eilemberg. Homological algebra, Princeton mathematical series 19. Princeton University Press, 1st. edition, 2056.

[3] C. Chevalley. Theory of Lie groups. Princeton University Press, 1st. edition, 1946.

[4] J. F. Davis and P. Kirk. Lecture notes in Algebraic Topology. American Mathematical Society, 1st. edition, 2001.

[5] K. Gruenberg. Resolutions by relations. J. London Math. Soc., 35:481-494, 1960.

[6] A. Hatcher. Algebraic Topology. Cambridge University Press, 1st. edition, 2002.

[7] P. J. Hilton and S. Wylie. Homology theory: An introduction to algebraic topology,. Cambridge University Press, 1960.

[8] H. Hopf. Zum clifford-kleinschen raumproblem. Mathematische Annalen, 95:313-339, 1925.

[9] D. Husemoller. Fibre Bundles. McGraw-Hill Book Company, 1st. edition, 1966.

[10] L. L. Larmore. Map cohomology operations and enumeration of vector bundles. Journal of Mathematics and mechanics, 17, N. 2:199-208, 1967.

[11] L. L. Larmore. Twisted cohomology and enumeration of vector bundles. Pacific Journal of Math., 30,No 2:437-457, 1969.

[12] J. MacCleary. User's guide to spectral sequences, Mathematics Lecture Series, 12. Publish or Perish, Inc., 1st edition, 1985. 
[13] T. de Melo. Enumeração dos fibrados vetoriais sobre superfícies fechadas. ICMC/USP, 1 edition, 2005.

[14] J. Milnor and J. Stacheff. Lectures on characteristic classes / Notes by James Stasheff. Princeton University Press, 1 edition, 1957.

[15] M. Mimura and H. Toda. Topology of Lie Groups I and II. American Mathematical Society, 1st. edition, 1991.

[16] A. Minatta, R. Piccinini, and M. Spreafico. A note about the isotropy groups of 2-plane bundles over closed surfaces. Collect. Math., 54,No 3:283-291, 2003.

[17] R. A. Piccinini and M. Spreafico. Conjugacy classes in gauge groups. Queen's papers in pure and applied mathematics, 111, 1998.

[18] R. A. Piccinini and M. Spreafico. The milgram-steenrod construction of classifying spaces for topological groups. Exposition. Math., 16:97-130, 1998.

[19] W. R. Scott. Group Theory. Prentice-Hall Inc., 1st. edition, 1991.

[20] H. Seifert and W. Threlfall. A textbook of topology, translated by Michael A. Goldman. Academic Press, 1980.

[21] N. Steenrod. Topology of fibre bundles. Princeton University Press, 1951.

[22] P. Svengrowski and A. R. Shastri. Type of 3-manifolds and addition of relativistic kinks. Reviews in Math. Physics, 3 no 4:467-478, 1991.

[23] P. Svengrowski and S. Tomoda. Private communication.

[24] R. G. Swan. The theory of sheaves. The University of Chicago Press, 1st. edition, 1964.

[25] G. Whitehead. Elements of Homotopy Theory. Springer-Verlag, 1st. edition, 1978.

[26] J. A. Wolf. Spaces of constant curvature, McGraw-Hill series in higher mathematics. McGraw-Hill, 1967.

[27] Zassenhaus. Über endliche fastkörper. Abhandlungen aus dem Mathematischen Seminar der Hamburgischen Universität, 11:187-220, 1935. 